\title{
On finiteness of type IIB compactifications: magnetized branes on elliptic Calabi-Yau threefolds
}

\author{
Mirjam Cvetič ${ }^{a, b}$ James Halverson, ${ }^{c}$ Denis Klevers ${ }^{a}$ and Peng Song ${ }^{a}$ \\ ${ }^{a}$ Department of Physics and Astronomy, University of Pennsylvania, \\ Philadelphia, PA 19104-6396, U.S.A. \\ ${ }^{b}$ Center for Applied Mathematics and Theoretical Physics, University of Maribor, \\ Maribor, Slovenia \\ ${ }^{c}$ Kavli Institute for Theoretical Physics, University of California, \\ Santa Barbara, CA 93106-4030, U.S.A. \\ E-mail: cvetic@cvetic.hep.upenn.edu, jim@kitp.ucsb.edu, \\ klevers@sas.upenn.edu, songpeng@sas.upenn.edu
}

ABSTRACT: The string landscape satisfies interesting finiteness properties imposed by supersymmetry and string-theoretical consistency conditions. We study $\mathcal{N}=1$ supersymmetric compactifications of Type IIB string theory on smooth elliptically fibered Calabi-Yau threefolds at large volume with magnetized D9-branes and D5-branes. We prove that supersymmetry and tadpole cancellation conditions imply that there is a finite number of such configurations. In particular, we derive an explicitly computable bound on the number of magnetic flux quanta, as well as the number of D5-branes, which is independent of the continuous moduli of the setup. The proof applies if a number of easy to check geometric conditions of the twofold base are met. We show that these geometric conditions are satisfied for the almost Fano twofold bases given by each toric variety associated to a reflexive two-dimensional polytope as well as by the generic del Pezzo surfaces $d P_{n}$ with $n=0, \ldots, 8$. Physically, this finiteness proof shows that there exist a finite collection of four-dimensional gauge groups and chiral matter spectra in the 4D supergravity theories realized by these compactifications. As a by-product we explicitly construct all generators of the Kähler cones of $d P_{n}$ and work out their relation to representation theory.

KEYWORDS: D-branes, Superstring Vacua, Intersecting branes models

ARXiv EPRINT: 1403.4943 


\section{Contents}

1 Introduction 1

2 Magnetized branes on elliptically fibered Calabi-Yau manifolds $\quad 3$

2.1 Tadpole cancellation and SUSY conditions 4

2.2 Smooth elliptic Calabi-Yau threefolds 5

2.3 Basic geometry of almost Fano twofolds 6

$\begin{array}{ll}2.3 .1 \text { Hirzebruch surfaces } & 7\end{array}$

$\begin{array}{lll}\text { 2.3.2 Del Pezzo surfaces } & 7\end{array}$

2.3.3 Toric surfaces from reflexive polytopes 9

3 Finiteness of magnetized D9- \& D5-brane configurations 9

$\begin{array}{ll}3.1 \text { Prerequisites: definitions \& basic inequalities } & 11\end{array}$

$\begin{array}{lll}3.2 & \text { Warm up: finiteness for elliptic fibrations over } \mathbb{P}^{2} & 13\end{array}$

3.3 Proving finiteness for two-dimensional almost Fano bases 14

$\begin{array}{lll}\text { 3.3.1 Bounds on } \beta \text {-branes } & 15\end{array}$

$\begin{array}{lll}3.3 .2 & \text { Bounds on } n_{I}^{D 5} & 27\end{array}$

$\begin{array}{lll}\text { 3.3.3 } & \text { Bounds on } \gamma \text {-branes } & 29\end{array}$

4 Conclusions $\quad 30$

A Kähler cones of del Pezzo surfaces \& their $M_{\{i, k\}}$-matrices 31

B Geometric data of almost Fano twofolds for computing explicit bounds 37

C An analytic proof of positive semi-definiteness of the $M_{\{i, k\}}$-matrices $\quad 39$

\section{Introduction}

M-theory or superstring compactification to four dimensions remains the most promising framework for the unification of the fundamental forces in Nature. The set of associated low energy effective theories which can arise in consistent compactifications is known as the string landscape. There have been many efforts to quantify this space, with the hope of uncovering observable properties shared by large classes of vacua which lead to novel insights in particle physics or cosmology. However, this has proven to be a very difficult problem deserving a multi-faceted approach.

The traditional one is to study the effective scalar potential on moduli space and to examine its associated vacua; in general a variety of perturbative and non-perturbative effects are utilized to this end. For example, in the much studied moduli stabilization scenarios of Type IIB compactifications [1, 2], these effects include superpotential contributions from 
background Ramond-Ramond flux and D-instanton effects. Increasingly more detailed realizations of these constructions have been studied in recent years; for progress on vacua with explicit complex structure moduli stabilization, see [3, 4], and on constructing explicit de Sitter flux vacua with a chiral spectrum, see the recent [5]. While this progress is significant and provides excellent proofs of principle, a clear caveat to the explicit construction of vacua is the enormity of the landscape.

Another approach is to study properties of the landscape more broadly. In Type IIB flux compactifications this has included, for example, the importance of four-form fluxes in obtaining the observed value of the cosmological constant [6]; issues of computational complexity, including finding vacua in agreement with cosmological data [7] and the systematic computation of non-perturbative effective potentials [8]; and the distribution and number of various types of supersymmetric and non-supersymmetric vacua $[9,10]$. Progress has also been made in understanding vacua in strongly coupled corners of the landscape. For example there has been much progress in F-theory, beginning with [11, 12].

A final approach, which will be the one utilized in this paper, is to understand how consistency conditions and properties of the landscape differ from those of generic quantum field theories. The former case is motivated in part by the existence of a swampland [13] of consistent effective theories which do not admit a string embedding. There are a number of examples of limitations on gauge theories in the landscape not present in generic gauge theories. In weakly coupled theories with D-branes, Ramond-Ramond tadpole cancellation places stronger constraints [14-17] on low energy gauge theories than anomaly cancellation, which include additional anomaly nucleation constraints [18] on SU(2) gauge theories; see also [19] for a recent analysis of anomalies at strong coupling in F-theory; ranks of gauge groups are often bounded $[20,21]$; and the matter representations are limited by the fact that open strings have precisely two ends. While more matter representations are possible at strong coupling, the possibilities are still limited. For example, in F-theory the possible non-Abelian [22-30] and Abelian [31-39] matter representations are limited by the structure of holomorphic curves in the geometry.

In [40], Douglas and Taylor studied the landscape of intersecting brane models ${ }^{1}$ in Type IIA compactifications on a particular $\mathbb{Z}_{2} \times \mathbb{Z}_{2}$-orientifold. ${ }^{2}$ They found that the conditions on D6-branes necessary for $\mathcal{N}=1$ supersymmetry in four dimensions, together with the D6-brane tadpole cancellation condition required for consistency of the theory, allow only a finite number of such D6-brane configurations. ${ }^{3}$ In each configuration, the four-dimensional gauge group and matter spectrum can be determined explicitly. Thus, the finite number of D6-brane configurations gives a finite number of gauge sectors in a 4D supergravity theory that arise from these compactifications, and their statistics were studied explicitly. It is expected that the finiteness result which they obtained is a much more general consequence of supersymmetry and tadpole cancellation conditions, rather than a phenomenon specific to their construction. In fact, they proposed a potential generalization of their result to

\footnotetext{
${ }^{1}$ See [41-43] for reviews of these compactifications and their implications for particle physics.

${ }^{2}$ See $[21,44]$ for a finiteness proof of the number of supersymmetric D-branes for fixed complex structures of this orientifold and $[17,45]$ for a first construction of chiral $\mathcal{N}=1$ supersymmetric three-family models.

${ }^{3}$ See [46] for a counting of three family vacua, that yields eleven such vacua.
} 
theories with magnetized D9- and D5-branes on smooth elliptically fibered Calabi-Yau threefolds, which can also be motivated by mirror symmetry, for example.

In this paper, we demonstrate that finiteness results are indeed much more general phenomena, providing further evidence that the string landscape itself is finite. Specifically, in large volume Type IIB compactifications on many smooth elliptically fibered Calabi-Yau threefolds $\pi: X \rightarrow B$, we prove that there are finitely many configurations of magnetized D9- and D5-branes satisfying Ramond-Ramond tadpole cancellation and the conditions necessary for $\mathcal{N}=1$ supersymmetry in four dimensions. We formulate a general, mathematical proof showing the existence of computable, explicit bounds on the number of magnetic flux quanta on the D9-branes and on the number of D5-branes, which only depends on the topology of the manifold $B$ and is in particular independent of its Kähler moduli, as long as they are in the large volume regime of $X$. These bounds involve simple geometric quantities of the twofold base $B$ of $X$ and the proof applies to any base $B$ that satisfies certain geometric conditions, that are easy to check and summarized in this paper. Furthermore, we show that these conditions are met by the almost Fano twofold bases $B$ given by the toric varieties associated to all 16 reflexive two-dimensional polytopes and the generic del Pezzo surfaces $d P_{n}$ for $n=0, \ldots, 8$. This list in particular includes also the Hirzebruch surfaces $\mathbb{F}_{0}=\mathbb{P}^{1} \times \mathbb{P}^{1}, \mathbb{F}_{1}=d P_{1}$, and $\mathbb{F}_{2}$. In this work, we focus on the finiteness question only, leaving the analysis of gauge group and matter spectra for this finite set of configurations to future work.

This paper is organized is follows. In section 2 we provide the relevant background on Type IIB compactifications with magnetized D9- and D5-branes and elliptically fibered Calabi-Yau threefolds at large volume. We first discuss the tadpole and supersymmetry conditions of general such setups, then present a basic account on elliptically fibered CalabiYau threefolds and end with a detailed discussion of the geometries of the twofold bases $B=\mathbb{F}_{k}, d P_{n}$ and the 16 toric twofolds. In section 3 we prove the finiteness of such Dbrane configurations. We begin by rewriting the tadpole and supersymmetry constraints in a useful form for the proof and make some definitions, then show the power of these definitions by proving finiteness on $\mathbb{P}^{2}$. Finally, we prove the existence of explicit bounds on the number of fluxes and D5-branes, that apply certain geometric conditions on $B$ are satisfied. In section 4 we conclude and discuss possibilities for future work. In appendix A we discuss the detailed structure of the Kähler cone of generic del Pezzo surfaces $d P_{n}$ and give the proof of positive semi-definiteness of certain intersection matrices on these Kähler cones, which is essential for the proof. In appendix B we summarize the geometrical data of the considered almost Fano twofolds which is necessary to explicitly compute the bounds derived in this work.

While finishing this manuscript we learned about the related work [47] in which heterotic compactifications and their F-theory duals are constructed systematically.

\section{Magnetized branes on elliptically fibered Calabi-Yau manifolds}

We consider an $\mathcal{N}=1$ compactification of Type IIB string theory on a Calabi-Yau threefold $X$ with spacetime-filling D5-branes, magnetized D9-branes, i.e. D9-branes with magnetic 
fluxes, ${ }^{4}$ and an O9-plane. We group the D9-branes into stacks of $N^{\alpha}$ branes and their orientifold image branes. The corresponding line bundle magnetic fluxes in $H^{(1,1)}(X, \mathbb{Z})$ are denoted by $F^{\alpha}$, respectively, $-F^{\alpha}$ for the image brane. In addition, we add stacks of D5-branes wrapping a curve $\Sigma^{\mathrm{D} 5}$.

In the following discussion of these models ${ }^{5}$ we mainly follow the notations and conventions of [40], to which we also refer for more details. For a concise review see [42].

\subsection{Tadpole cancellation and SUSY conditions}

D-branes carry Ramond-Ramond charge and source flux lines that must be cancelled in the compact extra dimensions, in accord with Gauss' law. These give rise to the so-called tadpole cancellation conditions. The D5-brane tadpole cancellation conditions are

$$
n_{I}^{D 5}-T_{I}=\sum_{\alpha} N^{\alpha} \mathcal{K}\left(F^{\alpha}, F^{\alpha}, D_{I}\right), \quad \forall D_{I} \in H^{(1,1)}(X)
$$

(we note a sign difference between the D5-tadpoles ${ }^{6}$ in [40] and [42]; here, we use the sign in [42]) where $D_{I}$ is a basis of divisors on $X, \mathcal{K}(\cdot, \cdot, \cdot)$ is the classical triple intersection of three two-forms or their dual divisors, where we denote, by abuse of notation, a divisor $D_{I}$ and its Poincaré dual by the same symbol. Furthermore, we define the curvature terms

$$
T_{I}=\int_{D_{I}} c_{2}(X), \quad n_{I}^{\mathrm{D} 5}=\Sigma^{\mathrm{D} 5} \cdot D_{I},
$$

where $c_{2}(X)$ is the second Chern-class on $X$ and $\Sigma^{\mathrm{D} 5}$ is the curve wrapped by all D5-branes. The integral wrapping numbers $n_{I}^{\mathrm{D} 5}$ are positive if $\Sigma^{\mathrm{D} 5}$ is a holomorphic curve and the $D_{I}$ are effective divisors. Following [42], the D9-brane tadpole cancellation condition reads

$$
16=\sum_{\alpha} N^{\alpha}
$$

Compactification of Type IIB string theory on a Calabi-Yau manifold gives rise to a four-dimensional $\mathcal{N}=2$ supergravity theory. An O9-orientifold breaks half of these supersymmetries and give rise to an $\mathcal{N}=1$ supergravity theory at low energies. Only D9and D5-branes can be added in a supersymmetric way to this orientifold. However, this requires aligning the central charges $Z\left(F^{\alpha}\right)$ of the branes appropriately with the O9-plane. For consistency with the supergravity approximation, we have to assume that the Kähler parameters of the Calabi-Yau threefold $X$ are at large volume. In this case, the conditions on the central charges ${ }^{7}$ necessary for $\mathcal{N}=1$ supersymmetry, with $J$ denoting the Kähler form on $X$, reduce to

$$
3 \mathcal{K}\left(J, J, F^{\alpha}\right)=\mathcal{K}\left(F^{\alpha}, F^{\alpha}, F^{\alpha}\right), \quad \mathcal{K}(J, J, J)>3 \mathcal{K}\left(J, F^{\alpha}, F^{\alpha}\right)
$$

\footnotetext{
${ }^{4}$ For the generic case of gauge bundles with non-Abelian structure groups, see [48].

${ }^{5}$ These models were first proposed for model-building in [49].

${ }^{6}$ We thank Washington Taylor and Michael Douglas for helpful correspondence related to this issue.

${ }^{7}$ In general, the central charge (and also the Kähler potential on the Kähler moduli space) receives perturbative and non-perturbative $\alpha^{\prime}$ corrections. Recently it has been understood [50-53] that these corrections are captured by the so-called Gamma class $\hat{\Gamma}_{X}$ on $X$ rather than $\sqrt{T d_{X}}$. Since we study compactifications at large volume, these corrections can be neglected.
} 
to which we will refer in the following as the SUSY equality and the SUSY inequality respectively.

\subsection{Smooth elliptic Calabi-Yau threefolds}

We study compactifications where $X$ is a smooth elliptically fibered Calabi-Yau threefold over a complex two-dimensional base $B, \pi: X \rightarrow B$, with a single section $\sigma: B \rightarrow X$, the zero-section. The class of the section $\sigma$ is the base $B$. By the adjunction formula and the Calabi-Yau condition, the section $\sigma$ obeys the relation

$$
\sigma^{2}=-c_{1} \sigma
$$

where $c_{1}$ denotes the first Chern class of the base $B$. For a smooth threefold the second cohomology is given by $H^{(1,1)}(X)=\sigma H^{0}(B) \oplus \pi^{*} H^{(1,1)}(B)$. A basis of $H^{(1,1)}(X)$ generating the Kähler cone of $X$ is given by

$$
D_{I}=\left(D_{0}, D_{i}\right), \quad D_{0}=\sigma+\pi^{*} c_{1}, \quad I=0,1, \ldots, p \equiv h^{(1,1)}(B)
$$

with Poincaré duality implied when discussing divisors. The divisors $D_{i}, i=1, \ldots, p$, are inherited from generators of the Kähler cone of the base, by abuse of notation denoted by the same symbol as their counterparts in $B$. The divisor $D_{0}$ is dual to the elliptic fiber $\mathcal{E}$ in the sense that it does not intersect any curve in $B$, i.e. $D_{0} \cdot \sigma \cdot D_{i}=0$ by $(2.5)$, and obeys $D_{0} \cdot \mathcal{E}=1$. We note that $\mathcal{E}$ is an effective curve.

We emphasize that the requirement of a smooth elliptically fibered $X$, which means that the fibration can at most have $I_{1}$-fibers, restricts the choice of two-dimensional bases $B$. The bases we consider here are smooth almost Fano twofolds, which are the nine del Pezzo surfaces $d P_{n}, n=0, \ldots, 8$, that are the blow-ups of $\mathbb{P}^{2}$ at up to eight generic points, the Hirzebruch surfaces $\mathbb{F}_{k}, k=0,1,2$ and the toric surfaces described by the 16 reflexive two-dimensional polytopes. For these bases, the elliptic fibration $X$ is smooth.

We abbreviate the triple intersections of three divisors on $X$ as $\mathcal{K}_{I J K}=$ $\mathcal{K}\left(D_{I}, D_{J}, D_{K}\right)$. In the particular basis (2.6), we obtain the following structure of the triple intersections,

$$
\mathcal{K}_{i j k}=0, \quad \mathcal{K}_{00 i}=\sum_{j}^{p} b_{j} \mathcal{K}_{0 i j}, \quad \mathcal{K}_{000}=\sum_{i, j}^{p} b_{i} b_{j} \mathcal{K}_{0 i j}=\sum_{i}^{p} b_{i} \mathcal{K}_{00 i}
$$

where the first equation is a property of the fibration and the second and third relations can be derived using (2.5). We also introduce the $p \times p$-matrix

$$
(C)_{i j}:=\mathcal{K}\left(D_{0}, D_{i}, D_{j}\right)=\mathcal{K}_{0 i j}
$$

which defines a bilinear pairing on divisors on the base $B$. For the cases we consider here its signature is $(1, p-1)$ for $\mathbb{F}_{k}$ and $d P_{n}, n=1, \ldots, 8$, and $C=1$ for $\mathbb{P}^{2}=d P_{0}$. Note that it will be convenient at some places in this work to view $H^{(1,1)}(B)$ as a $p$-dimensional vector space equipped with an inner product (2.8). We denote the inner product of two 
vectors $v, w$ in $H^{(1,1)}(B)$ simply by $C(v, w)$. In addition, we view the first Chern class $c_{1}$ of $B$, the fluxes $F^{\alpha}$ and the Kähler form $J$ as column vectors

$$
j=\left(\begin{array}{c}
j_{1} \\
\cdot \\
\cdot \\
\cdot \\
j_{p}
\end{array}\right) \quad m^{\alpha}=\left(\begin{array}{c}
m_{1}^{\alpha} \\
\cdot \\
\cdot \\
\cdot \\
m_{p}^{\alpha}
\end{array}\right) \quad b=\left(\begin{array}{c}
b_{1} \\
\cdot \\
\cdot \\
\cdot \\
b_{p}
\end{array}\right)
$$

Here the components of these vectors are defined via the expansion w.r.t. the $D_{I}$ in (2.6),

$$
\pi^{*} c_{1}=\sum_{i=1}^{p} b_{i} D_{i}, \quad F^{\alpha}=m_{0}^{\alpha} D_{0}+\sum_{i=1}^{p} m_{i}^{\alpha} D_{i}, \quad J=j_{0} D_{0}+\sum_{i=1}^{p} j_{i} D_{i},
$$

where $b_{i} \in \mathbb{Q}^{+}, m_{I}^{\alpha} \in \mathbb{Q}$ and $j_{I} \in \mathbb{R}^{+} .8$

We emphasize that the flux quantization condition $F^{\alpha} \in H^{(1,1)}(X, \mathbb{Z})$ can be equivalently written as

$$
\int_{C} F^{\alpha} \in \mathbb{Z}, \quad \forall C \in H_{2}(X, \mathbb{Z}),
$$

where $C$ is any curve in $X$. Noting that the elliptic fiber $\mathcal{E}$ and the Kähler generators $D_{i}$ of $B$ are integral curves in $X$, this implies, using (2.10),

$$
\int_{\mathcal{E}} F^{\alpha}=m_{0}^{\alpha} \in \mathbb{Z}, \quad \int_{D_{i}} F^{\alpha}=\sum_{j}^{p} C_{i j} m_{j}^{\alpha} \in \mathbb{Z} .
$$

We conclude by noting that for smooth elliptically fibered Calabi-Yau threefolds, the second Chern class $c_{2}(X)$ can be computed explicitly, see e.g. [54] for a derivation. By adjunction one obtains $c_{2}(X)=12 \sigma \cdot c_{1}+\pi^{*}\left(c_{2}+11 c_{1}^{2}\right)$ with $c_{2}$ the second Chern class on $B$, employing the relation (2.5). Using this and (2.7) we evaluate the curvature terms in (2.2) as

$$
T_{0}=\int_{B}\left(c_{2}+11 c_{1}^{2}\right), \quad T_{i}=12 \int_{D_{i}} c_{1}=12 \mathcal{K}_{00 i},
$$

which is straightforward to evaluate for concrete bases $B$.

\subsection{Basic geometry of almost Fano twofolds}

In this section we briefly discuss the geometrical properties of the almost Fano twofolds $B=\mathbb{F}_{k}, d P_{n}$ and the toric surfaces. The discussion in this section is supplemented by the explicit computations of the Kähler cones of $d P_{n}$ in appendix A and the summary of the key geometric data of $\mathbb{F}_{k}, d P_{n}$ in appendix $\mathrm{B}$, which is critical for the proof in section 3.

\footnotetext{
${ }^{8}$ We allow here for rational coefficients $m_{I}^{\alpha}, b_{i}$ in the expansion of $F^{\alpha}, \pi^{*} c_{1}$ that are in the integral homology $H^{(1,1)}(X, \mathbb{Z})$ in order to account for the possibility of Kähler generators $D_{I}$ that only span a sublattice of $H^{(1,1)}(X, \mathbb{Z})$ of index greater than one. This can happen for non-simplicial Kähler cones.
} 


\subsubsection{Hirzebruch surfaces}

The Hirzebruch surfaces $\mathbb{F}_{k}$ are $\mathbb{P}^{1}$-bundles over $\mathbb{P}^{1}$ of the form $\mathbb{F}_{k}=\mathbb{P}(\mathcal{O} \oplus \mathcal{O}(k))$. There is an infinite family of such bundles for every positive $k \in \mathbb{Z}_{\geq 0}$.

The isolated section of this bundle, $S$, and the fiber $F$ are effective curves generating the Mori cone and spanning the entire second homology

$$
H_{2}\left(\mathbb{F}_{k}, \mathbb{Z}\right)=\langle S, F\rangle
$$

Their intersections read

$$
S^{2}=-k, \quad S \cdot F=1, \quad F^{2}=0 .
$$

From this we deduce that the generators $D_{i}, i=1,2$, of the Kähler cone, which are defined to be dual to the generators in (2.14), read

$$
D_{1}=F, \quad D_{2}=S+k F .
$$

The Chern classes on $\mathbb{F}_{k}$ read

$$
c_{1}\left(\mathbb{F}_{k}\right)=2 S+(2+k) F=(2-k) D_{1}+2 D_{2}, \quad c_{2}\left(\mathbb{F}_{k}\right)=4,
$$

which implies that the vector $b$ in $(2.9)$ is $b=(2-k, 2)^{T}$.

Using (2.15), we compute the triple intersections in (2.7), in particular (2.8), as

$$
C=\left(\begin{array}{cc}
0 & 1 \\
1 & k
\end{array}\right), \quad \mathcal{K}_{001}=2, \quad \mathcal{K}_{002}=2+k, \quad \mathcal{K}_{000}=8,
$$

from which the curvature terms in (2.13) immediately follow as

$$
T_{0}=92, \quad T_{1}=24, \quad T_{2}=24+12 k
$$

We emphasize that $\mathbb{F}_{k}$ by means of (2.17) is Fano for $k<2$ and almost Fano for $k=2$, since the coefficient $b_{1}=2-k \geq 0$. The general elliptic Calabi-Yau fibration $X$ over $F_{k}$ with $k=0,1,2$ is smooth and develops $I_{3}$-singularities for $k=3$ up to $I I^{*}$-singularities for $k=12$, before terminal singularities occur for $k>12$ [55]. Thus, we focus on the Hirzebruch surfaces with $k=0,1,2$.

\subsubsection{Del Pezzo surfaces}

The Fano del Pezzo surfaces $d P_{n}$ are the blow-up of $\mathbb{P}^{2}$ at up to eight generic points. ${ }^{9}$

Their second homology group is spanned by the pullback of the hyperplane on $\mathbb{P}^{2}$, denoted by $H$, and the classes of the exceptional divisors, denoted as $E_{i}, i=1, \ldots, n$,

$$
H_{2}\left(d P_{n}, \mathbb{Z}\right)=\left\langle H, E_{i=1, \ldots, n}\right\rangle .
$$

\footnotetext{
${ }^{9}$ See $[56,57]$ for recent computations of refined BPS invariants on del Pezzo surfaces as well as their interpretation in M-/F-theory.
} 
The intersections of these classes read

$$
H^{2}=1, \quad H \cdot E_{i}=0, \quad E_{i} \cdot E_{j}=-\delta_{i j} .
$$

The Chern classes on $d P_{n}$ read

$$
c_{1}\left(d P_{n}\right)=3 H-\sum_{i=1}^{n} E_{i}, \quad c_{2}\left(d P_{n}\right)=3+n .
$$

The Mori cone of $d P_{n}$ for $n>1$ is spanned by the curves $\Sigma$ obeying [58, 59]

$$
\Sigma^{2}=-1, \quad \Sigma \cdot\left[K_{d P_{n}}^{-1}\right]=1,
$$

where $\left[K_{d P_{n}}^{-1}\right]$ is the anti-canonical divisor in $d P_{n}$, which is dual to $c_{1}\left(d P_{n}\right)$. By adjunction, we see that the curves obeying (2.23) obey the necessary condition for being $\mathbb{P}^{1}$ 's. By solving the conditions (2.23) with the ansatz $a_{0} H+\sum_{i=1}^{n} a_{i} E_{i}$ for $a_{0}, a_{i} \in \mathbb{Z}$, we obtain a cone that is simplicial, i.e. generated by $h^{(1,1,)}(B)=1+n$ generators, for $n=0,1,2$ and non-simplicial for $n>2$. The number of generators, beginning with $d P_{2}$, furnish irreducible representations of $A_{1}, A_{1} \times A_{2}, A_{4}, D_{5}, E_{n}$, for $n=6,7,8$, which concretely are $\mathbf{3}, \mathbf{2} \otimes \mathbf{3}$, 10, 16, 27, 56, 248. ${ }^{10}$ For the simplicial cases the Mori cone reads

$$
\mathbb{P}^{2}:\langle H\rangle, \quad d P_{1}:\left\langle E_{1}, H-E_{1}\right\rangle, \quad d P_{2}:\left\langle E_{1}, E_{2}, H-E_{1}-E_{2}\right\rangle
$$

and we refer to appendix A for more details on the non-simplicial cases.

Consequently, also the Kähler cones of the $d P_{n}$, which are the dual of the Mori cones defined by (2.23), are non-simplicial for $n>2$. The Kähler cone is spanned by rational curves $\Sigma$ obeying

$$
\Sigma^{2}=0, \quad \Sigma \cdot\left[K_{d P_{n}}^{-1}\right]=2 \quad \text { or } \quad \Sigma^{2}=1, \quad \Sigma \cdot\left[K_{d P_{n}}^{-1}\right]=3,
$$

which again implies by adjunction that $\Sigma=\mathbb{P}^{1}$. The solutions over the integers of these conditions yield the generators of the Kähler cone of $d P_{n}$ which again follow the representation theory of the above mentioned Lie algebras. The number of generators, starting with $d P_{0}$, is $1,2,3,5,10,26,99,702$ and 19440, see appendix A. In the simplicial cases, the Kähler cone generators read

$$
\mathbb{P}^{2}: D_{1}=H, \quad d P_{1}: D_{1}=H-E_{1}, D_{2}=H, \quad d P_{2}: D_{1}=H-E_{1}, D_{2}=H-E_{2}, D_{3}=H
$$

Generically, for $n \geq 2$ the vector $c_{1}\left(d P_{n}\right)$ is the center both of the Kähler and Mori cone. This implies that for all del Pezzo surfaces, the coefficients $b_{i}$ are positive. For the simplicial Kähler cones, this can be computed explicitly. For the non-simplicial cases we will argue in appendix $\mathrm{A}$, that a covering of the Kähler cone by simplicial subcones, i.e. subcones with $h^{(1,1)}$ generators, with all $b_{i} \geq 0$ always exists. We note that for all $d P_{n}$, the defining property of the Kähler cone (2.25), together with (2.7), implies the intersections

$$
\mathcal{K}_{00 i}=2,3, \quad \mathcal{K}_{000}=9-n .
$$

\footnotetext{
${ }^{10}$ The genuine roots in $H_{2}\left(d P_{n}\right)$ are the -2 -curves orthogonal to $\left[K_{d P_{n}}^{-1}\right]$, i.e. $\alpha_{i}=E_{i}-E_{i+1}, i=$ $1, \ldots, n-1, \alpha_{n}=H-E_{1}-E_{2}-E_{3}$ for $n>2$. These act on $H_{2}\left(d P_{n}\right)$ by means of the Weyl group, cf. [58].
} 
In addition, by explicit computations we check in general that all $C_{i j} \geq 0$ for all pairs of Kähler cone generators. The intersections (2.27) together with (2.21), (2.22) further imply that the curvature terms in (2.13) read

$$
T_{0}=102-10 n, \quad T_{i}=24,36
$$

For the three simplicial cases of $\mathbb{P}^{2}, d P_{1}$ and $d P_{2}$, we compute the matrices $(2.8)$ in the basis (2.26) as

$$
C_{\mathbb{P}^{2}}=1, \quad C_{d P_{1}}=\left(\begin{array}{ll}
0 & 1 \\
1 & 1
\end{array}\right), \quad C_{d P_{2}}=\left(\begin{array}{lll}
0 & 1 & 1 \\
1 & 0 & 1 \\
1 & 1 & 1
\end{array}\right) .
$$

We emphasize that the del Pezzos $d P_{n}$ by means of (2.27) are Fano for $n<9$ and almost Fano for $n=9$, since $c_{1}^{2}=0$. The surface $d P_{9}$ is the rational elliptic surface. Its Mori cone is the Mordell-Weil group of rational sections by (2.23). Thus, it as well as its dual Kähler cone is infinite dimensional. We will only consider the Fano del Pezzo surfaces $d P_{n}, n<9$.

\subsubsection{Toric surfaces from reflexive polytopes}

Toric surfaces obtained from fine star triangulations of reflexive polytopes are smooth almost Fano twofolds. ${ }^{11}$ There are 16 such polytopes in two dimensions, which are displayed in figure 1.

A number of these twofolds are simply toric descriptions of previously described surfaces. Specifically, these are $\mathbb{P}^{2}, d P_{1}, d P_{2}, d P_{3}, \mathbb{F}_{0}$ and $\mathbb{F}_{2}$ which are described by polytopes 1, 3, 5, 7, 2 and 4, respectively. From the form of some of the other polytopes it is clear that they can be obtained from $\mathbb{P}^{2}, d P_{1}, d P_{2}$, or $d P_{3}$ via toric blow-up. For example, reflecting polytope 7 through the vertical axis going through its center and performing a toric blow-up associated to the point $(-1,1)$, one obtains polytope 12. Thus, the smooth Fano surface associated to polytope 12 is a toric realization of $d P_{4}$ at a non-generic point in its complex structure moduli space.

The toric varieties associated to all these 16 reflexive polytopes can be constructed explicitly using the software package Sage [61]. The intersections (2.7), (2.8) are readily constructed in a given fine star triangulation and the Kähler cone can be obtained. We summarize the geometric data necessary for the computation of the bounds derived below in the proof in appendix B.

\section{Finiteness of magnetized D9- \& D5-brane configurations}

In this section we bound the number of possible gauge sectors arising in the considered compactifications of Type IIB string theory.

\footnotetext{
${ }^{11}$ See the recent [60] for a systematic study of the quantum geometry of the elliptically fibered Calabi-Yau manifolds over these bases.
} 


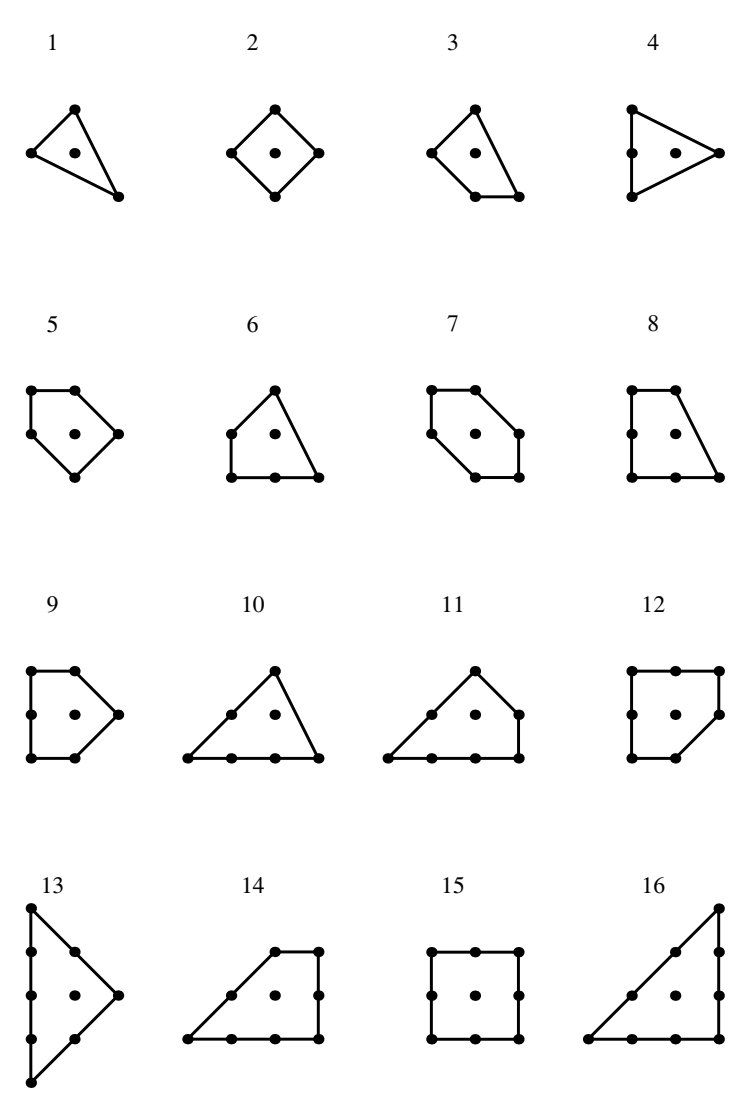

Figure 1. The sixteen two-dimensional reflexive polytopes which define the almost Fano toric surfaces via their fine star triangulations.

As emphasized in section 2, the number $N^{\alpha}$ of branes in a stack and their associated magnetic fluxes $F^{\alpha}$ are subject to the consistency conditions imposed by tadpole cancellation conditions (2.1), (2.3) and the SUSY conditions (2.4). Since the numbers $N^{\alpha}$ of D9-branes are bounded by (2.3), it is therefore the goal of this proof to bound the flux quanta $F^{\alpha}$ and the number of D5-branes in $\Sigma^{\mathrm{D} 5}$.

Most of the proofs in this work have the same basic structure. The key point is to find a bound on the number of different flux configurations $F^{\alpha}$ and D5-branes $\Sigma^{\mathrm{D} 5}$ at an arbitrary point in the large volume regions of Kähler moduli space, i.e. a bound that is independent of the Kähler moduli. As we will see, proving this requires an intriguing interplay between both the tadpole conditions (2.1), (2.3) and the SUSY conditions (2.4), a general rubic which was also used in the proof of [40]. ${ }^{12}$ In addition, the following proof applies if a list of geometrical properties, listed at the beginning of section 3.3, are satisfied. These are obeyed for the considered examples $B=\mathbb{F}_{k}, d P_{n}$ and the toric surfaces.

Before delving into the details of the proof, let us introduce a very important notation. Because of their fundamentally different contributions to (2.1), (2.3) and (2.4) it is useful

\footnotetext{
${ }^{12}$ The interplay between SUSY and tadpole conditions has also been used in $[21,44]$ for rigid $\mathbb{Z}_{2} \times \mathbb{Z}_{2^{-}}$ orientifolds and for other models in $[62,63]$.
} 
to split D9-brane stacks into to qualitatively different types according to their flux quanta. We denote D9-brane stacks with $m_{0}^{\alpha} \neq 0$ as $\beta$-branes, and those with $m_{0}^{\alpha}=0$ as $\gamma$-branes:

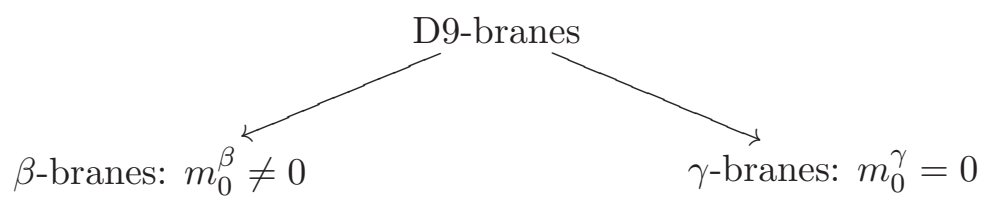

In addition, in the rest of this section we label fluxes of a $\beta$ - and $\gamma$-brane by $m_{I}^{\beta}$ and $m_{I}^{\gamma}$, respectively.

We begin in section 3.1 by preparing for the general finiteness proof by writing out the tadpoles and SUSY conditions of section 2 for elliptically fibered Calabi-Yau threefolds $X$. We also make certain definitions and deduce a number of simple inequalities and bounds, that will be essential for the later discussion. Then, in section 3.2 we prove finiteness for the special base $B=\mathbb{P}^{2}$, which will demonstrate the usefulness of the definitions of the previous section and serve as a warm-up for the general proof in section 3.3.

\subsection{Prerequisites: definitions \& basic inequalities}

In this section we make some general definitions and observations necessary to formulate and organize the proof in section 3.3.

As a starting point, we observe that the SUSY conditions (2.4) must be satisfied by each brane stack, but only involve the direction along the Kähler class $j$, whereas the tadpole conditions (2.1) have to be obeyed for each divisor $D_{I}$, but are summed across brane stacks. Thus, in order to bound each component $m_{I}^{\alpha}$ of every flux vector $m^{\alpha}$, labelled by the brane stack $\alpha$, it is crucial to identify quantities, that enter both types of constraints, when rewritten in a particular form.

To this end, we write out the tadpole conditions explicitly in the basis of divisors (2.6). The conditions (2.1) for $I=0$, to which we will refer in the future as the $0^{\text {th }}$-tadpole, reads

$$
0^{\text {th }} \text {-tadpole : } n_{0}^{D 5}-T_{0}=\underbrace{\sum_{\beta} N^{\beta} C\left(b+\frac{m^{\beta}}{m_{0}^{\beta}}, b+\frac{m^{\beta}}{m_{0}^{\beta}}\right)\left(m_{0}^{\beta}\right)^{2}}_{\beta \text { brane contributions }}+\underbrace{\sum_{\gamma} N^{\gamma} C\left(m^{\gamma}, m^{\gamma}\right)}_{\gamma \text { brane contributions }}
$$

where we used (2.8) and (2.9) and emphasized the respective contributions from $\beta$-branes and $\gamma$-branes. For $I=i$, to which we will refer as the $i^{\text {th }}$-tadpole, the tadpole $(2.1)$ reads

$$
i^{\text {th }} \text {-tadpole : } n_{i}^{D 5}-T_{i}=\underbrace{\sum_{\beta} N^{\beta} t_{i}^{\beta}\left(m_{0}^{\beta}\right)^{2}}_{\beta \text { brane contributions }} \quad \text { with } \quad t_{i}^{\beta} \equiv 2 \sum_{j=1}^{p} C_{i j}\left(\frac{b_{j}}{2}+\frac{m_{j}^{\beta}}{m_{0}^{\beta}}\right) .
$$

We note that the first term in $t_{k}^{\beta}$ can be written as $\sum_{j} b_{j} C_{i j}=\mathcal{K}_{00 i}$ which is an integer by (2.18), (2.27) and table 5 . The quantities $t_{i}^{\beta}$ can be defined for $\beta$-branes and play an important in the proof, because they naturally appear in the SUSY constraints. We 
emphasize that while both $\beta$-branes and $\gamma$-branes contribute to the $0^{\text {th }}$-tadpole condition, only $\beta$-branes contribute to the $i^{\text {th }}$-tadpole as is indicated by the braces in (3.2), (3.3).

We note that one can immediately deduce a lower bound on the left hand side of (3.2) and (3.3) by setting the positive numbers $n_{I}^{\mathrm{D} 5}=0$ :

$$
-T_{0} \leq \sum_{\beta} N^{\beta} C\left(b+\frac{m^{\beta}}{m_{0}^{\beta}}, b+\frac{m^{\beta}}{m_{0}^{\beta}}\right)\left(m_{0}^{\beta}\right)^{2}+\sum_{\gamma} N^{\gamma} C\left(m^{\gamma}, m^{\gamma}\right), \quad-T_{i} \leq \sum_{\beta} N^{\beta} t_{i}^{\beta}\left(m_{0}^{\beta}\right)^{2} .
$$

These lower bounds on the $i^{\text {th }}$-tadpoles imply, as we will see, that if the $t_{i}^{\beta}$ are bounded above, then they are automatically bounded below. This can be seen by bringing the bounded positive contribution to the left hand side of (3.4).

For $\beta$-branes, which have $m_{0}^{\beta} \neq 0$, it useful to divide the SUSY equality (2.4) by $m_{0}^{\beta}$. Using again (2.8) and (2.9), we write the first condition in (2.4) to obtain

$$
\left[3 C\left(\frac{j}{j_{0}}, \frac{j}{j_{0}}\right)+6 C\left(\frac{b}{2}+\frac{j}{j_{0}}, b+\frac{m^{\beta}}{m_{0}^{\beta}}\right)\right] j_{0}^{2}=\left[\frac{1}{4} \mathcal{K}_{000}+3 C\left(\frac{b}{2}+\frac{m^{\beta}}{m_{0}^{\beta}}, \frac{b}{2}+\frac{m^{\beta}}{m_{0}^{\beta}}\right)\right]\left(m_{0}^{\beta}\right)^{2} .
$$

The SUSY inequality in (2.4) for $\beta$-branes can be combined with the SUSY equality (3.5) as follows. By dividing the SUSY inequality in (2.4) by $j_{0}$ and subtracting the SUSY equality (3.5), we obtain after a few lines of algebra the following inequality:

$$
0>\frac{1}{2} \mathcal{K}_{000}+6 C\left(\frac{b}{2}+\frac{m^{\beta}}{m_{0}^{\beta}}, \frac{b}{2}+\frac{j}{j_{0}}\right) .
$$

This can equivalently be written in the form

$$
0>\frac{1}{2} \mathcal{K}_{000}+3 \sum_{i}^{p} t_{i}^{\beta}\left(\frac{b_{i}}{2}+\frac{j_{i}}{j_{0}}\right)
$$

and we see that the expression $t_{i}^{\beta}$, which explicitly appears in the $i^{\text {th }}$-tadpole conditions in (2.13), appears also in this manipulation of the SUSY constraints.

We note that (3.7) can be related to the tadpole conditions. By multiplying (3.7) by $N^{\beta}\left(m_{0}^{\beta}\right)^{2}$ and summing over $\beta$, we employ the right hand side of (3.3) to obtain

$0>\frac{1}{2} \mathcal{K}_{000} \sum_{\beta} N^{\beta}\left(m_{0}^{\beta}\right)^{2}+3 \sum_{i=1}^{p}\left(n_{i}^{\mathrm{D} 5}-T_{i}\right)\left(\frac{b_{i}}{2}+\frac{j_{i}}{j_{0}}\right) \geq \frac{1}{2} \mathcal{K}_{000} \sum_{\beta} N^{\beta}\left(m_{0}^{\beta}\right)^{2}-3 \sum_{i=1}^{p} T_{i}\left(\frac{b_{i}}{2}+\frac{j_{i}}{j_{0}}\right)$,

where we set $n_{i}^{\mathrm{D} 5}=0$ in the last inequality. This condition is used throughout the proof.

Next, we demonstrate that it is possible to also rewrite the SUSY equality (3.5) and the $0^{\text {th }}$-tadpole (3.2) in a form that manifestly contains the quantities $t_{i}^{\beta}$. To this end, we first define for each distinct pair of indices $\{i, k\}, i \neq k$, the matrix $M_{\{i, k\}}$ whose $(j, l)$-th entry in the basis $D_{i}$ is:

$$
\left(M_{\{i, k\}}\right)_{j l}=x_{\{i, k\}} C_{i j} C_{k l}+x_{\{i, k\}} C_{i l} C_{k j}-C_{j l}
$$


where $x_{\{i, k\}} \in \mathbb{Q}^{+}$is a non-negative rational number. This number has to be chosen such that its corresponding $M_{\{i, k\}}$ is positive semi-definite. We note, that the matrices $M_{\{i, k\}}$ resemble the stress energy tensor of a system of free particles, cf. appendix C. We use this to show that, if the first condition in section 3.3 is met, there always exists an $x_{\{i, k\}}$ so that these matrices are positive semi-definite, see appendices A and C. Thus, throughout the rest of this proof we assume that all matrices $M_{\{i, k\}}$ are positive semi-definite.

With this definition, the SUSY equality (3.5) and $0^{\text {th }}$-tadpole (3.2) can be written as

$$
\left[3 C\left(\frac{j}{j_{0}}, \frac{j}{j_{0}}\right)+6 C\left(\frac{b}{2}+\frac{j}{j_{0}}, b+\frac{m^{\beta}}{m_{0}^{\beta}}\right)\right] j_{0}^{2}=\left[\frac{1}{4} \mathcal{K}_{000}+\frac{3}{2} x_{\{i, k\}} t_{i}^{\beta} t_{k}^{\beta}-3 M_{\{i, k\}}\left(\frac{b}{2}+\frac{m^{\beta}}{m_{0}^{\beta}}, \frac{b}{2}+\frac{m^{\beta}}{m_{0}^{\beta}}\right)\right]\left(m_{0}^{\beta}\right)^{2}
$$

and

$$
n_{0}^{D 5}-T_{0}=\underbrace{\sum_{\beta} N^{\beta}\left[\frac{1}{2} x_{\{i, k\}} \tilde{t}_{i}^{\beta} \tilde{t}_{k}^{\beta}-M_{\{i, k\}}\left(b+\frac{m^{\beta}}{m_{0}^{\beta}}, b+\frac{m^{\beta}}{m_{0}^{\beta}}\right)\right]\left(m_{0}^{\beta}\right)^{2}}_{\beta-\text { brane contributions }}+\underbrace{\sum_{\gamma} N^{\gamma} C\left(m^{\gamma}, m^{\gamma}\right)}_{\gamma-\text { brane contributions }},
$$

respectively, where we indicated the contributions from $\beta$ - and $\gamma$-branes by braces and used the short hand notation

$$
\tilde{t}_{i}^{\beta}=\mathcal{K}_{00 i}+t_{i}^{\beta}
$$

As we will see, the proof of section 3.3 applies whenever the $M$-matrices in (3.9) are all positive semi-definite. In fact, for all the bases $B$ of the threefold $X$ considered, this matrix is positive semi-definite. For $\mathbb{P}^{2}, \mathbb{P}^{1} \times \mathbb{P}^{1}, d P_{1}, d P_{2}$, and $\mathbb{F}_{2}$ the $M$-matrix can be readily computed in the Kähler cone basis, and indeed, it is positive semi-definite. However, for $d P_{n}$ with $n \geq 3$ there exists a significant complication since in these examples, the Kähler cone is non-simplicial, as mentioned in section 2. In these cases, we cover the Kähler cone by simplicial subcones consisting of $h^{(1,1)}$ generators and compute the $M$-matrix (3.9) for this choice. As demonstrated in appendix A, for $d P_{n}, n<9$, the $M$-matrices are positive semi-definite for all such subcones. For the toric surfaces, we refer to appendix B for positive semi-definiteness of the matrices (3.9). Thus, for the rest of the paper we can assume that all $M_{\{i, k\}}$ are positive semi-definite for these bases.

\subsection{Warm up: finiteness for elliptic fibrations over $\mathbb{P}^{2}$}

Before proceeding on to more difficult examples, let us prove finiteness in the simplest example of $B=\mathbb{P}^{2}$. In particular, in this example we will demonstrate the usefulness of the derived inequality (3.6) and (3.8).

For an elliptically fibered Calabi-Yau threefold $X$ over $B=\mathbb{P}^{2}$, the relevant geometrical data following from $(2.22),(2.27),(2.28)$ and $(2.29)$ is:

$$
\mathcal{K}_{000}=9, \quad \mathcal{K}_{001}=3, \quad \mathcal{K}_{011} \equiv C_{11}=1, \quad b_{1}=3, \quad T_{1}=36 .
$$

Using this the inequality (3.6) reduces to

$$
0>\mathcal{K}_{001}\left(m_{0}^{\beta}\right)^{2}+2 \mathcal{K}_{011} m_{0}^{\beta} m_{1}^{\beta} .
$$


The tadpole for $D_{1}$ reads

$$
n_{1}^{D 5}-T_{1}=\sum_{\beta} N^{\beta}\left[\mathcal{K}_{001}\left(m_{0}^{\beta}\right)^{2}+2 \mathcal{K}_{011} m_{0}^{\beta} m_{1}^{\beta}\right] .
$$

By (3.13), the right hand side of (3.14) must be negative. Thus we have a bound for $n_{1}^{\mathrm{D} 5}$, given by

$$
n_{1}^{\mathrm{D} 5}<T_{1} .
$$

In addition, for each $\beta$-brane we deduce from (3.13) that

$$
\begin{aligned}
0 & <\left|m_{0}^{\beta}\right|\left|\mathcal{K}_{001} m_{0}^{\beta}+2 \mathcal{K}_{011} m_{1}^{\beta}\right|=\left|\mathcal{K}_{001}\left(m_{0}^{\beta}\right)^{2}+2 \mathcal{K}_{011} m_{0}^{\beta} m_{1}^{\beta}\right| \\
& \leq \sum_{\beta} N^{\beta}\left|\mathcal{K}_{001}\left(m_{0}^{\beta}\right)^{2}+2 \mathcal{K}_{011} m_{0}^{\beta} m_{1}^{\beta}\right| \leq T_{1}
\end{aligned}
$$

Notice that $\left|\mathcal{K}_{001} m_{0}^{\beta}+2 \mathcal{K}_{011} m_{1}^{\beta}\right|$ is a non-zero integer by virtue of the strict inequality (3.13). This implies the bound

$$
\left|m_{0}^{\beta}\right| \leq T_{1} .
$$

Next, since $\left|\mathcal{K}_{001} m_{0}^{\beta}+2 \mathcal{K}_{011} m_{1}^{\beta}\right| \leq T_{1} /\left|m_{0}^{\beta}\right|$ and $\left|m_{0}^{\beta}\right|$ is bounded, $m_{1}^{\beta}$ is also bounded as

$$
\left|m_{1}^{\beta}\right| \leq \frac{1}{2 \mathcal{K}_{011}}\left(\frac{T_{1}}{\left|m_{0}^{\beta}\right|}+\mathcal{K}_{001}\left|m_{0}^{\beta}\right|\right) .
$$

Thus we have shown that the magnetic flux quanta $m^{\beta}$ associated to $\beta$-branes are bounded.

A bound on the flux quanta of $\gamma$-branes is straightforward to obtain. The SUSY equality in (2.4) for each $\gamma$-brane is $\mathcal{K}_{011}\left(\frac{b_{1}}{2}+\frac{j_{1}}{j_{0}}\right) m_{1}^{\gamma}=0$. Since $\mathcal{K}_{011} \neq 0$ and $\left(\frac{b_{1}}{2}+\frac{j_{1}}{j_{0}}\right)$ is strictly positive, we must have $m_{1}^{\gamma}=0$. Since a $\gamma$-brane by definition has $m_{0}^{\gamma}=0$, the flux quanta of $\gamma$-branes are trivially bounded. This completes the proof for $B=\mathbb{P}^{2}$.

\subsection{Proving finiteness for two-dimensional almost Fano bases}

In this section we present the general proof of the finiteness of the number of consistent Type IIB compactification with magnetized D9-branes on smooth elliptically fibered CalabiYau threefolds. As discussed before the bases $B$ for which the presented proof has been developed are the two-dimensional almost Fano varieties. These are the del Pezzo surfaces $d P_{n}, n=0, \ldots, 8$, with the case of $d P_{0}=\mathbb{P}^{2}$ discussed in the previous section 3.2 , the Hirzebruch surfaces $\mathbb{F}_{k}, k=0,1$, including the almost Fano $\mathbb{F}_{2}$, as well as the toric surfaces.

The geometrical properties that are essential for the following proof are the smoothness of the generic elliptic Calabi-Yau fibration over them, as well as the following list of properties:

(1) all Kähler cone generators of $B$ are time- or light-like vectors in the same light-cone.

(2) positivity of the coefficients $b_{i}$ in (2.10), i.e. $b_{i} \geq 0$ for all $i$.

(3) positivity and integrality of $\mathcal{K}_{00 i}$ as defined in (2.7), i.e. $\mathcal{K}_{00 i} \in \mathbb{Z}_{\geq 0}$ for all $i$. 
(4) the signature of the matrix $C_{i j}$ defined in $(2.8)$ is $(1, n)$, where $n+1=h^{(1,1)}(B)$, i.e. has one positive and $n$ negative eigenvalues.

(5) positivity of the Kähler parameters $j_{i}$ and validity of the large volume approximation, i.e. $j_{i} \gg 1$ for all $i$.

We claim that the proof presented below applies to all bases $B$ that obey these conditions.

We note that properties (4) and (5) are automatically satisfied for all the surfaces we consider: the signature of the matrix $C_{i j}$ defined in $(2.8)$ is $(1, n)$, cf. section 2.3 , and $j_{i} \gg 1$ always holds in the Kähler cone basis at large volume for any $B$. The validity of properties (1)-(3) for the considered bases is shown in the appendices A and B. As discussed there, the only subtlety arises for the higher del Pezzos $d P_{n}, n>2$, which have non-simplicial Kähler cones. In this case, the indices $i$ refer to the generators of a suitably chosen simplicial subcone, such that properties (1)-(3) hold. As argued in appendix A there always exists a covering of the Kähler cones of the $d P_{n}$ by simplicial subcones, such that for each subcone in the covering properties (1)-(3) hold.

The following proof is organized as follows. We already introduced the two types of branes, denoted $\beta$ - and $\gamma$-branes, to distinguish between branes with and without fluxes along the fiber $\mathcal{E}$, i.e. $\int_{\mathcal{E}} F^{\beta} \neq 0$ and $\int_{\mathcal{E}} F^{\gamma}=0$, respectively. First we prove in section 3.3 .1 that there is only a finite number of flux configurations on $\beta$-branes. Then in section 3.3.2 we show finiteness of the numbers of D5-branes $n_{I}^{\mathrm{D} 5}$. Finally, we conclude the proof in section 3.3.3 by showing finiteness of the number of flux configurations on $\gamma$-branes.

\subsubsection{Bounds on $\beta$-branes}

Bounds on $m_{0}^{\beta}$. In the following we obtain a bound on the flux component $m_{0}^{\beta}$ for all $\beta$-branes. The result is

$$
\left|m_{0}^{\beta}\right| \leq \max \left(T_{i}\right),
$$

where the maximum is taken over all generators of the specific subcone of the Kähler cone. We note that here and in the rest of the paper, all minima and maxima on $T_{i}$ and $x_{\{i, k\}}$ are taken across generators of the specific subcone we are in. However, except the minimum on $T_{i}$ in theorem 4 , the reader is free to take all other maxima and minima across all generators of the entire Kähler cone, for easy computation purposes. For del Pezzo surfaces this yields $\max \left(T_{i}\right)=36$, for the Hirzebruch surfaces $\mathbb{F}_{k}$ it is $\max \left(T_{i}\right)=24+12 k$ and for the toric surfaces we can read off this bound from table 5 .

We begin by considering inequality (3.7). In fact, since $\mathcal{K}_{000} \geq 0$, (3.7) implies

$$
0>\sum_{i}^{p} t_{i}^{\beta}\left(\frac{b_{i}}{2}+\frac{j_{i}}{j_{0}}\right)
$$

Next we multiply this by $N^{\beta}\left(m_{0}^{\beta}\right)^{2}$ and sum over $\beta$ to obtain, using (3.3),

$$
0>\sum_{\beta} \sum_{i}^{p} N^{\beta} t_{i}^{\beta}\left(m_{0}^{\beta}\right)^{2}\left(\frac{b_{i}}{2}+\frac{j_{i}}{j_{0}}\right)=\sum_{i}^{p}\left(n_{i}^{\mathrm{D} 5}-T_{i}\right)\left(\frac{b_{i}}{2}+\frac{j_{i}}{j_{0}}\right) \geq \sum_{i}^{p}\left(-T_{i}\right)\left(\frac{b_{i}}{2}+\frac{j_{i}}{j_{0}}\right)
$$


where we set the positive $n_{i}^{\mathrm{D} 5}=0$ for all $i$ in the last inequality. This lower bound on the sum over $\beta$ also implies

$$
0>\sum_{i=1}^{p} N_{\beta} t_{i}^{\beta}\left(m_{0}^{\beta}\right)^{2}\left(\frac{b_{i}}{2}+\frac{j_{i}}{j_{0}}\right) \geq \sum_{i=1}^{p}\left(-T_{i}\right)\left(\frac{b_{i}}{2}+\frac{j_{i}}{j_{0}}\right) .
$$

because by (3.20) all summands are negative. This motivates the following definition:

Definition 1. A special brane is a $\beta$-brane with $t_{i}^{\beta}<0$ for all $i$. A mixed brane is a $\beta$-brane which is not a special brane (i.e. there exists an $i$ such that $t_{i}^{\beta} \geq 0$ ).

Remark 1. By (3.20), there does not exist a mixed brane with $t_{i} \geq 0 \forall i$, since $b_{i}, j_{i} \geq 0$. Hence for a mixed brane, we cannot have $t_{i}$ of the same sign $\forall i$, they must be of mixed signs. This motivates its name.

For special branes, we immediately conclude from (3.22) that

$$
\begin{aligned}
\max \left(T_{i}\right) \sum_{i=1}^{p}\left(\frac{b_{i}}{2}+\frac{j_{i}}{j_{0}}\right) & \geq \sum_{i=1}^{p} T_{i}\left(\frac{b_{i}}{2}+\frac{j_{i}}{j_{0}}\right) \geq \sum_{i=1}^{p} N_{\beta}\left|t_{i}^{\beta}\right|\left(m_{0}^{\beta}\right)^{2}\left(\frac{b_{i}}{2}+\frac{j_{i}}{j_{0}}\right) \\
& =\sum_{i=1}^{p} \underbrace{N_{\beta}\left|t_{i}^{\beta} m_{0}^{\beta}\right|}_{\in \mathbb{N}, \geq 1}\left|m_{0}^{\beta}\right|\left(\frac{b_{i}}{2}+\frac{j_{i}}{j_{0}}\right) \geq\left|m_{0}^{\beta}\right| \sum_{i=1}^{p}\left(\frac{b_{i}}{2}+\frac{j_{i}}{j_{0}}\right) .
\end{aligned}
$$

Here we have used (3.22) in the second inequality, and that $t_{i}^{\beta} m_{0}^{\beta}=\sum_{j} C_{i j}\left(b_{j} m_{0}^{\beta}+2 m_{j}^{\beta}\right)$ is a non-zero positive integer, cf. (3.3) in the last inequality: it is an integer because both its first term, $\mathcal{K}_{00 i} m_{0}^{\beta}$, and the second term, the flux $F^{\beta}$ integrated over the integral class $D_{i}$, are integers by (2.12). It is non-zero because $t_{i}^{\beta}$ is non-zero by the definition of special branes, and $m_{0}^{\beta}$ is non-zero by the definition of $\beta$-branes. Thus for special branes, the flux quantum $m_{0}^{\beta}$ is bounded as

$$
\left|m_{0}^{\beta}\right| \leq \max \left(T_{i}\right) .
$$

We will show that mixed branes have a even smaller bound for their $\left|m_{0}^{\beta}\right|$.

Let us first make an observation that will facilitate the identification of special branes.

Lemma 1. A $\beta$-brane which satisfies $0 \leq C\left(\frac{b}{2}+\frac{j}{j_{0}}, b+\frac{m^{\beta}}{m_{0}^{\beta}}\right)$ is a special brane.

Proof. For any $\beta$ brane with $0 \leq C\left(\frac{b}{2}+\frac{j}{j_{0}}, b+\frac{m^{\beta}}{m_{0}^{\beta}}\right)$, consider its SUSY equality (3.1). Then

$$
\text { 1.h.s. of }(3.1) \geq 3 C\left(\frac{j}{j_{0}}, \frac{j}{j_{0}}\right) j_{0}^{2} \text {. }
$$

Suppose it is not a special brane. Then by definition we cannot have $t_{i}^{\beta}<0 \forall i$. Remark 1 also forbids $t_{i}^{\beta} \geq 0 \forall i$. Thus there exists a pair of $i, k$ such that $t_{i}^{\beta}$ and $t_{k}^{\beta}$ are of opposite signs (the following argument still applies if one of them is zero). Writing the r.h.s. of (3.1) in terms of this particular pair of $t_{i}^{\beta}, t_{k}^{\beta}$, we observe that

$$
\text { r.h.s. of }(3.1) \leq \frac{1}{4} \mathcal{K}_{000}\left(m_{0}^{\beta}\right)^{2} \leq \frac{1}{4} \mathcal{K}_{000} \sum_{\beta} N^{\beta}\left(m_{0}^{\beta}\right)^{2}<\frac{3}{2} \sum_{i=1}^{p}\left(T_{i}\right)\left(\frac{b_{i}}{2}+\frac{j_{i}}{j_{0}}\right)
$$


where in the first inequality we dropped all negative terms on the r.h.s. of (3.1) and in the last inequality we employed the lower bound on (3.8). Now (3.25) shows that the l.h.s. of (3.1) is at least quadratic in the $j_{i}$ 's and grows as the Kähler volume of $B$. However, inequality (3.26) implies that the r.h.s. of (3.1) is at most on the order of $j_{i} / j_{0}$. In the limit of all $j_{I}$ large, which in particular implies large volume of $B$, the l.h.s. of (3.1) has to be greater than the r.h.s. of (3.1). Thus, the SUSY equality (3.1) is violated. Our initial assumption that this $\beta$-brane is not a special brane must be wrong; it must be a special brane.

Remark 2. The argument in lemma 1 about the growth of the two sides of the SUSY equality (3.1) can be further substantiated for concrete bases $B$. For all $\mathbb{F}_{k}$, we can check that we have 1.h.s. of $(3.1)>$ r.h.s. of (3.1) when $j_{I} \geq 3 \forall I$. This is clearly the case if the supergravity approximation is supposed to be valid. For $d P_{n}$, the matrix $C(\cdot, \cdot)$ has signature $(1, n)$, i.e. we can have $C(j, j)=0$ for $j \neq 0$ and the above argument might be invalidated. However, we can only have $C(j, j)=0$ if the Kähler form $j_{B}=\sum_{i} j_{i} D_{i}$ on $B$ is on the boundary of the Kähler cone. This means that the Kähler volume of $B$ is zero or cycles in $B$ have shrunk to zero which clearly invalidates the supergravity approximation.

Thus, it remains to bound $m_{0}^{\beta}$ for $\beta$-branes satisfying $0 \geq C\left(\frac{b}{2}+\frac{j}{j_{0}}, b+\frac{m^{\beta}}{m_{0}^{\beta}}\right)$. For such $\beta$-branes, we observe

$$
0 \geq C\left(\frac{b}{2}+\frac{j}{j_{0}}, b+\frac{m^{\beta}}{m_{0}^{\beta}}\right)=\frac{1}{2} \sum_{i=1}^{p} \mathcal{K}_{00 i}\left(\frac{b_{i}}{2}+\frac{j_{i}}{j_{0}}\right)+\underbrace{\frac{1}{2} \underbrace{p}_{i=1} t_{i}^{\beta}\left(\frac{b_{i}}{2}+\frac{j_{i}}{j_{0}}\right)}_{<0 \text { by }(3.20)}
$$

using (2.7) and the definition of $t_{i}^{\beta}(3.3)$. Next, label all $\beta$-branes with $0 \geq C\left(\frac{b}{2}+\frac{j}{j_{0}}, b+\frac{m^{\beta}}{m_{0}^{\beta}}\right)$ by $\beta^{\prime}$, multiply the above inequality by $N^{\beta^{\prime}}\left(m_{0}^{\beta^{\prime}}\right)^{2}$ and sum over $\beta^{\prime}$ :

$$
\begin{aligned}
0 & \geq \frac{1}{2} \sum_{i=1}^{p} \mathcal{K}_{00 i}\left(\frac{b_{i}}{2}+\frac{j_{i}}{j_{0}}\right) \sum_{\beta^{\prime}} N^{\beta^{\prime}}\left(m_{0}^{\beta^{\prime}}\right)^{2}+\frac{1}{2} \sum_{\beta^{\prime}} N^{\beta^{\prime}}\left(m_{0}^{\beta^{\prime}}\right)^{2} \sum_{i=1}^{p} t_{i}^{\beta^{\prime}}\left(\frac{b_{i}}{2}+\frac{j_{i}}{j_{0}}\right) \\
& \geq \frac{1}{2} \sum_{i=1}^{p} \mathcal{K}_{00 i}\left(\frac{b_{i}}{2}+\frac{j_{i}}{j_{0}}\right) \sum_{\beta^{\prime}} N^{\beta^{\prime}}\left(m_{0}^{\beta^{\prime}}\right)^{2}+\frac{1}{2} \sum_{\beta} N^{\beta}\left(m_{0}^{\beta}\right)^{2} \sum_{i=1}^{p} t_{i}^{\beta}\left(\frac{b_{i}}{2}+\frac{j_{i}}{j_{0}}\right) \\
& =\frac{1}{2} \sum_{i=1}^{p} \mathcal{K}_{00 i}\left(\frac{b_{i}}{2}+\frac{j_{i}}{j_{0}}\right) \sum_{\beta^{\prime}} N^{\beta^{\prime}}\left(m_{0}^{\beta^{\prime}}\right)^{2}+\frac{1}{2} \sum_{i=1}^{p}\left(n_{i}^{D 5}-T_{i}\right)\left(\frac{b_{i}}{2}+\frac{j_{i}}{j_{0}}\right) \\
& \geq \frac{1}{2} \sum_{i=1}^{p} \mathcal{K}_{00 i}\left(\frac{b_{i}}{2}+\frac{j_{i}}{j_{0}}\right) \sum_{\beta^{\prime}} N^{\beta^{\prime}}\left(m_{0}^{\beta^{\prime}}\right)^{2}+\frac{1}{2} \sum_{i=1}^{p}\left(-T_{i}\right)\left(\frac{b_{i}}{2}+\frac{j_{i}}{j_{0}}\right) .
\end{aligned}
$$

Here in the second line we extended the sum over $\beta^{\prime}$ to the sum over all $\beta$-branes; by (3.20) each summand is negative, thus, extending the sum only decreases it. In the third line we have used (3.3). With (2.13) and the last line of the above inequality we obtain

$$
12 \sum_{i=1}^{p} \mathcal{K}_{00 i}\left(\frac{b_{i}}{2}+\frac{j_{i}}{j_{0}}\right) \geq \sum_{\beta^{\prime}} N^{\beta^{\prime}}\left(m_{0}^{\beta^{\prime}}\right)^{2} \sum_{i=1}^{p} \mathcal{K}_{00 i}\left(\frac{b_{i}}{2}+\frac{j_{i}}{j_{0}}\right)
$$




\begin{tabular}{|c|c|c|c|c|}
\hline Branes & $\begin{array}{c}\text { Special branes with } \\
0 \leq C\left(\frac{b}{2}+\frac{j}{j_{0}}, b+\frac{m^{\beta}}{m_{0}^{\beta}}\right)\end{array}$ & $\begin{array}{c}\text { Special branes with } \\
0 \geq C\left(\frac{b}{2}+\frac{j}{j_{0}}, b+\frac{m^{\beta}}{m_{0}^{\beta}}\right)\end{array}$ & Mixed branes & $\gamma$-branes \\
\hline$m_{0}^{\alpha}$-bound & $\left|m_{0}^{\beta}\right| \leq \max \left(T_{i}\right)$ & $\left|m_{0}^{\beta}\right| \leq 3$ & $\left|m_{0}^{\beta}\right| \leq 3$ & $m_{0}^{\gamma}=0$ \\
\hline
\end{tabular}

Table 1. Summary of bounds on $m_{0}^{\alpha}$.

Comparing coefficients, we see $\sum_{\beta^{\prime}} N^{\beta^{\prime}}\left(m_{0}^{\beta^{\prime}}\right)^{2} \leq 12$ which implies the bound

$$
\left|m_{0}^{\beta^{\prime}}\right| \leq 3 .
$$

This is an even smaller bound than (3.24) derived previously for special branes satisfying $0 \leq C\left(\frac{b}{2}+\frac{j}{j_{0}}, b+\frac{m^{\beta}}{m_{0}^{\beta}}\right)$ because each $T_{i}=12 \mathcal{K}_{00 i}$ is a integer multiple of 12 . Thus, the overall bound on $m_{0}^{\beta}$ for a $\beta$-brane is still $\left|m_{0}^{\beta}\right| \leq \max \left(T_{i}\right)$.

Recall $\gamma$ branes by definition have $m_{0}^{\gamma}=0$. Thus we are done bounding $m_{0}^{\alpha}$, where (3.19) is the concrete, computable bound. In summary, we have found the precise bounds in table 1 .

Bounds on the number of solutions to the vector $\boldsymbol{m}^{\boldsymbol{\beta}}$. We begin by noting that (3.3) can be viewed as the following matrix multiplication equation

$$
t^{\beta}:=\left(\begin{array}{c}
t_{1}^{\beta} \\
\cdot \\
\cdot \\
\cdot \\
t_{p}^{\beta}
\end{array}\right)=2 C \cdot\left(\frac{b}{2}+\frac{m^{\beta}}{m_{0}^{\beta}}\right) .
$$

The invertible matrix $2 C$ gives a 1-1 correspondence between the vector $m^{\beta}$ and the vector $t^{\beta}$. Thus, in order to show that there are finitely many solutions for the vector $m^{\beta}$, we can equivalently show that there are finitely many solutions for the vector $t^{\beta}$.

We can accomplish this by showing each component $t_{i}^{\beta}$ is bounded. We recall that it suffices to prove each $t_{i}^{\beta}$ is bounded above: since $\left(m_{0}^{\beta}\right)^{2}$ is bounded as we have just shown, an upper bound also implies a lower bound by the second inequality in (3.4). Since the $t_{i}^{\beta}$ of special branes are by definition bounded above by 0 , see definition 1 , we only have to bound the $t_{i}^{\beta}$ of mixed branes.

It is important for finding this upper bound on the $t_{i}^{\beta}$, to first analyze how each type of branes contribute to the sign of a tadpole. We obtain the table 2 , where we have indicated in parenthesis where the corresponding result will be proven in this work.

Next, we proceed with proving the results of this table. We begin with the following

Proposition 1. $\gamma$-branes only contribute negatively to the $0^{\text {th }}$-tadpole (3.2). Furthermore, any $\gamma$-brane contributing zero to the $0^{\text {th }}$-tadpole is the trivial brane, i.e. $m_{I}^{\gamma}=0$ for all $I$. 


\begin{tabular}{|c|c|c|c|c|}
\hline & \multicolumn{2}{|c|}{ Special branes } & Mixed branes & $\gamma$-branes \\
\hline $0^{\text {th }}$-tadpole & $\begin{array}{c}\text { positive } \\
\left(\Rightarrow \forall \tilde{t}_{i}^{\beta}<0 \text { by Cor. 1) }\right.\end{array}$ & negative & $\begin{array}{c}\text { negative } \\
\text { (by Prop. 2) }\end{array}$ & $\begin{array}{c}\text { negative } \\
\text { (by Prop. 1) }\end{array}$ \\
\hline$i^{\text {th }}$-tadpole & $\begin{array}{c}\text { negative } \\
\text { (by (3.3) and Def. 1) }\end{array}$ & 0 \\
& \multicolumn{2}{|c|}{$\left(t_{i}^{\beta}\right)$} & \\
\hline
\end{tabular}

Table 2. Summary of the contributions of the different types of branes to the different tadpoles.

Proof. A $\gamma$-brane's contribution to the $0^{\text {th }}$-tadpole is proportional to $C\left(m^{\gamma}, m^{\gamma}\right)$ by (3.2). In addition, for $\gamma$-branes, the SUSY equality in (2.4) reads

$$
C\left(\left(\frac{b}{2}+\frac{j}{j_{0}}\right), m^{\gamma}\right)=0
$$

as can be seen by setting $m_{0}^{\gamma}=0$ and using the intersection relations $(2.7)$.

We recall that $C$ has Minkowski signature $(1,1)$ for $\mathbb{F}_{k}$ and $(1, n)$ for $d P_{n}$ and the toric surfaces. The vector $\frac{b}{2}+\frac{j}{j_{0}}$ is time-like, since

$$
C\left(\left(\frac{b}{2}+\frac{j}{j_{0}}\right),\left(\frac{b}{2}+\frac{j}{j_{0}}\right)\right)=\frac{1}{4} \mathcal{K}_{000}+\sum_{i=1}^{p} \mathcal{K}_{00 i} \frac{j_{i}}{j_{0}}+C\left(\frac{j}{j_{0}}, \frac{j}{j_{0}}\right)>0 .
$$

Here, the first term on the r.h.s. of (3.33) is positive because $\mathcal{K}_{000}=8$ for $\mathbb{F}_{n}, 9-n$ for $d P_{n}$ and table 5 applies for toric surfaces. The second term is positive because $j_{I}>0$ and for $\mathbb{F}_{k}$, $\mathcal{K}_{001}=2, \mathcal{K}_{002}=2+k ;$ for $d P_{n}, \mathcal{K}_{00 i}=2,3$; for toric surfaces, all relevant entries in table 5 are positive. Finally, the third term is positive because it is proportional to the volume of $B$. By (3.32) the vector $m^{\gamma}$ is orthogonal to a time-like vector, thus, it is space-like, i.e. $0>C\left(m^{\gamma}, m^{\gamma}\right)$, unless it is the zero vector, which trivially has $C\left(m^{\gamma}, m^{\gamma}\right)=0$.

Proposition 2. Only special branes contribute positively to the $0^{\text {th }}$-tadpole. This is equivalent to the fact, that mixed branes only contribute negatively to the $0^{\text {th }}$-tadpole.

Proof. We recall that the $0^{\text {th }}$-tadpole can be written in the form (3.10) for arbitrary choices of $\{i, k\}, i \neq k$. Focusing on its r.h.s., we note that the second term is always negative by the positive semi-definiteness of the matrices $M_{\{i, k\}}$. Furthermore, the third term is always negative by proposition 1 . Thus, the r.h.s. of (3.10) can only be positive, if the first term on the r.h.s. is positive. This implies that all $\tilde{t}_{i}^{\beta}=\mathcal{K}_{00 i}+t_{i}^{\beta}$, cf. (3.11), have to be of the same sign: if not, there exists a pair $\tilde{t}_{i}^{\beta}, \tilde{t}_{k}^{\beta}$ of opposite sign. Writing the r.h.s. of (3.10) in terms of this pair, the first term is negative and the entire r.h.s. of (3.10) would be negative.

If all $\tilde{t}_{i}^{\beta}$ are negative, all $t_{i}^{\beta}$ have to be strictly negative since each $\mathcal{K}_{00 i}$ are strictly positive. By definition 1, a $\beta$-brane with this property is a special brane. If the $\tilde{t}_{i}^{\beta}$ are all positive, then we have $\frac{1}{2} \sum_{i=1}^{p} \tilde{t}_{i}^{\beta}\left(\frac{b_{i}}{2}+\frac{j_{i}}{j_{0}}\right)=C\left(\frac{b}{2}+\frac{j}{j_{0}}, b+\frac{m^{\beta}}{m_{0}^{\beta}}\right) \geq 0$, and by lemma 1 it is also a special brane. 
Corollary 1. A special brane that contributes positively to the $0^{\text {th }}$-tadpole must have $\tilde{t}_{i}^{\beta}<0$ for all $i$.

Proof. Recall from the proof of proposition 1 that a special brane which contributes positively to the $0^{\text {th }}$-tadpole must have all $\tilde{t}_{i}^{\beta}$ of the same sign. If they are all negative, we are done. Thus, assume all $\tilde{t}_{i}^{\beta} \geq 0$. We prove this is not possible using a similar argument as in the proof of lemma 1.

Since $\mathcal{K}_{00 i}>0 \forall i$ and we are considering a special brane, i.e. all $t_{i}^{\beta}<0$, having $\tilde{t}_{i}^{\beta}=\left(\mathcal{K}_{00 i}+t_{i}^{\beta}\right) \geq 0 \forall i$ means $\left|t_{i}^{\beta}\right| \leq \mathcal{K}_{00 i} \forall i$. Now consider the SUSY equality (3.1). Since the $M$-matrix is positive semi-definite, the r.h.s. of (3.1) is at most $\left[\frac{1}{4} \mathcal{K}_{000}+\frac{3}{2} x^{\{i, k\}} \mathcal{K}_{00 i} \mathcal{K}_{00 k}\right]\left(m_{0}^{\beta}\right)^{2}$. Also, by the last inequality in (3.8), we have

$$
6 \sum_{i=1}^{p} T_{i}\left(\frac{b_{i}}{2}+\frac{j_{i}}{j_{0}}\right)>\mathcal{K}_{000} \sum_{\beta} N_{\beta}\left(m_{0}^{\beta}\right)^{2},
$$

i.e. $\left(m_{0}^{\beta}\right)^{2}$ is smaller than a linear combination of $j_{i} / j_{0}$, so is $\left[\frac{1}{4} \mathcal{K}_{000}+\frac{3}{2} x^{\{i, k\}} \mathcal{K}_{00 i} \mathcal{K}_{00 k}\right]\left(m_{0}^{\beta}\right)^{2}$, since the prefactor $\left[\frac{1}{4} \mathcal{K}_{000}+\frac{3}{2} x^{\{i, k\}} \mathcal{K}_{00 i} \mathcal{K}_{00 k}\right] \sim \mathcal{K}_{000}$. However, $\tilde{t}_{i}^{\beta}>0$ for all $i$ means $\frac{1}{2} \sum_{i=1}^{p} \tilde{t}_{i}^{\beta}\left(\frac{b_{i}}{2}+\frac{j_{i}}{j_{0}}\right)=C\left(\frac{b}{2}+\frac{j}{j_{0}}, b+\frac{m^{\beta}}{m_{0}^{\beta}}\right) \geq 0$, which implies that the l.h.s. of $(3.1)$ is at least $3 C(j, j)$ which is quadratic in the $j_{i}$.

Thus, in the limit that all $j_{I}$ are large, the l.h.s. of (3.1) will always be greater than its r.h.s., thus violating the SUSY equality. ${ }^{13}$

This concludes the proof of the results in table 2 . We prove three more important lemmas before we finally derive the bounds on $t_{i}^{\beta}$.

For the rest of the proof, we will label special branes that contribute positively to the $0^{\text {th }}$-tadpole by $\beta_{s}$, and mixed branes by $\beta_{m}$. We also use the simplified notation

$$
\sum_{\beta_{m},+} \equiv \sum_{\beta_{m}, \tilde{t}_{i}^{\beta_{m}} \geq 0}
$$

The index $i$ is omitted in this simplified notation when it is clear from the context to which $i$ we are referring.

Lemma 2. For any index $i$, we have the following inequality:

$$
\sum_{\beta_{s}} N^{\beta_{s}}\left|\tilde{t}_{i}^{\beta_{s}}\right|\left(m_{0}^{\beta_{s}}\right)^{2}<\sum_{\beta_{m},+} N^{\beta_{m}} \tilde{t}_{i}^{\beta_{m}}\left(m_{0}^{\beta_{m}}\right)^{2}+T_{i}
$$

Proof. By (3.4), we have a lower bound for the $i^{\text {th }}$-tadpole. Thus, we have the following inequality for the $i^{\text {th }}$-tadpole:

$$
T_{i} \geq \sum_{\beta, t_{i}^{\beta}<0} N^{\beta}\left|t_{i}^{\beta}\right|\left(m_{0}^{\beta}\right)^{2}-\sum_{\beta_{m}, t_{i}^{\beta_{m}} \geq 0} N^{\beta_{m}} t_{i}^{\beta_{m}}\left(m_{0}^{\beta_{m}}\right)^{2} \geq \sum_{\beta_{s}} N^{\beta_{s}}\left|t_{i}^{\beta_{s}}\right|\left(m_{0}^{\beta_{s}}\right)^{2}-\sum_{\beta_{m}, t_{i}^{\beta_{m}} \geq 0} N^{\beta_{m}} t_{i}^{\beta_{m}}\left(m_{0}^{\beta_{m}}\right)^{2}
$$

\footnotetext{
${ }^{13}$ The precise value of the $j_{I}$ at which the SUSY equality is violated can be computed as mentioned in remark 2. For example, for $\mathbb{F}_{k}$, we find that the SUSY equality is violated for $j_{I} \geq 10 \forall I$.
} 


$$
\begin{aligned}
> & \sum_{\beta_{s}} N^{\beta_{s}}\left|t_{i}^{\beta_{s}}\right|\left(m_{0}^{\beta_{s}}\right)^{2}-\sum_{\beta_{m}, t_{i}^{\beta_{m}} \geq 0} N^{\beta_{m}} t_{i}^{\beta_{m}}\left(m_{0}^{\beta_{m}}\right)^{2}-\sum_{\beta_{s}} N^{\beta_{s}} \mathcal{K}_{00 i}\left(m_{0}^{\beta_{s}}\right)^{2}-\sum_{\beta_{m}, t_{i}^{\beta_{m}} \geq 0} N^{\beta_{m}} \mathcal{K}_{00 i}\left(m_{0}^{\beta_{m}}\right)^{2} \\
& -\sum_{\beta_{m}, t_{i}^{\beta_{m}}<0, \tilde{t}_{i}^{\beta_{m}} \geq 0} N^{\beta_{m}} \tilde{t}_{i}^{\beta_{m}}\left(m_{0}^{\beta_{m}}\right)^{2} \\
= & \sum_{\beta_{s}} N^{\beta_{s}}\left|\tilde{t}_{i}^{\beta_{s}}\right|\left(m_{0}^{\beta_{s}}\right)^{2}-\sum_{\beta_{m}, t_{i}^{\beta_{m}} \geq 0} N^{\beta_{m}} \tilde{t}_{i}^{\beta_{m}}\left(m_{0}^{\beta_{m}}\right)^{2}-\sum_{\beta_{m}, t_{i}^{\beta_{m}}<0, \tilde{t}_{i}^{\beta_{m}} \geq 0} N^{\beta_{m}} \tilde{t}_{i}^{\beta_{m}}\left(m_{0}^{\beta_{m}}\right)^{2} \\
= & \sum_{\beta_{s}} N^{\beta_{s}}\left|\tilde{t}_{i}^{\beta_{s}}\right|\left(m_{0}^{\beta_{s}}\right)^{2}-\sum_{\beta_{m},+} N^{\beta_{m}} \tilde{t}_{i}^{\beta_{m}}\left(m_{0}^{\beta_{m}}\right)^{2},
\end{aligned}
$$

where in the first inequality, we split terms in the sum of (3.4) into positive and negative contributions, as indicated in the summation by $t_{i}^{\beta_{m}} \geq 0$ and $t_{i}^{\beta_{m}}<0$. In the second inequality, in the first term, we only kept those special branes in the sum that contribute positively to the $0^{\text {th }}$-tadpole, which are labelled by $\beta_{s}$. In the second line, we added three more negative terms and in the next equality, we combined them into three sums using (3.11), that yield the two sums in the last line.

Lemma 3. For any pair of a special brane that contributes positively to the $0^{\text {th }}$-tadpole and a mixed brane, there exists an index $k$ such that $\tilde{t}_{k}^{\beta_{m}}$ is strictly negative and $\left|\tilde{t}_{k}^{\beta_{m}}\right|>\left|\tilde{t}_{k}^{\beta_{s}}\right|$. In particular

$$
\left|\tilde{t}_{k}^{\beta_{m}}\right|-\left|\tilde{t}_{k}^{\beta_{s}}\right| \geq \frac{1}{3}
$$

Proof. Suppose the converse is true, i.e. for some pair of a special brane that contributes positively to the $0^{\text {th }}$-tadpole and a mixed brane, there does not exist an index $k$ such that $\tilde{t}_{k}^{\beta_{m}}$ is strictly negative and $\left|\tilde{t}_{k}^{\beta_{m}}\right|>\left|\tilde{t}_{k}^{\beta_{s}}\right|$. Then, consider the difference of the SUSY equality (3.1) for the mixed brane and for the special brane:

$$
\begin{aligned}
& \text { l.h.s. of (3.1) for the mixed brane - l.h.s. of (3.1) for the special brane } \\
= & \text { r.h.s. of (3.1) for the mixed brane - r.h.s. of (3.1) for the special brane }
\end{aligned}
$$

We will show that (3.39) will be violated. To simplify our notation, we will in the following denote the difference of the l.h.s. and r.h.s. in (3.39) by $\Delta_{\text {l.h.s. }}$ and $\Delta_{\text {r.h.s. }}$, respectively. First consider the difference $\Delta_{\text {l.h.s. }}$. The first term, $3 C\left(\frac{j}{j_{0}}, \frac{j}{j_{0}}\right) j_{0}^{2}$, is the same for both branes. Thus, by expanding everything out and using (3.3) and (3.11), we obtain

$$
\begin{aligned}
\Delta_{\text {l.h.s. }} & =6 C\left(\frac{b}{2}+\frac{j}{j_{0}}, b+\frac{m^{\beta_{m}}}{m_{0}^{\beta_{m}}}\right) j_{0}^{2}-6 C\left(\frac{b}{2}+\frac{j}{j_{0}}, b+\frac{m^{\beta_{s}}}{m_{0}^{\beta_{s}}}\right) j_{0}^{2} \\
& =3 \sum_{i=1}^{p} \tilde{t}_{i}^{\beta_{m}}\left(\frac{b_{i}}{2}+\frac{j_{i}}{j_{0}}\right) j_{0}^{2}-3 \sum_{i=1}^{p} \tilde{t}_{i}^{\beta_{s}}\left(\frac{b_{i}}{2}+\frac{j_{i}}{j_{0}}\right) j_{0}^{2} .
\end{aligned}
$$

By corollary 1, since the special brane contributes positively to the $0^{\text {th }}$-tadpole, $\tilde{t}_{i}^{\beta_{s}}<0$ for all $i$. Also notice that the mixed brane must have at least one $i$ for which $\tilde{t}_{i}^{\beta_{m}}>0$, because by definition, a mixed brane must have at least one $i$ for which $t_{i}^{\beta_{m}} \geq 0$, and for 
this $i$, by (3.11) and the positivity of $\mathcal{K}_{00 i}, \tilde{t}_{i}^{\beta_{m}}>0$. Labelling those $i$ for which $\tilde{t}_{i}^{\beta_{m}}>0$ as $i+$, and those $i$ for which $\tilde{t}_{i}^{\beta_{m}} \leq 0$ as $i-,(3.40)$ becomes

$$
\begin{aligned}
\Delta_{\text {l.h.s. }} & =3 j_{0}^{2}\left[\sum_{i+} \tilde{t}_{i+}^{\beta_{m}}\left(\frac{b_{i+}}{2}+\frac{j_{i+}}{j_{0}}\right)+\sum_{i-}\left(\left|\tilde{t}_{i-}^{\beta_{s}}\right|-\left|\tilde{t}_{i-}^{\beta_{m}}\right|\right)\left(\frac{b_{i-}}{2}+\frac{j_{i-}}{j_{0}}\right)+\sum_{i+}\left|\tilde{t}_{i+}^{\beta_{s}}\right|\left(\frac{b_{i+}}{2}+\frac{j_{i+}}{j_{0}}\right)\right] \\
& \geq 3 j_{0}^{2}\left[\sum_{i+} \tilde{t}_{i+}^{\beta_{m}}\left(\frac{b_{i+}}{2}+\frac{j_{i+}}{j_{0}}\right)+\sum_{i+}\left|\tilde{t}_{i+}^{\beta_{s}}\right|\left(\frac{b_{i+}}{2}+\frac{j_{i+}}{j_{0}}\right)\right]
\end{aligned}
$$

Here in the last step we dropped the second sum, which is positive, because by assumption there does not exist an index $k$ such that $\tilde{t}_{k}^{\beta_{m}}$ is strictly negative and $\left|\tilde{t}_{k}^{\beta_{m}}\right|>\left|\tilde{t}_{k}^{\beta_{s}}\right|$.

Notice, by (3.3), $\tilde{t}_{i}^{\beta_{s}}, \tilde{t}_{i}^{\beta_{m}}$ are rational numbers $\frac{a}{m_{0}^{\beta_{s}}}, \frac{b}{m_{0}^{\beta_{m}}}$ with $a, b \in 2 \mathbb{Z}^{14}$ By table 1 , we have $\left|m_{0}^{\beta_{m}}\right| \leq 3$. For the special brane, since $\tilde{t}_{i}^{\beta_{s}}<0$ for all $i$, we have

$$
0>\frac{1}{2} \sum_{i=1}^{p} \tilde{t}_{i}^{\beta_{s}}\left(\frac{b_{i}}{2}+\frac{j_{i}}{j_{0}}\right)=C\left(\frac{b}{2}+\frac{j}{j_{0}}, b+\frac{m^{\beta_{s}}}{m_{0}^{\beta_{s}}}\right) .
$$

Thus, the bound $\left|m_{0}^{\beta_{s}}\right| \leq 3$ in the third column of table 1 applies. This implies both $\left|\tilde{t}_{i}^{\beta_{s}}\right|>0, \tilde{t}_{i+}^{\beta_{m}}>0$ are either integers or a third of integers:

$$
3 \tilde{t}_{i+}^{\beta_{m}}>1, \quad 3\left|\tilde{t}_{i}^{\beta_{s}}\right|>1 .
$$

Hence, (3.41) becomes

$$
\begin{aligned}
\Delta_{\text {l.h.s. }} & \geq \sum_{i+} 3 \tilde{t}_{i+}^{\beta_{m}}\left(\frac{b_{i+}}{2}+\frac{j_{i+}}{j_{0}}\right) j_{0}^{2}+\sum_{i+} 3\left|\tilde{t}_{i+}^{\beta_{s}}\right|\left(\frac{b_{i+}}{2}+\frac{j_{i+}}{j_{0}}\right) j_{0}^{2} \geq 2 \sum_{i+}\left(\frac{b_{i+}}{2}+\frac{j_{i+}}{j_{0}}\right) j_{0}^{2} \\
& \geq 2 \sum_{i+} j_{i+} j_{0}
\end{aligned}
$$

where in the last step we dropped the term containing the positive $b_{i}$. We have discussed that at least one index $i+$ exists. With $j_{I} \gg 1$ for all $I$, (3.44) shows that the difference between the l.h.s. of (3.1) for the two branes is large.

Next, we show that the difference $\Delta_{\text {r.h.s. }}$ between the r.h.s. of (3.1) for the two branes is much smaller. Starting from the r.h.s. of (3.10) for the special brane we note the identity

$$
\begin{aligned}
& 3\left[\frac{1}{2} x_{\{i, k\}} \tilde{t}_{i}^{\beta} \tilde{t}_{k}^{\beta}-M_{\{i, k\}}\left(b+\frac{m^{\beta}}{m_{0}^{\beta}}, b+\frac{m^{\beta}}{m_{0}^{\beta}}\right)\right]\left(m_{0}^{\beta}\right)^{2} \\
& =\left[\frac{1}{4} \mathcal{K}_{000}+\frac{3}{2} x_{\{i, k\}} t_{i}^{\beta} t_{k}^{\beta}-3 M_{\{i, k\}}\left(\frac{b}{2}+\frac{m^{\beta}}{m_{0}^{\beta}}, \frac{b}{2}+\frac{m^{\beta}}{m_{0}^{\beta}}\right)\right]\left(m_{0}^{\beta}\right)^{2}+\frac{3}{2} \sum_{i=1}^{p} b_{i} \tilde{t}_{i}^{\beta}\left(m_{0}^{\beta}\right)^{2}-\mathcal{K}_{000}\left(m_{0}^{\beta}\right)^{2} .
\end{aligned}
$$

Since the special brane contributes positively to the $0^{\text {th }}$-tadpole, the l.h.s. of $(3.45)$ is positive. We also recall that $\tilde{t}_{i}^{\beta}<0$ for all $i$ by corollary 1 , which implies that the second

\footnotetext{
${ }^{14} \mathrm{By}(3.3)$, we have $\tilde{t}_{k}^{\beta_{s}}=2 \mathcal{K}_{00 k}+2 \sum_{j} C_{k j} \frac{m_{j}^{\beta_{s}}}{m_{0}^{\beta_{s}}}=\frac{a}{m_{0}^{\beta_{s}}}, \tilde{t}_{k}^{\beta_{m}}=2 \mathcal{K}_{00 k}+2 \sum_{j} C_{k j} \frac{m_{j}^{\beta_{m}}}{m_{0}^{\beta_{m}}}=\frac{b}{m_{0}^{\beta_{m}}}$ for $a, b \in 2 \mathbb{Z}$.
} 
last term on the r.h.s. of (3.45) is strictly negative, as $b_{i} \geq 0$. In addition, the last term on the r.h.s. is always negative for the bases $B$ we consider. Thus, the term in square brackets on the r.h.s. of (3.45), which is the r.h.s. of (3.1), must be strictly positive. In particular, it must have a bigger magnitude than that of (the next to last term and) the last term:

$$
\text { r.h.s. of (3.1) for the special brane }>\mathcal{K}_{000}\left(m_{0}^{\beta_{s}}\right)^{2} \text {. }
$$

Next, consider the r.h.s. of (3.1) for the mixed brane. Since it is a mixed brane, we can pick a pair of $t_{i}^{\beta_{m}}, t_{k}^{\beta_{m}}$ of opposite signs to make the second term of the r.h.s. of (3.1) negative. By the positive semi-definiteness of the $M$-matrix, the third term of the r.h.s. of (3.1) is always negative. Thus

$$
\text { r.h.s. of (3.1) for the mixed brane } \leq \frac{1}{4} \mathcal{K}_{000}\left(m_{0}^{\beta_{m}}\right)^{2} .
$$

Hence, we obtain, using again the bounds on $m_{0}^{\beta}$ from table 1 ,

$$
\Delta_{\text {r.h.s. }}<\frac{1}{4} \mathcal{K}_{000}\left(m_{0}^{\beta_{m}}\right)^{2}-\mathcal{K}_{000}\left(m_{0}^{\beta_{s}}\right)^{2} \leq \frac{1}{4} \mathcal{K}_{000}(3)^{2}-\mathcal{K}_{000}(1)^{2}=\frac{5}{4} \mathcal{K}_{000}
$$

By comparison of (3.44) and (3.46), using the property $j_{I} \gg 1$ for all $I$, we see that we will always have

$$
\Delta_{\text {l.h.s. }}>\Delta_{\text {r.h.s. }},
$$

which clearly violates (3.39).

Finally we prove (3.38). Recall both $\tilde{t}_{k}^{\beta_{s}}, \tilde{t}_{k}^{\beta_{m}}$ are either integers or a third of an integer. Since $\left|\tilde{t}_{k}^{\beta_{m}}\right|>\left|\tilde{t}_{k}^{\beta_{s}}\right|$, their difference is at least a non-zero integer divided by their common denominator, which is 3, i.e. (3.38) applies.

We make two useful definitions for the next lemma before stating it. Recall that the contribution of a mixed brane to the $0^{\text {th }}$-tadpole is negative, cf. table 2 , and is given by the first term in (3.10):

$$
0 \geq R^{\beta_{m}} \equiv N^{\beta_{m}}\left[\frac{1}{2} x_{\{i, k\}} \tilde{t}_{i}^{\beta_{m}} \tilde{t}_{k}^{\beta_{m}}-M_{\{i, k\}}\left(b+\frac{m^{\beta_{m}}}{m_{0}^{\beta_{m}}}, b+\frac{m^{\beta_{m}}}{m_{0}^{\beta_{m}}}\right)\right]\left(m_{0}^{\beta_{m}}\right)^{2} .
$$

Similarly, for a special brane that contributes positively to the $0^{\text {th }}$-tadpole, its contribution is also given by the first term in (3.10):

$$
0 \leq S^{\beta_{s}} \equiv N^{\beta_{s}}\left[\frac{1}{2} x_{\{i, k\}} \tilde{t}_{i}^{\beta_{s}} \tilde{t}_{k}^{\beta_{s}}-M_{\{i, k\}}\left(b+\frac{m^{\beta_{s}}}{m_{0}^{\beta_{s}}}, b+\frac{m^{\beta_{s}}}{m_{0}^{\beta_{s}}}\right)\right]\left(m_{0}^{\beta_{s}}\right)^{2} .
$$

Thus, the total positive contribution to the $0^{\text {th }}$-tadpole, and part of the total negative contributions to the $0^{\text {th }}$ - tadpole from mixed branes with $\tilde{t}_{i}^{\beta_{m}} \geq 0$ are

$$
\sum_{\beta_{s}} S^{\beta_{s}} \geq 0, \quad \sum_{\beta_{m},+} R^{\beta_{m}} \leq 0 .
$$


Lemma 4. Given $h_{1}, h_{2} \in \mathbb{Q}^{+}, 0<h_{1}, h_{2} \leq 1$, so that for some index $i$

$$
h_{1} \sum_{\beta_{m},+} N^{\beta_{m}} \tilde{t}_{i}^{\beta_{m}}\left(m_{0}^{\beta_{m}}\right)^{2}=h_{2} \sum_{\beta_{s}} N^{\beta_{s}}\left|\tilde{t}_{i}^{\beta_{s}}\right|\left(m_{0}^{\beta_{s}}\right)^{2}
$$

holds, then $h_{1} \sum_{\beta_{m},+}\left|R^{\beta_{m}}\right|>h_{2} \sum_{\beta_{s}} S^{\beta_{s}}$. In particular,

$$
h_{1} \sum_{\beta_{m},+}\left|R^{\beta_{m}}\right|-h_{2} \sum_{\beta_{s}} S^{\beta_{s}} \geq \frac{1}{6} \min \left(x_{\{i, k\}}\right) h_{1} \sum_{\beta_{m},+} N^{\beta_{m}} \tilde{t}_{i}^{\beta_{m}}\left(m_{0}^{\beta_{m}}\right)^{2},
$$

where the minimum and maximum is taken over all pairs $\{i, k\}$ of indices of Kähler cone generators in the subcone, but can also be taken across the entire Kähler cone.

Proof. We introduce a partition of unity $\left\{f^{\beta_{s}}\right\}_{\beta_{s}}$, and, for every index $\beta_{s}$, a partition of unity $\left\{g^{\beta_{s}, \beta_{m}}\right\}_{\beta_{m},+},{ }^{15}$ i.e.

$$
\sum_{\beta_{s}} f^{\beta_{s}}=1, \quad \sum_{\beta_{m},+} g^{\beta_{s}, \beta_{m}}=1, \quad f^{\beta_{s}}, g^{\beta_{s}, \beta_{m}} \in \mathbb{Q}^{+}, \quad 0<f^{\beta_{s}}, g^{\beta_{s}, \beta_{m}} \leq 1,
$$

defined by the property

$$
f^{\beta_{s}} h_{1} N^{\beta_{m}} \tilde{t}_{i}^{\beta_{m}}\left(m_{0}^{\beta_{m}}\right)^{2}=g^{\beta_{s}, \beta_{m}} h_{2} N^{\beta_{s}}\left|\tilde{t}_{i}^{\beta_{s}}\right|\left(m_{0}^{\beta_{s}}\right)^{2} .
$$

Inserting unity as $1=\sum_{\beta_{s}} f^{\beta_{s}}=\sum_{\beta_{m},+}, g^{\beta_{s}, \beta_{m}}$, we obtain the obvious identity

$$
\begin{aligned}
& h_{1} \sum_{\beta_{m},+}\left|R^{\beta_{m}}\right|-h_{2} \sum_{\beta_{s}} S^{\beta_{s}}=\left(\sum_{\beta_{s}} f^{\beta_{s}}\right) h_{1} \sum_{\beta_{m},+}\left|R^{\beta_{m}}\right|-h_{2} \sum_{\beta_{s}}\left(\sum_{\beta_{m},+} g^{\beta_{s}, \beta_{m}}\right) S^{\beta_{s}} \\
& =\sum_{\beta_{s}} \sum_{\beta_{m},+}\left(f^{\beta_{s}} h_{1}\left|R^{\beta_{m}}\right|-g^{\beta_{s}, \beta_{m}} h_{2} S^{\beta_{s}}\right) .
\end{aligned}
$$

For each summand in the sum of the last line of (3.55), we have

$$
\begin{gathered}
f^{\beta_{s}} h_{1}\left|R^{\beta_{m}}\right|-g^{\beta_{s}, \beta_{m}} h_{2} S^{\beta_{s}}=f^{\beta_{s}} h_{1} N^{\beta_{m}}|\frac{1}{2} x_{\{i, k\}} \underbrace{\tilde{t}_{i}^{\beta_{m}}}_{\geq 0} \underbrace{\tilde{t}_{k}^{\beta_{m}}}_{<0}-M_{\{i, k\}}\left(b+\frac{m^{\beta_{m}}}{m_{0}^{\beta_{m}}}, b+\frac{m^{\beta_{m}}}{m_{0}^{\beta_{m}}}\right)|\left(m_{0}^{\beta_{m}}\right)^{2} \\
-g^{\beta_{s}, \beta_{m}} h_{2} N^{\beta_{s}}[\frac{1}{2} x_{\{i, k\}} \underbrace{\tilde{t}_{i}^{\beta_{s}}}_{<0} \underbrace{\tilde{t}_{k}^{\beta_{s}}}_{<0}-M_{\{i, k\}}\left(b+\frac{m^{\beta_{s}}}{m_{0}^{\beta_{s}}}, b+\frac{m^{\beta_{s}}}{m_{0}^{\beta_{s}}}\right)]\left(m_{0}^{\beta_{s}}\right)^{2} .
\end{gathered}
$$

Here, the pair $\{i, k\}$ is chosen so that the index $i$ is the one for which (3.51) holds, and the index $k$ is chosen such that the inequality $\left|\tilde{t}_{k}^{\beta_{m}}\right|-\left|\tilde{t}_{k}^{\beta_{s}}\right| \geq \frac{1}{3}$ of lemma 3 holds for the pair $\left(\beta_{s}, \beta_{m}\right)$ of special and mixed brane in (3.56). We emphasize that the choice of this index $k$ depends on the brane pair $\left(\beta_{s}, \beta_{m}\right)$ and thus might be different for each summand in (3.55). Next, we drop the positive semi-definite $M$-matrix terms in (3.56) to get

$$
f^{\beta_{s}} h_{1}\left|R^{\beta_{m}}\right|-g^{\beta_{s}, \beta_{m}} h_{2} S^{\beta_{s}} \geq \frac{1}{2} x_{\{i, k\}}\left[f^{\beta_{s}} h_{1} N^{\beta_{m}} \tilde{t}_{i}^{\beta_{m}}\left(m_{0}^{\beta_{m}}\right)^{2}\left|\tilde{t}_{k}^{\beta_{m}}\right|-g^{\beta_{s}, \beta_{m}} h_{2} N^{\beta_{s}}\left|\tilde{t}_{i}^{\beta_{s}}\right|\left(m_{0}^{\beta_{s}}\right)^{2}\left|\tilde{t}_{k}^{\beta_{s}}\right|\right]
$$

\footnotetext{
${ }^{15}$ We emphasize that the index $\beta_{m}$ on $g^{\beta_{s}, \beta_{m}}$ is only limited to mixed branes with $\tilde{t}_{i}^{\beta_{m}} \geq 0$.
} 


$$
\begin{aligned}
& =\frac{1}{2} x_{\{i, k\}}\left(f^{\beta_{s}} h_{1} N^{\beta_{m}} \tilde{t}_{i}^{\beta_{m}}\left(m_{0}^{\beta_{m}}\right)^{2}\right) \underbrace{\left(\left|\tilde{t}_{k}^{\beta_{m}}\right|-\left|\tilde{t}_{k}^{\beta_{s}}\right|\right)}_{\geq 1 / 3} \\
& \geq \frac{1}{6} \min \left(x_{\{i, k\}}\right) f^{\beta_{s}} h_{1} N^{\beta_{m}} \tilde{t}_{i}^{\beta_{m}}\left(m_{0}^{\beta_{m}}\right)^{2},
\end{aligned}
$$

where we have used that the coefficients of $\left|\tilde{t}_{k}^{\beta_{m}}\right|,\left|\tilde{t}_{k}^{\beta_{s}}\right|$ in the first line are equal by (3.54). In addition, we have removed the aforementioned implicit dependence of the index $k$ on $\left(\beta_{s}, \beta_{m}\right)$ by taking the minimum over all $\{i, k\}$.

Thus, plugging (3.57) into (3.55) we obtain

$$
h_{1} \sum_{\beta_{m},+}\left|R^{\beta_{m}}\right|-h_{2} \sum_{\beta_{s}} S^{\beta_{s}} \geq \frac{1}{6} \min \left(x_{\{i, k\}}\right) h_{1} \sum_{\beta_{m},+} N^{\beta_{m}} \tilde{t}_{i}^{\beta_{m}}\left(m_{0}^{\beta_{m}}\right)^{2},
$$

where we performed the sum over $\beta_{s}$ and used $\sum_{\beta_{s}} f^{\beta_{s}}=1$, cf. (3.53).

Now we are finally ready to show that every $t_{i}^{\beta}$ has an upper bound.

Theorem 2. For all $i$ and $\beta, t_{i}^{\beta}$ are bounded from above as

$$
t_{i}^{\beta} \leq \frac{6 T_{0}+3 T_{i} \cdot \max \left(x_{\{i, k\}}\right) \cdot \max \left(T_{l}\right)}{\min \left(x_{\{i, k\}}\right)},
$$

where the minimum and maximum is taken over all pairs $\{i, k\}$ of indices of Kähler cone generators in the subcone, but can also be taken across the entire Kähler cone.

Proof. We derive the above bound for $t_{i}^{\beta}$ for an arbitrary index $i$. By lemma 2, we either have $\sum_{\beta_{s}} N^{\beta_{s}}\left|\tilde{t}_{i}^{\beta_{s}}\right|\left(m_{0}^{\beta_{s}}\right)^{2} \leq \sum_{\beta_{m},+} N^{\beta_{m}} \tilde{t}_{i}^{\beta_{m}}\left(m_{0}^{\beta_{m}}\right)^{2}$, or $\sum_{\beta_{m},+} N^{\beta_{m}} \tilde{t}_{i}^{\beta_{m}}\left(m_{0}^{\beta_{m}}\right)^{2}<$ $\sum_{\beta_{s}} N^{\beta_{s}}\left|\tilde{t}_{i}^{\beta_{s}}\right|\left(m_{0}^{\beta_{s}}\right)^{2}<\sum_{\beta_{m},+} N^{\beta_{m}} \tilde{t}_{i}^{\beta_{m}}\left(m_{0}^{\beta_{m}}\right)^{2}+T_{i}$. We consider each case separately:

Case 1: $\quad \sum_{\beta_{s}} N^{\beta_{s}}\left|\tilde{t}_{i}^{\beta_{s}}\right|\left(m_{0}^{\beta_{s}}\right)^{2} \leq \sum_{\beta_{m},+} N^{\beta_{m}} \tilde{t}_{i}^{\beta_{m}}\left(m_{0}^{\beta_{m}}\right)^{2}$.

In other words, we have a relation as in (3.51) with $h_{1} \leq 1, h_{2}=1$,

$$
h_{1} \sum_{\beta_{m},+} N^{\beta_{m}} \tilde{t}_{i}^{\beta_{m}}\left(m_{0}^{\beta_{m}}\right)^{2}=\sum_{\beta_{s}} N^{\beta_{s}}\left|\tilde{t}_{i}^{\beta_{s}}\right|\left(m_{0}^{\beta_{s}}\right)^{2} .
$$

Starting with the first inequality in (3.4) and employing (3.48), (3.49), we obtain

$$
\begin{aligned}
T_{0} & \geq \sum_{\beta_{m},+}\left|R^{\beta_{m}}\right|-\sum_{\beta_{s}} S^{\beta_{s}}=\left(1-h_{1}\right) \sum_{\beta_{m},+}\left|R^{\beta_{m}}\right|+h_{1} \sum_{\beta_{m},+}\left|R^{\beta_{m}}\right|-\sum_{\beta_{s}} S^{\beta_{s}} \\
& \geq\left(1-h_{1}\right) \sum_{\beta_{m},+}\left|R^{\beta_{m}}\right|+\frac{1}{6} \min \left(x_{\{i, k\}}\right) h_{1} \sum_{\beta_{m},+} N^{\beta_{m}} \tilde{t}_{i}^{\beta_{m}}\left(m_{0}^{\beta_{m}}\right)^{2} \\
& \geq\left(1-h_{1}\right) \sum_{\beta_{m},+} N^{\beta_{m}} \frac{1}{2} x_{\{i, k\}} \tilde{t}_{i}^{\beta_{m}} \underbrace{\left|\tilde{t}_{k}^{\beta_{m}}\right|}_{\geq 1 / 3}\left(m_{0}^{\beta_{m}}\right)^{2}+\frac{1}{6} \min \left(x_{\{i, k\}}\right) h_{1} \sum_{\beta_{m},+} N^{\beta_{m}} \tilde{t}_{i}^{\beta_{m}}\left(m_{0}^{\beta_{m}}\right)^{2} \\
& \geq\left(1-h_{1}\right) \frac{1}{6} \min \left(x_{\{i, k\}}\right) \sum_{\beta_{m},+} N^{\beta_{m}} \tilde{t}_{i}^{\beta_{m}}\left(m_{0}^{\beta_{m}}\right)^{2}+h_{1} \frac{1}{6} \min \left(x_{\{i, k\}}\right) \sum_{\beta_{m},+} N^{\beta_{m}} \tilde{t}_{i}^{\beta_{m}}\left(m_{0}^{\beta_{m}}\right)^{2}
\end{aligned}
$$




$$
=\frac{1}{6} \min \left(x_{\{i, k\}}\right) \sum_{\beta_{m},+} N^{\beta_{m}} \tilde{t}_{i}^{\beta_{m}}\left(m_{0}^{\beta_{m}}\right)^{2},
$$

where in the first inequality we only kept negative contributions to the $0^{\text {th }}$-tadpole from mixed branes with $\tilde{t}_{i}^{\beta_{m}} \geq 0$ (see table 2). In the second line we used lemma 4 . In the third line we plugged in the definition (3.48) of $R^{\beta_{m}}$, where we picked our choice of the pair $\{i, k\}$ so that $i$ is the same index $i$ that we want to derive a bound for $t_{i}^{\beta}$, and $k$ such that $\left|\tilde{t}_{k}^{\beta_{m}}\right| \geq \frac{1}{3},{ }^{16}$ and dropped the M-matrix term. The remaining two lines of (3.61) are just algebra. Thus, we have the following bound on $t_{i}$ :

$$
T_{0} \geq \frac{1}{6} \min \left(x_{\{i, k\}}\right) \sum_{\beta_{m},+} N^{\beta_{m}} \tilde{t}_{i}^{\beta_{m}}\left(m_{0}^{\beta_{m}}\right)^{2}, \Longrightarrow t_{i}^{\beta}<\sum_{\beta_{m},+} N^{\beta_{m}} \tilde{t}_{i}^{\beta_{m}}\left(m_{0}^{\beta_{m}}\right)^{2} \leq \frac{6 T_{0}}{\min \left(x_{\{i, k\}}\right)}
$$

Case 2: $\quad \sum_{\beta_{m},+} N^{\beta_{m}} \tilde{t}_{i}^{\beta_{m}}\left(m_{0}^{\beta_{m}}\right)^{2}<\sum_{\beta_{s}} N^{\beta_{s}}\left|\tilde{t}_{i}^{\beta_{s}}\right|\left(m_{0}^{\beta_{s}}\right)^{2}<\sum_{\beta_{m},+} N^{\beta_{m}} \tilde{t}_{i}^{\beta_{m}}\left(m_{0}^{\beta_{m}}\right)^{2}+T_{i}$.

In this case we are in a special case of (3.51) with $h_{1}=1, h_{2} \leq 1$ and

$$
\sum_{\beta_{m},+} N^{\beta_{m}} \tilde{t}_{i}^{\beta_{m}}\left(m_{0}^{\beta_{m}}\right)^{2}=h_{2} \sum_{\beta_{s}} N^{\beta_{s}}\left|\tilde{t}_{i}^{\beta_{s}}\right|\left(m_{0}^{\beta_{s}}\right)^{2} \quad\left(1-h_{2}\right) \sum_{\beta_{s}} N^{\beta_{s}}\left|\tilde{t}_{i}^{\beta_{s}}\right|\left(m_{0}^{\beta_{s}}\right)^{2}<T_{i}
$$

Analogous to (3.61) of Case 1, we obtain

$$
\begin{aligned}
T_{0} & \geq \sum_{\beta_{m},+}\left|R^{\beta_{m}}\right|-\sum_{\beta_{s}} S^{\beta_{s}}=\sum_{\beta_{m},+}\left|R^{\beta_{m}}\right|-h_{2} \sum_{\beta_{s}} S^{\beta_{s}}-\left(1-h_{2}\right) \sum_{\beta_{s}} S^{\beta_{s}} \\
& \geq \frac{1}{6} \min \left(x_{\{i, k\}}\right) \sum_{\beta_{m},+} N^{\beta_{m}} \tilde{t}_{i}^{\beta_{m}}\left(m_{0}^{\beta_{m}}\right)^{2}-\left(1-h_{2}\right) \sum_{\beta_{s}} S^{\beta_{s}} .
\end{aligned}
$$

We digress to consider the following inequality:

$$
\begin{aligned}
& \sum_{i}^{p} \sum_{\beta_{s}} N^{\beta_{s}}{\tilde{t_{i}}}^{\beta_{s}}\left(m_{0}^{\beta_{s}}\right)^{2}\left(\frac{b_{i}}{2}+\frac{j_{i}}{j_{0}}\right)=\sum_{\beta_{s}} \sum_{i}^{p} N^{\beta_{s}}\left(t_{i}^{\beta_{s}}+\mathcal{K}_{00 i}\right)\left(m_{0}^{\beta_{s}}\right)^{2}\left(\frac{b_{i}}{2}+\frac{j_{i}}{j_{0}}\right) \\
& \geq \sum_{\beta_{s}} \sum_{i}^{p} N^{\beta_{s}} t_{i}^{\beta_{s}}\left(m_{0}^{\beta_{s}}\right)^{2}\left(\frac{b_{i}}{2}+\frac{j_{i}}{j_{0}}\right) \geq \sum_{\beta} \sum_{i}^{p} N^{\beta} t_{i}^{\beta}\left(m_{0}^{\beta}\right)^{2}\left(\frac{b_{i}}{2}+\frac{j_{i}}{j_{0}}\right) \geq \sum_{i}^{p}\left(-T_{i}\right)\left(\frac{b_{i}}{2}+\frac{j_{i}}{j_{0}}\right)
\end{aligned}
$$

where in the first equality we used (3.11), in the second inequality, we extended the sum across $\beta_{s}$ to the sum across all $\beta$ because each summand is negative by (3.22), and in the last inequality we used (3.21). Comparing coefficients of $\frac{b_{i}}{2}+\frac{j_{i}}{j_{0}}$ between the first and last term in (3.65), we note that there has to exist an index $k$ such that

$$
T_{k} \geq \sum_{\beta_{s}} N^{\beta_{s}}\left|{\tilde{t_{k}}}^{\beta_{s}}\right|\left(m_{0}^{\beta_{s}}\right)^{2} \geq\left|\tilde{t_{k}} \beta^{\beta_{s}}\right|
$$

\footnotetext{
${ }^{16}$ Indeed, since a non-zero $\tilde{t}_{k}^{\beta_{m}}$ is at least a third of an integer, we only have to argue that a $k$ with a non-zero $\tilde{t}_{k}^{\beta_{m}}$ exists. But this is true since otherwise $C\left(\frac{b}{2}+\frac{j}{j_{0}}, b+\frac{m^{\beta_{m}}}{m_{0}^{\beta_{m}}}\right)=\frac{1}{2} \sum_{i=1}^{p} \tilde{t}_{i}^{\beta_{m}}\left(\frac{b_{i}}{2}+\frac{j_{i}}{j_{0}}\right) \geq 0$, which by lemma 1 implies that this brane would be a special, not a mixed brane.
} 
If the index $i$ for which we want to bound $t_{i}^{\beta}$ coincides with such an index $k$, we have an obvious bound on $t_{i}^{\beta}$

$$
T_{i} \geq \sum_{\beta_{s}} N^{\beta_{s}}\left|\tilde{t}_{i}^{\beta_{s}}\right|\left(m_{0}^{\beta_{s}}\right)^{2}>\sum_{\beta_{m},+} N^{\beta_{m}} \tilde{t}_{i}^{\beta_{m}}\left(m_{0}^{\beta_{m}}\right)^{2}>t_{i}^{\beta}
$$

where in the first inequality, we used (3.66) with $k=i$, and in the second inequality we used the assumption that $\sum_{\beta_{s}} N^{\beta_{s}}\left|\tilde{t}_{i}^{\beta_{s}}\right|\left(m_{0}^{\beta_{s}}\right)^{2}>\sum_{\beta_{m},+} N^{\beta_{m}} \tilde{t}_{i}^{\beta_{m}}\left(m_{0}^{\beta_{m}}\right)^{2}$.

Thus we only need to consider $i \neq k$ with $k$ satisfying (3.66). Then, the last term on the second line of (3.64) becomes

$$
\begin{aligned}
& \left(1-h_{2}\right) \sum_{\beta_{s}} S^{\beta_{s}} \leq\left(1-h_{2}\right) \sum_{\beta_{s}} N^{\beta_{s}} \frac{1}{2} x_{\{i, k\}}\left|\tilde{t}_{i}^{\beta_{s}}\right|\left(m_{0}^{\beta_{s}}\right)^{2}\left|\tilde{t}_{k}^{\beta_{s}}\right| \\
& \leq \frac{1}{2} x_{\{i, k\}} \underbrace{\left(1-h_{2}\right) \sum_{\beta_{s}} N^{\beta_{s}}\left|\tilde{t}_{i}^{\beta_{s}}\right|\left(m_{0}^{\beta_{s}}\right)^{2}}_{<T_{i}} \cdot T_{k}<\frac{1}{2} \max \left(x_{\{i, k\}}\right) \cdot T_{i} \cdot T_{k}
\end{aligned}
$$

where in the first inequality we plugged in the definition (3.49) of $S^{\beta_{s}}$ and picked the pair $\{i, k\}$ such that $i$ is the index for which we want to show boundedness for $t_{i}^{\beta}, k$ is the index such that (3.66) is satisfied and dropped the negative $M$-matrix term. In the second inequality we used (3.66) for $\tilde{t}_{k}^{\beta_{s}}$, as well as the second inequality in (3.63). Combining (3.64) and (3.68), we obtain

$$
T_{0}>\sum_{\beta_{m},+}\left|R^{\beta_{m}}\right|-\sum_{\beta_{s}} S^{\beta_{s}} \geq \frac{1}{6} \min \left(x_{\{i, k\}}\right) \sum_{\beta_{m},+} N^{\beta_{m}} \tilde{t}_{i}^{\beta_{m}}\left(m_{0}^{\beta_{m}}\right)^{2}-\frac{1}{2} \max \left(x_{\{i, k\}}\right) T_{i} \cdot \underbrace{T_{k}}_{\leq \max \left(T_{l}\right)}
$$

and arrive at the final bound

$$
t_{i}^{\beta}<\sum_{\beta_{m},+} N^{\beta_{m}} \tilde{t}_{i}^{\beta_{m}}\left(m_{0}^{\beta_{m}}\right)^{2}<\frac{6 T_{0}+3 T_{i} \cdot \max \left(x_{\{i, k\}}\right) \cdot \max \left(T_{l}\right)}{\min \left(x_{\{i, k\}}\right)} .
$$

\subsubsection{Bounds on $n_{I}^{D 5}$}

In this section, we employ the results from the previous section 3.3.1 to derive bounds on the numbers $n_{I}^{\mathrm{D} 5}$ of D5-branes. These bounds are formulated in two theorems.

Theorem 3. For all $i$ we have the following bound on $n_{i}^{\text {D5 }}$ :

$$
n_{i}^{D 5}<\frac{6 T_{0}}{\min \left(x_{\{i, k\}}\right)}+T_{i}
$$

where the minimum is taken over all pairs $\{i, k\}$ of indices of Kähler cone generators in the subcone, but can also be taken across the entire Kähler cone. 
Proof. From (3.3), we obtain

$n_{i}^{D 5}=\sum_{\beta} N^{\beta} t_{i}^{\beta}\left(m_{0}^{\beta}\right)^{2}+T_{i}<\sum_{\beta} N^{\beta} t_{i}^{\beta}\left(m_{0}^{\beta}\right)^{2}+T_{i}+\sum_{\beta} N^{\beta} \mathcal{K}_{00 i}\left(m_{0}^{\beta}\right)^{2}=\sum_{\beta} N^{\beta} \tilde{t}_{i}^{\beta}\left(m_{0}^{\beta}\right)^{2}+T_{i}$,

where in the last equality we used (3.11). If $\sum_{\beta} N^{\beta} \tilde{t}_{i}^{\beta}\left(m_{0}^{\beta}\right)^{2} \leq 0$, then we have the obvious bound $n_{i}^{D 5}<T_{i}$. Conversely if $0<\sum_{\beta} N^{\beta} \tilde{t}_{i}^{\beta}\left(m_{0}^{\beta}\right)^{2}$, we have

$$
0<\sum_{\beta} N^{\beta} \tilde{t}_{i}^{\beta}\left(m_{0}^{\beta}\right)^{2} \leq \sum_{\beta_{m},+} N^{\beta_{m}} \tilde{t}_{i}^{\beta_{m}}\left(m_{0}^{\beta_{m}}\right)^{2}-\sum_{\beta_{s}} N^{\beta_{s}}\left|\tilde{t}_{i}^{\beta_{s}}\right|\left(m_{0}^{\beta_{s}}\right)^{2}
$$

where we dropped negative terms in the last inequality. Thus, we are in case 1 in the proof of theorem 2, i.e. $\sum_{\beta_{s}} N^{\beta_{s}}\left|\tilde{t}_{i}^{\beta_{s}}\right|\left(m_{0}^{\beta_{s}}\right)^{2} \leq \sum_{\beta_{m},+} N^{\beta_{m}} \tilde{t}_{i}^{\beta_{m}}\left(m_{0}^{\beta_{m}}\right)^{2}$, and can use results derived previously for that case. Using the fraction $h_{1}$ defined in (3.60), (3.73) becomes

$$
\sum_{\beta} N^{\beta} \tilde{t}_{i}^{\beta}\left(m_{0}^{\beta}\right)^{2} \leq \sum_{\beta_{m},+} N^{\beta_{m}} \tilde{t}_{i}^{\beta_{m}}\left(m_{0}^{\beta_{m}}\right)^{2}-\sum_{\beta_{s}} N^{\beta_{s}}\left|\tilde{t}_{i}^{\beta_{s}}\right|\left(m_{0}^{\beta_{s}}\right)^{2}=\left(1-h_{1}\right) \sum_{\beta_{m},+} N^{\beta_{m}} \tilde{t}_{i}^{\beta_{m}}\left(m_{0}^{\beta_{m}}\right)^{2} .
$$

By the third line of (3.61), we obtain

$$
\begin{aligned}
T_{0} & \geq\left(1-h_{1}\right) \sum_{\beta_{m},+} N^{\beta_{m}} \frac{1}{2} x^{\{i, k\}} \tilde{t}_{i}^{\beta_{m}} \underbrace{\left|\tilde{t}_{k}^{\beta_{m}}\right|}_{\geq 1 / 3} \mid\left(m_{0}^{\beta_{m}}\right)^{2}+\frac{1}{6} \min \left(x_{\{i, k\}}\right) h_{1} \sum_{\beta_{m},+} N^{\beta_{m}} \tilde{t}_{i}^{\beta_{m}}\left(m_{0}^{\beta_{m}}\right)^{2} \\
& \geq \frac{1}{6} \min \left(x_{\{i, k\}}\right)\left(1-h_{1}\right) \sum_{\beta_{m},+} N^{\beta_{m}} \tilde{t}_{i}^{\beta_{m}}\left(m_{0}^{\beta_{m}}\right)^{2}
\end{aligned}
$$

by dropping the second term on the r.h.s. of the first line. By rearranging and combining with (3.74), we arrive at

$$
\sum_{\beta} N^{\beta} \tilde{t}_{i}^{\beta}\left(m_{0}^{\beta}\right)^{2} \leq(1-h) \sum_{\beta_{m},+} N^{\beta_{m}} \tilde{t}_{i}^{\beta_{m}}\left(m_{0}^{\beta_{m}}\right)^{2} \leq \frac{6 T_{0}}{\min \left(x_{\{i, k\}}\right)},
$$

which in combination with (3.72) gives the desired bound (3.71).

Remark 3. We note also, that the first inequality of (3.8) forbids $\left(n_{i}^{D 5}-T_{i}\right) \geq 0$ for all $i$, i.e. although each $n_{i}^{\mathrm{D} 5}$ is bounded above by (3.71), together they are further constrained by this condition.

Theorem 4. We have the following bound on $n_{0}^{\mathrm{D} 5}$ :

$$
n_{0}^{D 5} \leq \frac{1}{2} \max \left(x_{\{i, k\}}\right) \cdot \min \left(T_{i}\right) \cdot \max \left(T_{i}\right)+T_{0},
$$

where the minimum and maximum is taken over all pairs $\{i, k\}$ of indices of Kähler cone generators in the subcone. The maximum can also be taken across the entire Kähler cone.

Proof. Using (3.10), we obtain

$$
n_{0}^{D 5}=\sum_{\beta} N^{\beta}\left[\frac{1}{2} x_{\{i, k\}} \tilde{t}_{i}^{\beta} \tilde{t}_{k}^{\beta}-M_{\{i, k\}}\left(b+\frac{m^{\beta}}{m_{0}^{\beta}}, b+\frac{m^{\beta}}{m_{0}^{\beta}}\right)\right]\left(m_{0}^{\beta}\right)^{2}+\sum_{\gamma} N^{\gamma} C\left(m^{\gamma}, m^{\gamma}\right)+T_{0}
$$




$$
\leq \sum_{\beta_{s}} S^{\beta_{s}}-\sum_{\beta_{m},+}\left|R^{\beta_{m}}\right|+T_{0}
$$

where we dropped some negative contributions of the first term on the r.h.s. of the first line as well the negative $\gamma$-brane contribution and used $S^{\beta_{s}}, R^{\beta_{m}}$ as defined in (3.49), (3.48), respectively. We see that the coarsest bound on $n_{0}^{D 5}$ occurs when $\sum_{\beta_{s}} S^{\beta_{s}}-\sum_{\beta_{m},+}\left|R^{\beta_{m}}\right|$ is maximized. By (3.61), since its last line is positive, this expression is always negative in case 1 of theorem 2. To maximize it, we look at case 2 of theorem 2. Starting from (3.64) in case 2 of theorem 2 , we obtain

$$
\begin{aligned}
& \sum_{\beta_{m},+}\left|R^{\beta_{m}}\right|-\sum_{\beta_{s}} S^{\beta_{s}} \geq \frac{1}{6} \min \left(x_{\{i, k\}}\right) \sum_{\beta_{m},+} N^{\beta_{m}} \tilde{t}_{i}^{\beta_{m}}\left(m_{0}^{\beta_{m}}\right)^{2}-\left(1-h_{2}\right) \sum_{\beta_{s}} S^{\beta_{s}} \\
& \geq-\left(1-h_{2}\right) \sum_{\beta_{s}} S^{\beta_{s}} \geq-\left(1-h_{2}\right) \sum_{\beta_{s}} N^{\beta_{s}} \frac{1}{2} x_{\{i, k\}}\left|\tilde{t}_{i}^{\beta_{s}}\right|\left(m_{0}^{\beta_{s}}\right)^{2}\left|\tilde{t}_{k}^{\beta_{s}}\right| \\
& \geq-\frac{1}{2} \max \left(x_{\{i, k\}}\right) \underbrace{\left(1-h_{2}\right) \sum_{\beta_{s}} N^{\beta_{s}}\left|\tilde{t}_{i}^{\beta_{s}}\right|\left(m_{0}^{\beta_{s}}\right)^{2}}_{<T_{i}} \cdot T_{k}>-\frac{1}{2} \max \left(x_{\{i, k\}}\right) \cdot T_{i} \cdot T_{k} \\
& \geq-\frac{1}{2} \max \left(x_{\{i, k\}}\right) \cdot \min \left(T_{l}\right) \cdot \max \left(T_{l}\right),
\end{aligned}
$$

where in the second inequality, we dropped the positive first term. In the third inequality, we plugged in the definition (3.49) of $S^{\beta_{s}}$ and picked the pair $\{i, k\}$ such that $k$ is an index so that (3.66) is satisfied, and $i$ is the particular index such that $T_{i}=\min \left(T_{l}\right)$ if this $i \neq k$. If $i=k$, pick any other index as $i$, and drop the $M$-matrix term. In the fourth inequality we used the second inequality in (3.63). In the last inequality, we note that if we have used the first way of choosing the pair $\{i, k\}$, then $T_{i}=\min \left(T_{l}\right)$ and $T_{k} \leq \max \left(T_{l}\right)$; if we have used the second way of choosing the pair $\{i, k\}$, then $T_{i} \leq \max \left(T_{l}\right)$ and $T_{k}=\min \left(T_{l}\right)$. Combining this result with (3.78), we get the desired bound (3.77) on $n_{0}^{D 5}$.

\subsubsection{Bounds on $\gamma$-branes}

Finally, we derive a bound on the number of $\gamma$-brane configurations, i.e. we bound the flux quanta $m^{\gamma}$.

The contribution of $\gamma$-branes to the $0^{\text {th }}$-tadpole is fixed by (3.10) as

$$
-\sum_{\gamma} N^{\gamma} C\left(m^{\gamma}, m^{\gamma}\right)=T_{0}-n_{0}^{\mathrm{D} 5}+\sum_{\beta} N^{\beta}\left[\frac{1}{2} x_{\{i, k\}} \tilde{t}_{i}^{\beta} \tilde{t}_{k}^{\beta}-M_{\{i, k\}}\left(b+\frac{m^{\beta}}{m_{0}^{\beta}}, b+\frac{m^{\beta}}{m_{0}^{\beta}}\right)\right]\left(m_{0}^{\beta}\right)^{2} .
$$

As by proposition 1, the l.h.s. of this equation is positive, a solution to it only exists if the right hand side is also positive. Thus, this is the equation of an ellipsoid and the vector $m^{\gamma}$ of discrete flux quanta is given by the finite number of integral points on this ellipsoid. We denote the positive r.h.s. of (3.80) by $r^{2}$ with $r \in \mathbb{R}$.

Consequently, the question of boundedness of $m^{\gamma}$ translates into showing boundedness of $r^{2}$. By (3.80) we have

$$
r^{2}=-\sum_{\gamma} N^{\gamma} C\left(m^{\gamma}, m^{\gamma}\right)=T_{0}-n_{0}^{\mathrm{D} 5}+\sum_{\beta} N^{\beta}\left[\frac{1}{2} x_{\{i, k\}} \tilde{t}_{i}^{\beta} \tilde{t}_{k}^{\beta}-M_{\{i, k\}}\left(b+\frac{m^{\beta}}{m_{0}^{\beta}}, b+\frac{m^{\beta}}{m_{0}^{\beta}}\right)\right]\left(m_{0}^{\beta}\right)^{2}
$$




$$
\leq T_{0}+\sum_{\beta_{s}} S^{\beta_{s}}-\sum_{\beta_{m},+}\left|R^{\beta_{m}}\right| \leq T_{0}+\frac{1}{2} \max \left(x_{\{i, k\}}\right) \cdot \min \left(T_{i}\right) \cdot \max \left(T_{i}\right),
$$

where we set $n_{0}^{\mathrm{D} 5}=0$ and dropped some negative terms in the sum over $\beta$ to obtain the second line and used (3.79) for the last inequality.

This argument and also proposition 1 require that the matrix $C$ is of negative signature $(0, n)$ when restricted to the subspace of vectors obeying (3.32). As we have argued before, for the bases $B=\mathbb{F}_{k}, d P_{n}, n>1$ and the toric surfaces the matrix $C$ is of Minkowski signature and the vector $\frac{b}{2}+\frac{j}{j_{0}}$ is time-like. Thus, the above argument applies.

\section{Conclusions}

We have studied Type IIB compactifications on smooth Calabi-Yau elliptic fibrations over almost Fano twofold bases $B$ with magnetized D9-branes and D5-branes. We have proven that the tadpole cancellation and SUSY conditions imply that there are only finitely many such configurations. We have derived explicit and calculable bounds on all flux quanta (table 1, theorem 2, section 3.3.3) as well as the number of D5-branes (theorem 3, theorem 4), which are independent on the continuous moduli of the compactification, in particular the Kähler moduli, as long as the supergravity approximation is valid.

The presented proof applies for any geometry that meets the geometric conditions listed at the beginning of section 3.3. We have shown explicitly in section 2.3 and appendix A that these geometric conditions are obeyed for the twofold bases $B$ given by the Hirzebruch surfaces $\mathbf{F}_{k}, k=0,1,2$, the generic del Pezzos $d P_{n}, n=0, \ldots, 8$ as well all toric varieties associated to the 16 reflexive two-dimensional polytopes. This in particular required showing the positive semi-definiteness of the matrices $M_{\{i, k\}}$ defined in (3.9). To this end we studied the Kähler cones of the generic $d P_{n}$ and explicitly constructed their Kähler cone generators, which are listed in table 3 and reveal useful geometric properties of these Kähler cones.

Physically, we have proven that there exists a finite number of four-dimensional $\mathcal{N}=1$ supergravity theories realized by these compactifications. Most notably, there arise only finitely many gauge sectors in these theories with finitely many different chiral spectra. The details of these gauge sectors are determined by the bounded number of branes in a stack and the bounded magnetic flux quanta. Concretely, this means that the ranks of the gauge groups are bounded, that only certain matter representations with certain chiral indices exist (which is always true in weakly coupled Type IIB) and that for fixed gauge group there exist only a finite set of possible multiplicities for the matter fields. These finiteness properties, and more broadly similar results elsewhere in the landscape, are particularly interesting when contrasted to generic quantum field theories.

While we have shown finiteness of these compactifications and provided explicit bounds, we have not explicitly constructed all of these compactifications. It would be interesting to systematically construct this finite set of configurations and extract generic features of the four-dimensional effective theories in this corner of the landscape. In addition, we have not systematically explored the bases $B$ for which the proof applies, i.e. there may exist additional algebraic surfaces satisfying the geometric conditions of section 3.3. 
Other points of interest would be to determine whether a simple modification of our proof exists for blow-ups of singular elliptic fibrations or elliptically fibered Calabi-Yau manifolds which do not satisfy the supergravity approximation; in the latter case the supersymmetry conditions receive corrections of various types. Of most interest would be to find a general proof for a general Calabi-Yau threefold $X$. It seems plausible that there are even more general proof techniques which utilize SUSY and tadpole cancellation conditions to prove finiteness for a general $X$. For example, some of the arguments in the proof presented here, e.g. the ones used to eliminate the dependence of the SUSY conditions (2.4) on the Kähler moduli, should still apply for general Calabi-Yau manifolds $X$. In addition, string dualities of the considered Type IIB configurations extend our finiteness proof to the dual theories, for example to the heterotic string on certain elliptic fibrations with specific vector bundles and to F-theory on certain elliptic $K 3$-fibered fourfolds. It is very important to work out the details of the duality maps and the analogs of the bounds we found in the dual theories.

The presented proof is based on tadpole and supersymmetry conditions at weak coupling and large volume of $X$. It is crucial for a better understanding of the string landscape to understand string consistency conditions away from large volume and weak coupling. This requires the understanding of perturbative and non-perturbative corrections ${ }^{17}$ both in $\alpha^{\prime}$ and in $g_{S}$; for example, the supersymmetry conditions receive $\alpha^{\prime}$-corrections from worldsheet instantons. Avenues towards a better understanding might be provided by applications of $\mathcal{N}=1$ mirror symmetry, i.e. mirror symmetry, and $S$-duality.

It is particularly interesting that the finiteness results we have proven and similar results elsewhere in the landscape do not have known analogs in generic quantum field theories. Such differences are one of the hallmarks of string compactifications, and it seems reasonable to expect that similar finiteness results can be proven for even the most general string compactifications, in particular those at small volume and strong coupling. This would have profound implications for our picture of the landscape: while it is larger than originally thought, our results provide further evidence that it may, in fact, be finite.

\section{Acknowledgments}

We thank Mike Douglas, Antonella Grassi, Albrecht Klemm, Dave Morrison, Hernan Piragua and Wati Taylor for useful conversations and correspondence. This research is supported in part supported by the DOE grant DE-SC0007901 (M.C. and D.K.), Dean's Funds for Faculty Working Group (M.C. and D.K.), the Fay R. and Eugene L. Langberg Endowed Chair (M.C.), the Slovenian Research Agency (ARRS) (M.C.) and the NSF grant PHY11-25915 (J.H.). J.H. thanks J.L. Halverson for her encouragement.

\section{A Kähler cones of del Pezzo surfaces \& their $M_{\{i, k\}}$-matrices}

In this appendix we discuss in detail the structure of the Kähler cone of the del Pezzo surfaces $d P_{n}$ for $n \leq 8$. We are interested in the extremal rays, i.e. the generators, of these

\footnotetext{
${ }^{17}$ See [64-67] for recent computations of corrections to $\mathcal{N}=1$ couplings in M-/F-theory compactifications.
} 
in general non-simplicial cones, and the existence of coverings of these cones by simplicial subcones so that conditions (1)-(3) listed at the beginning of section 3.3 are obeyed.

First, we expand the Kähler cone generators $D_{i}$ of $d P_{n}$ in the basis $(2.20)$ of $H^{2}\left(d P_{n}, \mathbb{Z}\right)$

$$
D_{i}=\left(v_{i}\right)^{1} H+\sum_{j=1}^{n}\left(v_{i}\right)^{j} E_{j},
$$

which maps every $D_{i}$ to a vector $v_{i}$ in $\mathbb{Z}^{n+1}$. With this definition, we obtain the matrices (3.9) in this basis as

$$
M_{\{i, k\}}=\eta \cdot\left[x_{\{i, k\}}\left(v_{i} \cdot v_{k}^{T}+v_{k} \cdot v_{i}^{T}\right)-\eta\right] \cdot \eta,
$$

where $i \neq k, v^{T}$ denotes the transpose of a vector, ' $'$ denotes the matrix product and $\eta=\operatorname{diag}(1,-1, \ldots,-1)$ is the standard Minkowski matrix in $n+1$ dimensions. We note that in order to check positive semi-definiteness of the matrices in (A.2), it suffices to prove it for the matrices $\eta \cdot M_{\{i, k\}} \cdot \eta$, which is the matrix in the square brackets in (A.2).

Next, we need the explicit form for the Kähler generators of $d P_{n}$. We present these by listing the corresponding vectors $v_{i}$ defined via (A.1). We explicitly solve (2.25) over the integers to obtain the Kähler cone generators. For the simplicial cases $d P_{0}, d P_{1}, d P_{2}$ we obtain (2.26) as discussed earlier. In the non-simplicial cases $d P_{n}, n>2$, we summarize the generators in table 3 .

Here, the second column contains the schematic form of the vectors $v_{i}$, with each row containing all vectors of the same particular form. In each row, the explicit expressions for the $v_{i}$ are obtained by inserting the values listed in the third column for the place holder variables in the entries of $v_{i}$ in that row and by permuting the underlined entries of the vector $v_{i}$. The number of different vectors in each row is given in the fourth column, where the two factors are given by the number of elements in the list in the third column and the number of permutations of the entries, respectively. The fifth column contains a list of the Minkowski length of all vectors in a given row. We note that this column precisely contains the self-intersection of the curves associated to the $D_{i}$. All are either 0 or 1 and it can be checked that the intersections of the $v_{i}$ with $c_{1}\left(d P_{n}\right)=3 H-\sum_{i} E_{i} \equiv(3,-1, \ldots,-1)$ are precisely 2 or 3 , respectively, as required by $(2.25)$.

For example, in the second row of table 3 , all vectors $v_{i}$ are of the form $v_{i}=(a, b, b, b)$ by the second column. By the third column, there are two different vectors of this type, namely $v_{1}=(2,-1,-1,-1)$ and $v_{2}=(1,0,0,0)$. Thus, there are precisely 2 vectors as indicated in the fourth column and the Minkowski length of the two vectors is 1, 1 , respectively, as in the last column of the second row.

We note that the Kähler cone generators and their grouping as in table 3 can be understood by representation theory, recalling that the Weyl group naturally acts on $H_{2}\left(d P_{n}, \mathbb{Z}\right)$. For instance the Kähler cone generators of $d P_{n}, n=2, \ldots, 6$ form the representations 3, $(\overline{\mathbf{3}} \otimes \mathbf{1}) \oplus(\mathbf{1} \otimes \mathbf{2}), \mathbf{5} \oplus \overline{\mathbf{5}}, \mathbf{1 6} \oplus \mathbf{1 0}$ and $\mathbf{7 8} \oplus \mathbf{2 7}$ under the corresponding groups $A_{1}, A_{2} \times A_{1}$, $A_{4}, D_{5}$ and $E_{6}$, respectively. Here the first representation in all direct sums is formed by all generators with Minkowski length 1 and the second one is formed by generators with Minkowski length 0 . These results can be worked out explicitly by computing the Dynkin 


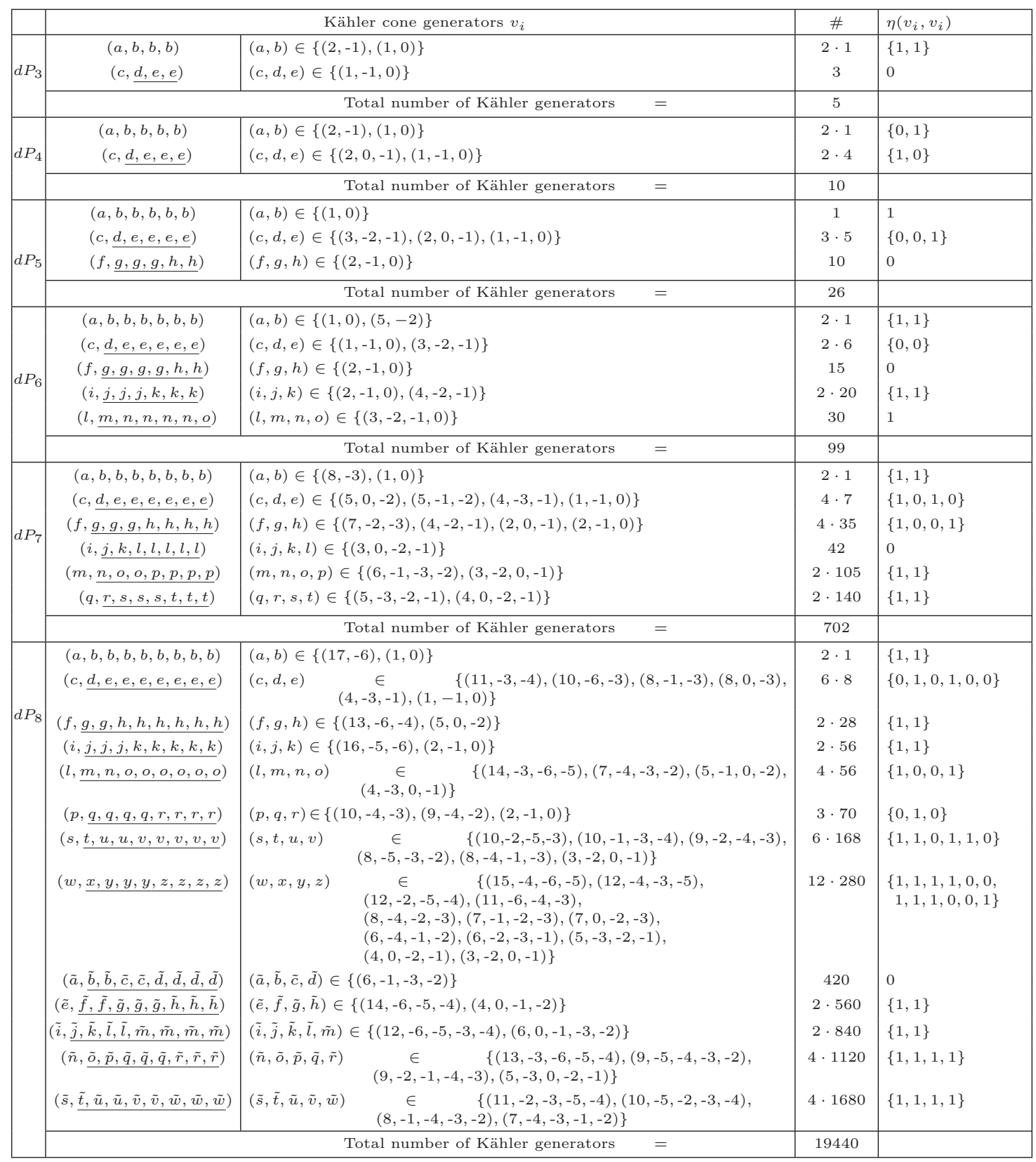

Table 3. Kähler cone generators for $d P_{n}$. The underlined entries of the $v_{i}$ are permuted.

labels of the generators in table 3 for the canonical roots $\alpha_{i}$, which are the -2 -curves in $H_{2}\left(d P_{n}, \mathbb{Z}\right)$ orthogonal to $c_{1}\left(d P_{n}\right)$. Thus, the zero weight vector is identified with $c_{1}\left(d P_{n}\right)$. For $d P_{7}$ only the union of the generators of the Kähler and Mori cone have a representation theoretical decomposition as $\mathbf{9 1 2} \oplus \mathbf{1 3 3}$ (some of the weights of the $\mathbf{9 1 2}$ have higher multiplicities yielding only 576 different weights), where the first representation contains the length 1 and the second one the length 0 Kähler cone generators.

Next, we make one important observation. As one can check explicitly from table 3 and (2.26), for every del Pezzo $d P_{n}$ with $n>1$, the first Chern class $c_{1}\left(d P_{n}\right) \equiv$ 
$(3,-1, \ldots,-1)$ is proportional to the sum of all Kähler cone generators $v_{i}$

$$
c_{1}\left(d P_{n}\right) \equiv(3,-1, \ldots,-1)=\frac{1}{A_{n} \cdot N} \sum_{i=1}^{N} v_{i}
$$

where $N$ denotes the total number of Kähler cone generators of $d P_{n}$, cf. table 3 . The positive proportionality factor $A_{n}$ depends on $n$ and reads

$$
A_{3}=\frac{2}{5}, \quad A_{4}=\frac{1}{2}, \quad A_{5}=\frac{17}{26}, \quad A_{6}=\frac{10}{11}, \quad A_{7}=\frac{55}{39}, \quad A_{8}=\frac{26}{9}
$$

for $d P_{3}, d P_{4}, d P_{5}, d P_{6}, d P_{7}$ and $d P_{8}$, respectively. This means that $c_{1}\left(d P_{n}\right)$ is in the center of the Kähler cone of all del Pezzo surfaces with $n>1$.

This implies that we can find a cover of the Kähler cone by simplicial subcones so that properties (1)-(3) at the beginning of section 3.3 are satisfied. We present two such covers:

Cover 1: intersect the Kähler cone with a hyperplane that is normal to $c_{1}\left(d P_{n}\right)$ and passes through $c_{1}\left(d P_{n}\right)$. This yields an $n$-dimensional polytope with vertices corresponding to the generators of the Kähler cone. Triangulate this polytope with star being $c_{1}\left(d P_{n}\right)$. This triangulation induces a decomposition of the Kähler cone into simplicial subcones. As the generators of one simplicial subcone, take $c_{1}\left(d P_{n}\right)$ and those generators $v_{i}$ of the Kähler cone that go through the vertices of an $n$-dimensional cone of the triangulated polytope.

In this covering of the Kähler cone, properties (2) and (3) are satisfied: we obviously have $b_{i}$ all positive, because $c_{1}\left(d P_{n}\right)$ is one of the generators in every simplicial subcone. From (2.10) we get $b_{i}=0$ for all $D_{i} \neq c_{1}\left(d P_{n}\right)$ and $b_{K}=1$, where $K$ denotes the index such that $D_{K}=c_{1}\left(d P_{n}\right)$. In addition, we have $C_{K K}=\mathcal{K}_{000}=9-n$ and $C_{i K}=\mathcal{K}_{00 i}=2,3$ for $i \neq K$ by (2.7) and (2.27) and $T_{K}=12 \int_{B} c_{1}^{2}=12 \mathcal{K}_{000}=12(9-n)$ by $(2.13)$ and (2.27). We discuss why property (1) is satisfied later.

Cover 2: although the above cover 1 obeys all the required properties listed at the beginning of section 3.3, it slightly increases the bounds because it increases $\max \left(T_{i}\right)$ for $n \leq 6$ in which case $\max \left(T_{i}\right)=T_{K}=12(9-n)$ is larger than the $T_{i}$ found in (2.28).

Thus, we provide the following alternative cover which exists if the Kähler cone is sufficiently symmetric, in addition to $c_{1}\left(d P_{n}\right)$ being its center. Take a vertex of the polytope constructed in cover 1. Construct the line through that vertex and the star, i.e. $c_{1}\left(d P_{n}\right)$. This line has to intersect the boundary of the polytope at another point. This point lies on a certain facet of this polytope. Take the vertices of this facet together with the original vertex we have started with to define a simplicial subcone of the Kähler cone. Notice that this subcone contains $c_{1}\left(d P_{n}\right)$ and the cone formed by the vertices of this facet and $c_{1}\left(d P_{n}\right)$, i.e. a subcone in cover 1 . Repeat this procedure for all vertices of the polytope. If the Kähler cone is sufficiently symmetric, each facet will be hit exactly once. Thus, each subcone in cover 1 is contained in a corresponding subcone defined in this way. Consequently, since cover 1 covers the Kähler cone completely, so does cover 2 .

This cover also satisfies conditions (1)-(3) at the beginning of section 3.3. We again leave the discussion of condition (1) for later. Conditions (2) and (3) are satisfied since $c_{1}\left(d P_{n}\right)$ is contained in each subcone, which implies $b_{i} \geq 0$ for all $i$, and by $(2.27)$ all $\mathcal{K}_{00 i}$ 
are positive integers. In addition, the advantage of this cover is that all generators of all simplicial subcones are generators of the Kähler cone. Thus in all bounds derived in this work, we have that $\max \left(T_{i}\right)$ is given precisely by (2.28). Given the fact that the generators of the Kähler cone sit in representations of Lie algebras, which implies that the Kähler cone is symmetric, and that $c_{1}\left(d P_{n}\right)$ lies in its center, we expected this cover 2 to exist.

Finally, we discuss why condition (1), i.e. the positive semi-definiteness of the matrices $M_{\{i, k\}}$ in (3.9), is satisfied in both Cover 1 and Cover 2. We notice the following fact: for both covers, in order to show that the matrices (3.9) are positive semi-definite, we only have to prove that these matrices written in the form (A.2) are positive semi-definite for all possible choices of two vectors $v_{i}, v_{j}$ of table 3 . This is clear for Cover 2 , because the generators of all simplicial subcones are generators of the Kähler cone. For Cover 1, in every simplicial subcone, all matrices $M_{\{i, j\}}$ with $i, j \neq K$ involve only the generators $v_{i}$, $v_{j}$. Thus, we only have to consider the matrices $M_{\{i, K\}}$ with $i \neq K$ (recall that we only have to show positive semi-definiteness of the matrices $M_{\{i, j\}}$ for $i \neq j$ ). For these we use

Lemma 5. In Cover 1 , let $K$ be the index corresponding to $c_{1}\left(d P_{n}\right)$, then the matrices $M_{\{i, K\}}$ for all $i \neq K$ are positive semi-definite, if all matrices $M_{\{i, j\}}$ for all pairs of generators $v_{i}, v_{j}$ of the Kähler cone are positive semi-definite.

Proof. Using the first Chern class $c_{1}\left(d P_{n}\right) \equiv(3,-1, \ldots,-1)$ and $\lambda_{j}=\frac{1}{A_{n} \cdot N}$, we obtain

$$
M_{\{i, K\}}=x_{\{i, K\}}\left(v_{i} \cdot c_{1}\left(d P_{n}\right)^{t}+c_{1}\left(d P_{n}\right) \cdot v_{i}^{t}\right)-\eta=\sum_{j=1}^{N} \lambda_{j} x_{\{i, K\}}\left(v_{i} \cdot v_{j}^{t}+v_{j} \cdot v_{i}^{t}\right)-\eta,
$$

where we used (A.3). Choose $x_{\{i, K\}}$ for every $i$ so that the following equality is satisfied

$$
\sum_{j=1}^{N} \lambda_{j} \frac{x_{\{i, K\}}}{x_{\{i, j\}}}=x_{\{i, K\}} \sum_{j=1}^{N} \frac{\lambda_{j}}{x_{\{i, j\}}}=x_{\{i, K\}} \frac{1}{A_{n}}\left\langle\frac{1}{x_{\{i, j\}}}\right\rangle_{j} \stackrel{!}{=} 1
$$

where $\left\langle\frac{1}{x_{\{i, j\}}}\right\rangle_{j}$ denotes the average of $\frac{1}{x_{\{i, j\}}}$ with $i$ kept fixed and $j$ varied over all Kähler cone generators. Then, (A.5) can be written as

$$
\begin{aligned}
M_{\{i, K\}} & =\sum_{j=1}^{N} \lambda_{j} \frac{x_{\{i, K\}}}{x_{\{i, j\}}} x_{\{i, j\}}\left(v_{i} \cdot v_{j}^{t}+v_{j} \cdot v_{i}^{t}\right)-\eta=\sum_{j=1}^{N} \lambda_{j} \frac{x_{\{i, K\}}}{x_{\{i, j\}}}\left(x_{\{i, j\}}\left(v_{i} \cdot v_{j}^{t}+v_{j} \cdot v_{i}^{t}\right)-\eta\right) \\
& =\sum_{j=1}^{N} \lambda_{j}^{\prime}\left(x_{\{i, j\}}\left(v_{i} \cdot v_{j}^{t}+v_{j} \cdot v_{i}^{t}\right)-\eta\right)=\sum_{j=1}^{N} \lambda_{j}^{\prime} M_{\{i, j\}},
\end{aligned}
$$

where we set $\lambda_{j}^{\prime}=\lambda_{j} \frac{x_{\{i, K\}}}{x_{\{i, j\}}}$. We note that $M_{\{i, K\}}$ is defined in terms of generators of the Kähler cone and $\lambda_{j}^{\prime} \geq 0$ for all $j=1, \ldots, N$. Thus, if all the $M_{\{i, j\}}$ are positive semi-definite, then $M_{\{i, K\}}$ will be automatically positive semi-definite because it is just a positive linear combination of the $M_{\{i, j\}}$ by (A.7). A positive linear combination of positive semi-definite matrices is again positive semi-definite. 
Thus, it only remains to show positive semi-definiteness of the matrices $M_{\{i, k\}}$ defined in (A.2) for any choice of two Kähler cone generators of $d P_{n}$ from table 3 . We note that the Kähler cone generators of $d P_{n}$ are obtained by permutations of the vectors in table 3 . Most of these permutations simply interchange the rows and columns of the matrices (A.2), which does not affect their eigenvalues. Thus, we only have to consider matrices (A.2) that do not differ only by such a permutation. We provide an efficient algorithm making use of this permutation symmetry to generate all matrices (A.2) with different sets of eigenvalues. Recall that to check positive-semi-definiteness for any $M_{\{i, k\}}$, it suffices to check positivesemi-definiteness for $\tilde{M}_{\{i, k\}}$, defined as

$$
\tilde{M}_{\{i, k\}}=x_{\{i, k\}}\left(v_{i} \cdot v_{k}^{T}+v_{k} \cdot v_{i}^{T}\right)-\eta .
$$

For each $\tilde{M}_{\{i, k\}}$ define $\left(v_{i}, v_{k}\right)$ as the pair of Kähler cone generators in its definition (A.8). By definition of $M_{\{i, k\}}$, we have $i \neq k$ in $\left(v_{i}, v_{k}\right)$. For each $d P_{n}$, we define an equivalence relation on the set of all pairs $\left(v_{i}, v_{k}\right)$ and show if $\left(v_{i}, v_{k}\right) \sim\left(v_{i}^{\prime}, v_{k}^{\prime}\right)$ and $x_{\{i, k\}}=$ $x_{\{i, k\}}^{\prime}$, the corresponding matrices $\tilde{M}_{\{i, k\}}$ and $\tilde{M}_{\{i, k\}}^{\prime}$ have the same sets of eigenvalues.

Definition 2. For each $d P_{n}$, let $\left\{\left(v_{i}, v_{k}\right)\right\}, i \neq k$, be the set of all pairs of its Kähler cone generators. The symmetric group $S_{n}$ of degree $n$ acts on the Kähler cone generator $v_{i} \in \mathbb{Z}^{1+n}$ by permuting its last $n$ components, cf. the second column of table 3. Define an equivalence relation $\sim$ on $\left\{\left(v_{i}, v_{k}\right)\right\}$ by $\left(v_{i}, v_{k}\right) \sim\left(v_{i}^{\prime}, v_{k}^{\prime}\right)$ if $\left(v_{i}^{\prime}, v_{k}^{\prime}\right)=\left(\sigma\left(v_{i}\right), \sigma\left(v_{k}\right)\right)$, for some $\sigma \in S_{n}$.

Lemma 6. Suppose $\left(v_{i}, v_{k}\right) \sim\left(v_{i}^{\prime}, v_{k}^{\prime}\right)$. Let $\tilde{M}_{\{i, k\}}$ and $\tilde{M}_{\{i, k\}}^{\prime}$ be the matrix defined by $\left(v_{i}, v_{k}\right)$ and $\left(v_{i}^{\prime}, v_{k}^{\prime}\right)$, respectively, with $x_{\{i, k\}}=x_{\{i, k\}}^{\prime}$, in (A.8). Then $\tilde{M}_{\{i, k\}}$ and $\tilde{M}_{\{i, k\}}^{\prime}$ have the same set of eigenvalues.

Proof. Let $\sigma \in S_{n}$ so that $\left(v_{i}^{\prime}, v_{k}^{\prime}\right)=\left(\sigma\left(v_{i}\right), \sigma\left(v_{k}\right)\right)$. Denote the permutation matrix that permutes the $j^{\text {th }}$ and $l^{\text {th }}$ rows/columns by $P_{j l}$. Since any $\sigma \in S_{n}$ can be written as a product of such permutation matrices, we can WLOG assume $\sigma=P_{j l}$. Then we have

$$
\begin{aligned}
\tilde{M}_{\{i, k\}}^{\prime} & =x_{\{i, k\}}\left(P_{j l} v_{i} v_{k}^{T} P_{j l}^{T}+P_{j l} v_{k} v_{i}^{T} P_{j l}^{T}\right)-\eta=x_{\{i, k\}}\left(P_{j l} v_{i} v_{k}^{T} P_{j l}^{T}+P_{j l} v_{k} v_{i}^{T} P_{j l}^{T}\right)-P_{j l} \eta P_{j l}^{T} \\
& =P_{j l}\left[x_{\{i, k\}}\left(v_{i} v_{k}^{T}+v_{k} v_{i}^{T}\right)-\eta\right] P_{j l}^{T}=P_{j l} \tilde{M}_{\{i, k\}} P_{j l}^{T} .
\end{aligned}
$$

This implies that the characteristic polynomials of $\tilde{M}_{\{i, k\}}$ and $\tilde{M}_{\{i, k\}}^{\prime}$ are the same,

$$
\begin{aligned}
\operatorname{det}\left(\tilde{M}_{\{i, k\}}^{\prime}-\lambda I\right) & =\operatorname{det}\left(P_{j l} \tilde{M}_{\{i, k\}} P_{j l}^{T}-\lambda P_{j l} I P_{j l}^{T}\right)=\operatorname{det}\left(P_{j l}\left(\tilde{M}_{\{i, k\}}-\lambda I\right) P_{j l}^{T}\right) \\
& =\operatorname{det}\left(P_{j l}\right) \operatorname{det}\left(\tilde{M}_{\{i, k\}}-\lambda I\right) \operatorname{det}\left(P_{j l}^{T}\right)=\operatorname{det}\left(\tilde{M}_{\{i, k\}}-\lambda I\right) .
\end{aligned}
$$

Lemma 6 shows that for each equivalence class $\left[\left(v_{i}, v_{k}\right)\right]$, we just need to pick any representative $\left(v_{i}, v_{k}\right)$ and check if there exists an $x_{\{i, k\}} \in \mathbb{Q}^{+}$such that $\left(v_{i}, v_{k}\right)$ and $x_{\{i, k\}}$ defines a positive semi-definite matrix $\tilde{M}_{\{i, k\}}$ according to (A.8). If such an $x_{\{i, k\}}$ exists, any $\tilde{M}_{\{i, k\}}^{\prime}$ with $\left(v_{i}^{\prime}, v_{k}^{\prime}\right) \sim\left(v_{i}, v_{k}\right)$ will be automatically positive semi-definite for $x_{\{i, k\}}^{\prime}=x_{\{i, k\}}$. For each $d P_{n}$, in order to find all different equivalence classes, we start by picking an arbitrary pair $\left(v_{i}, v_{k}\right)$ from table 3 and carry out the following algorithm: 
(1) Fix $v_{i}$ and only permute the entries of $v_{k}$. Indeed, if $v_{i}^{\prime}=\sigma\left(v_{i}\right), v_{k}^{\prime}=\tau\left(v_{k}\right)$, then $\left(v_{i}^{\prime}, v_{k}^{\prime}\right) \sim\left(v_{i}, \sigma^{-1} \tau\left(v_{k}\right)\right)$. Let $\tau^{\prime}=\sigma^{-1} \tau$, then we have $\left[\left(v_{i}^{\prime}, v_{k}^{\prime}\right)\right]=\left[\left(v_{i}, \tau^{\prime}\left(v_{k}\right)\right)\right]$.

(2) Only permute those entries in $v_{k}$ for which the corresponding entries in $v_{i}$ are different from each other. Permuting two entries in $v_{k}$ when the corresponding two entries in the fixed vector $v_{i}$ are the same is equivalent to the action of permuting these two entries for both vectors. Thus, the resulting pair of vectors $\left(v_{i}, v_{k}^{\prime}\right) \sim\left(v_{i}, v_{k}\right)$.

Pick a different pair $\left(w_{i}, w_{k}\right)$ of Kähler cone generators from table 3 and repeat $(1),(2)$.

For example, consider $d P_{8}$. Suppose we begin by picking $v_{i}=(a, b, b, b, b, b, b, b, b)$ and $v_{k}=(s, t, u, u, v, v, v, v, v)$ from the second column of table 3. By (1) above, we can fix $v_{i}$ and only consider permutations in the last eight entries of $v_{k}$. By (2), however, we do not need to consider any permutation in the last eight entries in $v_{k}$, because the last eight entries in the fixed vector $v_{i}$ are the same; they are all equal to $b$. Thus, there is only one equivalence class $\left[\left(v_{i}, v_{k}\right)\right]$. From the third column of table 3 , there are two sets of different values for $v_{i}=(a, b, b, b, b, b, b, b, b)$, and six sets of different values for $v_{k}=(s, t, u, u, v, v, v, v, v)$. Thus there will be $2 \cdot 6=12$ different $\tilde{M}_{\{i, k\}}^{\prime}$ matrices to check for positive semi-definiteness. Next pick a different pair of $\left(w_{i}, w_{k}\right)$ and repeat this process.

We obtain that the matrices (A.2) are positive semi-definite for any choice of two Kähler cone generators in table 3 and $x_{\{i, k\}}$ of the form

$$
x_{\{i, k\}}=\frac{1}{a} \quad \text { for } \quad a \in\{1,2, \ldots, 19\} .
$$

More precisely, for $d P_{2}$ and $d P_{3}$ all $x_{\{i, k\}}=1$, for $d P_{4}$ and $d P_{5}$ we have $x_{\{i, k\}}=1, \frac{1}{2}$, for $d P_{6}$ we have $x_{\{i, k\}}=\frac{1}{a}$ with $a \in\{1,2, \ldots, 4\}$, for $d P_{7}$ we find $x_{\{i, k\}}=\frac{1}{a}$ with $a \in\{1,2, \ldots, 7\}$ and for $d P_{8}$ all values in (A.11) are assumed.

\section{B Geometric data of almost Fano twofolds for computing explicit bounds}

In this appendix, we summarize the geometric data of Hirzebruch surfaces $\mathbb{F}_{k}, k=0,1,2$, the del Pezzo surfaces $d P_{n}, n=2, \ldots, 8$, and the toric varieties associated to the 16 reflexive polytopes that is necessary to explicitly compute the various bounds derived in this paper.

We begin with the bases $\mathbb{F}_{k}$ and $d P_{n}$. The following results in table 4 are derived employing (2.19), (2.28), the two covers of the Kähler cones of $d P_{n}$ constructed in appendix A, (A.4) and the values of $x_{\{i, k\}}$ listed below (A.11).

First, we list the maximal and minimal values of $x_{\{i, k\}}$ and $T_{i}$ for the bases $\mathbb{F}_{k}$ and $d P_{2}$ that have a simplicial Kähler cone. For the non-simplicial Kähler cones, we obtain different results for the two different covers of their Kähler cones. We note that for both cover 1 and 2 the values below (A.11) apply. Indeed, this is precisely what we get in the second and third column under cover 2. However, for cover 1, these numbers have to be multiplied by appropriate $A_{n}$ in (A.4). Indeed, by (A.6) we have $x_{\{i, K\}}=A_{n}\left(\left\langle x_{\{i, k\}}\right\rangle_{j}\right)^{-1}$. By (A.4), we have $A_{n} \leq 1$ for $n \leq 6$, i.e. the minimum value of $x_{\{i, K\}}$ is bounded by $A_{n} \cdot \min \left(x_{\{i, K\}}\right)$, but the maximum is unaffected, as indicated in the first four rows of the second and third column in table 4 under cover 1 . For $d P_{7}$ and $d P_{8}$, we have $A_{n}>1$, thus 


\begin{tabular}{|c|c|c|c|c|}
\hline & $\max \left(x_{\{i, k\}}\right)$ & $\min \left(x_{\{i, k\}}\right)$ & $\max \left(T_{i}\right)$ & $\min \left(T_{i}\right)$ \\
\hline $\mathbb{F}_{k}$ & 1 & 1 & $24+12 k$ & 24 \\
$d P_{2}$ & 1 & 1 & 36 & 24 \\
\hline & \multicolumn{4}{|c|}{ Cover 1 of Kähler cone of $d P_{n}$} \\
\hline$d P_{3}$ & 1 & $A_{3} \leq$ & 72 & 24,36 \\
$d P_{4}$ & 1 & $\frac{1}{2} A_{4} \leq$ & 60 & 24,36 \\
$d P_{5}$ & 1 & $\frac{1}{2} A_{5} \leq$ & 48 & 24,36 \\
$d P_{6}$ & 1 & $\frac{1}{4} A_{6} \leq$ & 36 & 24,36 \\
$d P_{7}$ & $A_{7} \geq$ & $\frac{1}{7}$ & 36 & 24 \\
$d P_{8}$ & $A_{8} \geq$ & $\frac{1}{19}$ & 36 & 12 \\
\hline & \multicolumn{5}{|c|}{ Cover 2 of Kähler cone of $d P_{n}$} \\
\hline$d P_{3}$ & 1 & 1 & 36 & 24,36 \\
$d P_{4}$ & 1 & $\frac{1}{2}$ & 36 & 24,36 \\
$d P_{5}$ & 1 & $\frac{1}{2}$ & 36 & 24,36 \\
$d P_{6}$ & 1 & $\frac{1}{4}$ & 36 & 24,36 \\
$d P_{7}$ & 1 & $\frac{1}{7}$ & 36 & 24,36 \\
$d P_{8}$ & 1 & $\frac{1}{19}$ & 36 & 24,36 \\
\hline
\end{tabular}

Table 4. Key geometrical data for the computation of the explicit bounds derived in the proof.

$x_{\{i, K\}} \leq A_{n} \max \left(x_{\{i, k\}}\right)=A_{n}$ and the minimum is unaffected, as displayed in the last two rows of the second and third column in table 4 for cover 1.

In addition, without knowing every simplicial subcone in the two covers explicitly, we can not determine the explicit value $\min \left(T_{i}\right)$ for both covers. Therefore, depending on the chosen subcone, employing (2.28), we either obtain 24 or 36 as indicated in the last column of table 4 . However, in the case of cover 1 we have $T_{K}=24,12$ for $d P_{7}$ and $d P_{8}$, respectively. Since by construction, the first Chern class $c_{1}\left(d P_{n}\right)$ is in every subcone, we know that $\min \left(T_{i}\right)=T_{K}=24,12$ for $d P_{7}$ and $d P_{8}$, respectively.

Finally, in table 5 we display the relevant topological data of the toric varieties constructed from the 16 reflexive two-dimensional polytopes which is relevant to our finiteness proof in section 3.3. We confirmed that the first Chern class $c_{1}(B)$ is inside the Kähler cone in all these cases, i.e. Cover 1 constructed in appendix A exists for these non-simplicial Kähler cones. As explained there, in this cover the conditions (2) and (3) listed at the beginning of section 3.3 are obeyed. We also checked that the matrices (A.2) are all positive semi-definite for $x_{\{i, k\}}$ of the form $x_{\{i, k\}}=\frac{1}{a}$ with $a \in\{1, \ldots, 6\}$, i.e. condition (1) listed in section 3.3 is also satisfied. 


\begin{tabular}{|c|c|c|c|c|}
\hline Polytope & $\int c_{2}$ & $\int c_{1}^{2}$ & $\mid$ K.C. Gens $\mid$ & List of $T_{i}=12 \int_{D_{i}} c_{1}$ \\
\hline 2 & 4 & 8 & 2 & $(24,24)$ \\
3 & 4 & 8 & 2 & $(24,36)$ \\
4 & 4 & 8 & 2 & $(24,48)$ \\
5 & 5 & 7 & 3 & $(24,24,36)$ \\
6 & 5 & 7 & 3 & $(24,36,48)$ \\
7 & 6 & 6 & 5 & $(24,24,24,36,36)$ \\
8 & 6 & 6 & 4 & $(24,36,24,48)$ \\
9 & 6 & 6 & 5 & $(24,36,24,48,36)$ \\
10 & 6 & 6 & 4 & $(24,48,76,48,24,36,36,48,48,24,36)$ \\
11 & 7 & 5 & 7 & $(24,48,36,72,48,36,24,72,48,48)$ \\
12 & 7 & 5 & 8 & $(24,24,36,48,36,48,24,72,36,48,72,48,36)$ \\
13 & 8 & 4 & 10 & $(24,24,36,72,48,36,48,36,48,36,48$, \\
14 & 8 & 4 & 13 & $72,72,72,36,48,24,36,72,48,72)$ \\
15 & 8 & 4 & 12 & \\
16 & 9 & 3 & 21 &
\end{tabular}

Table 5. Displayed are some of the relevant data for the smooth almost Fano toric surfaces obtained from fine star triangulations of the two-dimensional reflexive polytopes in figure 1 .

\section{An analytic proof of positive semi-definiteness of the $M_{\{i, k\}}$-matrices}

In this section we provide an alternative general proof of positive semi-definiteness of the $M_{\{i, k\}}$-matrices, in comparison to the numerical proof given in appendix A for specific $B$.

We recall that to check positive-semi-definiteness for any $M_{\{i, k\}}$ defined in (A.2) it suffices to check positive semi-definiteness for the matrix $\tilde{M}_{\{i, k\}}$ defined in (A.8). The advantage of the following general proof is that it predicts a precise value of $x_{\{i, k\}}$ for which each $M_{\{i, k\}}$ is positive semi-definite. Thus, we do not have to search for the existence of such an $x_{\{i, k\}}$ numerically. To be precise, we will show that we can always choose

$$
x_{\{i, k\}}=\frac{1}{C_{i k}}
$$

to make each $M_{\{i, k\}}$ positive semi-definite. We note, however, that such a choice may not produce the best bounds (since the various bounds derived depend on $x_{\{i, k\}}$ ). Hence, in order to minimize the various bounds we may still want to numerically find alternative values for $x_{\{i, k\}}$, for which the matrices (A.2), (A.8) are also positive semi-definite. 
The correctness of the value (C.1) can be motivated physically as follows. Consider a system of two particles with masses $m=1$ with the Lorentz-invariant Lagrangian

$$
L^{i, k}=p^{i} \cdot p^{k}, \quad i \neq k,
$$

where $p^{i}$ for every $i, k=1, \ldots, N$ are the particle momenta. Due to space-time invariance, the respective Noether currents are stress-energy tensors,

$$
T_{\mu, \nu}^{i, k}=p_{\mu}^{i} p_{\nu}^{k}+p_{\mu}^{k} p_{\nu}^{i}-L^{i, k} \eta_{\mu \nu}, \quad i \neq k
$$

With the identification $\frac{1}{x^{i, k}} \equiv L^{i, k}$, these stress-energy tensors are precisely the matrices (A.8) multiplied by $\frac{1}{x^{i, k}}$. By the positive energy theorem in general relativity the $T_{\mu, \nu}^{i, k}$ are positive semi-definite for every chosen pair of time- or light-like $(n+1)$-vectors $p^{i}, p^{k}$.

In the following, we prove explicitly that the the matrices in (A.8), i.e. the stress energy tensors (C.3), are indeed positive semi-definite for time- or light-like $(n+1)$-vectors $p^{i}, p^{k}$. To this end, we will need the following general fact:

Lemma 7. For any $n \times n$ matrix $M$ and any invertible $n \times n$ matrix $A, M$ is positive semi-definite if and only if $A^{T} M A$ is positive semi-definite.

Using of lemma 7, we can prove positive semi-definiteness of $\tilde{M}_{\{i, k\}}$ by instead proving positive semi-definiteness of $A^{T} \tilde{M}_{\{i, k\}} A$, where $A$ is a suitably chosen invertible matrix so that $A^{T} \tilde{M}_{\{i, k\}} A$ takes a simpler form than $\tilde{M}_{\{i, k\}}$. We will discuss how to choose $A$ shortly. First, recall from table 3 that each Kähler cone generator $v_{i}$ is either time-like or light-like with Minkowski inner product $\eta\left(v_{i}, v_{i}\right)$ either 1 or 0 , and all the Kähler cone generators belong to the same light cone (the future-directed light cone). We choose $A$ as follows:

Case 1. Suppose $\tilde{M}_{\{i, k\}}$, defined in (A.8), has at least one of its $v_{i}, v_{k}$ with Minkowski inner product 1 . WLOG say $\eta\left(v_{i}, v_{i}\right)=1$. Then there is a matrix $A \in O(1, n)$ such that

$$
A^{T} v_{i}=(1,0, \ldots, 0)^{T} .
$$

We note that this is just a Lorentz transformation to the rest frame. Pick this matrix as the invertible matrix $A$ in lemma 7.

Case 2. Suppose $\tilde{M}_{\{i, k\}}$, defined in (A.8), has both of its $v_{i}, v_{k}$ with Minkowski inner product 0 . Then there exists a Lorentz transformation $A^{\prime} \in O(1, n)$ such that

$$
A^{\prime T} v_{i}=\left(a_{0}, a_{0}, 0, \ldots, 0\right)^{T}, \quad v_{k}^{T} A^{\prime}=\left(b_{0}, b_{1}, b_{2}, 0, \ldots, 0\right),
$$

where $a_{0}, b_{0}, b_{1}, b_{2} \in \mathbb{Q}$ and $b_{0}^{2}-b_{1}^{2}-b_{2}^{2}=0$. Pick $A^{\prime}$ as the invertible matrix in lemma 7 .

The above mentioned matrices in $O(1, n)$ exist because of the following general lemma:

Lemma 8. For any vector $v \in \mathbb{R}^{1, n}$ which Minkowski inner product $\eta(v, v)=1$, there exists a matrix $A \in O(1, n)$ such that $A^{T} v=(1,0, \ldots, 0)^{T}$. For any pair of vector $v_{i}, v_{k} \in \mathbb{R}^{1, n}$ both with Minkowski inner product $\eta\left(v_{i}, v_{i}\right)=\eta\left(v_{k}, v_{k}\right)=0$, there exists a matrix $A^{\prime} \in O(1, n)$ such that $A^{\prime T} v_{i}=\left(a_{0}, a_{0}, 0, \ldots, 0\right)^{T}, v_{k}^{T} A^{\prime}=\left(b_{0}, b_{1}, b_{2}, 0, \ldots, 0\right)$ where $a_{0}, b_{0}, b_{1}, b_{2} \in \mathbb{R}$ and $b_{0}^{2}-b_{1}^{2}-b_{2}^{2}=0$. 
Proof. First consider any $v \in \mathbb{R}^{1, n}$ with Minkowski inner product $\eta(v, v)=1$. Since $\eta(v, v)=1 \neq 0$, we can carry out the Gram-Schmidt process starting with $v$ as the first vector to generate an orthonormal basis $\left\{e_{1}=v, e_{2}, \ldots, e_{n+1}\right\}$ for $\mathbb{R}^{1, n}$. Define the $(1+n) \times(1+n)$ matrix $B$ whose $i$-th column is $e_{i}$, and define $A=\eta B$. Then $A^{T} v=$ $(1,0, \ldots, 0)^{T}$ by orthonormality. Both $B$ and $\eta$ are in $O(1, n)$ because each has its columns orthonormal to one another under the $(1, n)$ Minkowski metric. Thus $A=\eta B \in O(1, n)$.

Next consider any pair of vector $v_{i}, v_{k} \in \mathbb{R}^{1, n}$, both with Minkowski inner product $\eta\left(v_{i}, v_{i}\right)=\eta\left(v_{k}, v_{k}\right)=0$. If both are equal to the trivial vector $(0, \ldots, 0)^{T}$, let $A^{\prime}$ be any matrix in $O(1, n)$ and we are done with $a_{0}=b_{0}=b_{1}=b_{2}=0$. Thus assume at least one of them, WLOG say $v_{i}$, is not the trivial vector. Let $v_{i}=\left(a_{0}, \mathbf{a}\right)^{T}$ where $\mathbf{a}=\left(a_{1}, \ldots, a_{n}\right)^{T} \in \mathbb{R}^{n}$. Since $\eta\left(v_{i}, v_{i}\right)=0$ and $v_{i}$ is not the trivial vector, the Euclidean norm of $\mathbf{a},|\mathbf{a}|=a_{0} \neq 0$ ( $a_{0}$ is positive because $v_{i}$ is in the positive light cone). We can thus use $\mathbf{a} /|\mathbf{a}|$ as the first vector in the Gram-Schmidt process on $\mathbb{R}^{n}$ to generate an orthonormal basis $\left\{e_{1}=\mathbf{a} /|\mathbf{a}|, e_{2}, \ldots, e_{n}\right\}$ for $\mathbb{R}^{n}$. Define the $n \times n$ matrix $B^{\prime}$ whose $i$-th column is $e_{i}$. Then define the $(1+n) \times(1+n)$ block diagonal matrix $B^{\prime \prime}$ by

$$
B^{\prime \prime}=\left(\begin{array}{ll}
1 & 0 \\
0 & B^{\prime}
\end{array}\right) \text {. }
$$

$B^{\prime \prime} \in O(1, n)$ because its columns are orthonormal. Also $B^{\prime \prime} T v_{i}=\left(a_{0}, a_{0}, 0, \ldots, 0\right)^{T}$. Let $v_{k}^{T} B^{\prime \prime}=\left(b_{0}, b_{1}, \mathbf{b}^{\prime}\right)$ where $\mathbf{b}^{\prime}=\left(b_{2}^{\prime}, \ldots, b_{n}^{\prime}\right) \in \mathbb{R}^{n-1}$. If $\mathbf{b}^{\prime}$ is the trivial vector in $\mathbb{R}^{n-1}$, we are done by setting $A^{\prime}=B^{\prime \prime}$ and $b_{2}=0$. If $\mathbf{b}^{\prime}$ is not the trivial vector, we can again use $\mathbf{b}^{\prime} /\left|\mathbf{b}^{\prime}\right|$ as the first vector in the Gram-Schmidt process on $\mathbb{R}^{n-1}$ to generate an orthonormal basis $\left\{e_{1}=\mathbf{b}^{\prime} /\left|\mathbf{b}^{\prime}\right|, e_{2}, \ldots, e_{n-1}\right\}$ for $\mathbb{R}^{n-1}$. Define the $(n-1) \times(n-1)$ matrix $C^{\prime}$ whose $i$-th column is $e_{i}$. Then define the $(1+n) \times(1+n)$ block diagonal matrix $C^{\prime \prime}$ by

$$
C^{\prime \prime}=\left(\begin{array}{lll}
1 & 0 & 0 \\
0 & 1 & 0 \\
0 & 0 & C^{\prime}
\end{array}\right)
$$

$C^{\prime \prime} \in O(1, n)$ because its columns are orthonormal. Let $A^{\prime}=B^{\prime \prime} C^{\prime \prime} . A^{\prime} \in O(1, n)$ because $B^{\prime \prime}, C^{\prime \prime}$ are. We also have $A^{\prime T} v_{i}=\left(a_{0}, a_{0}, 0, \ldots, 0\right)^{T}, v_{k}^{T} A^{\prime}=\left(b_{0}, b_{1}, b_{2}, 0, \ldots, 0\right)$ where $b_{2}=\left|\mathbf{b}^{\prime}\right|$. Notice that $b_{0}^{2}-b_{1}^{2}-b_{2}^{2}=\eta\left(A^{\prime T} v_{k}, A^{\prime T} v_{k}\right)=\eta\left(v_{k}, v_{k}\right)=0$, where in the second equality we used the facts that $O(1, n)$ is closed under transposition, so $A^{\prime T} \in O(1, n)$, and that the Lorentz group $O(1, n)$ preserves $\eta(\cdot, \cdot)$.

Before justifying the choice $x_{\{i, k\}}=1 / C_{i k}$, we need to show $C_{i k} \neq 0$ for $i \neq k$ (by definition we always have $i \neq k$ in $x_{\{i, k\}}$ and $M_{\{i, k\}}$ ). Also recall that in (3.9), we require $x_{\{i, k\}} \in$ $\mathbb{Q}^{+}$. Thus a prerequisite for the choice $x_{\{i, k\}}=1 / C_{i k}$ to make sense is that $C_{i k}>0$ for $i \neq k$ $\left(C_{i k}\right.$ is already an integer since it is an intersection number). We have the following lemma:

Lemma 9. $C_{i k} \geq 0$. Furthermore, $C_{i k}>0$ if $i \neq k ; C_{i i}=0$ if and only if $v_{i}$ is lightlike; i.e. $\eta\left(v_{i}, v_{i}\right)=0$. 
Proof. Recall we have

$$
C_{i k}=\eta\left(v_{i}, v_{k}\right) .
$$

Also, by table 3 , all the Kähler cone generators $v_{i}, v_{k}$ are either time-like or light-like vectors belonging to the same light cone. In addition, of course neither of them is the trivial vector $\mathbf{0}$, because they generate the Kähler cone. This means all their inner products are nonnegative, i.e. $C_{i k}=\eta\left(v_{i}, v_{k}\right) \geq 0$, where equality $C_{i k}=\eta\left(v_{i}, v_{k}\right)=0$ holds only when $v_{i}$ and $v_{k}$ are parallel light-like vectors. This implies that $v_{i}$ and $v_{k}$ are not independent, so they must be the same Kähler cone generator $v_{i}=v_{k}$.

With this, we can prove the following theorem:

Theorem 5. Let $x_{\{i, k\}}=1 / C_{i k}$. Then $M_{\{i, k\}}$ is positive semi-definite.

Proof. It is equivalent to prove that with $x_{\{i, k\}}=1 / C_{i k}, A^{T} \tilde{M}_{\{i, k\}} A$ or $A^{\prime T} \tilde{M}_{\{i, k\}} A^{\prime}$, depending on which case above we are refering to is positive semi-definite, where $A$ (or $\left.A^{\prime}\right)$ is the appropriately chosen matrix in $O(1, n)$ discussed above.

Case 1. Suppose $\tilde{M}_{\{i, k\}}$, defined in (A.8), has at least one of its $v_{i}, v_{k}$ with Minkowski inner product 1 . WLOG say $\eta\left(v_{i}, v_{i}\right)=1$. Then

$$
\begin{aligned}
& A^{T} \tilde{M}_{\{i, k\}} A=x_{\{i, k\}} A^{T}\left(v_{i} \cdot v_{k}^{T}+v_{k} \cdot v_{i}^{T}\right) A-\eta \\
& =\frac{1}{c_{0}}\left((1,0, \ldots, 0)^{T}\left(c_{0}, c_{1}, \ldots, c_{n}\right)+\left(c_{0}, c_{1}, \ldots, c_{n}\right)^{T}(1,0, \ldots, 0)\right)-\eta \\
& =\left(\begin{array}{cccccc}
1 & \frac{c_{1}}{c_{0}} & \frac{c_{2}}{c_{0}} & \frac{c_{3}}{c_{0}} & \ldots & \frac{c_{n}}{c_{0}} \\
\frac{c_{1}}{c_{0}} & 1 & 0 & 0 & \ldots & 0 \\
\frac{c_{2}}{c_{0}} & 0 & 1 & 0 & \ldots & 0 \\
\cdot & \cdot & . & . & . & . \\
\frac{c_{n}}{c_{0}} & 0 & 0 & . & . & 1
\end{array}\right),
\end{aligned}
$$

where in the first equality, we used the fact that $A \in O(1, n)$ if and only if $A^{T} \eta A=\eta$. In the second equality, we used (C.4) and let $A^{T} v_{k}=\left(c_{0}, c_{1}, \ldots, c_{n}\right)^{T}$, so $C_{i k}=\eta\left(v_{i}, v_{k}\right)=$ $\eta\left(A^{T} v_{i}, A^{T} v_{k}\right)=\eta\left((1,0, \ldots, 0)^{T},\left(c_{0}, c_{1}, \ldots, c_{n}\right)^{T}\right)=c_{0}$ (notice that $O(1, n)$ is closed under transposition, so $A^{T} \in O(1, n)$ and thus $A^{T}$ preserves the inner product $\left.\eta(\cdot, \cdot)\right)$. It is not hard to see that the characteristic equation of $A^{T} \tilde{M}_{\{i, k\}} A$ is

$$
\operatorname{det}\left(A^{T} \tilde{M}_{\{i, k\}} A-\lambda I\right)=(1-\lambda)^{n-1}\left(\lambda^{2}-2 \lambda+1-\frac{1}{c_{0}^{2}} \sum_{j=1}^{n} c_{j}^{2}\right)=0,
$$

so the eigenvalues are

$$
\lambda=\{\underbrace{1, \ldots 1}_{n-1},\left(1 \pm \sqrt{\sum_{j=1}^{n} \frac{c_{j}^{2}}{c_{0}^{2}}}\right)\} .
$$


Since

$$
0 \leq 0 \text { or } 1=\eta\left(v_{k}, v_{k}\right)=\eta\left(A^{T} v_{k}, A^{T} v_{k}\right)=\eta\left(\left(c_{0}, c_{1}, \ldots, c_{n}\right)^{T},\left(c_{0}, c_{1}, \ldots, c_{n}\right)^{T}\right)=c_{0}^{2}-\sum_{j=1}^{n} c_{j}^{2},
$$

we must have

$$
1 \geq \sqrt{\sum_{j=1}^{n} \frac{c_{j}^{2}}{c_{0}^{2}}}
$$

so all the eigenvalues in (C.11) are non-negative. In particular, if $\eta\left(v_{k}, v_{k}\right)=c_{0}^{2}-\sum_{j=1}^{n} c_{j}^{2}=$ 1 , the eigenvalues will be

$$
\lambda=\{\underbrace{1, \ldots 1}_{n-1},\left(1 \pm \sqrt{1-\frac{1}{c_{0}^{2}}}\right)\} .
$$

If $\eta\left(v_{k}, v_{k}\right)=c_{0}^{2}-\sum_{j=1}^{n} c_{j}^{2}=0$, the eigenvalues will be

$$
\lambda=\{\underbrace{1, \ldots 1}_{n-1}, 0,2\} .
$$

Case 2. Suppose $\tilde{M}_{\{i, k\}}$, defined in (A.8), has both of its $v_{i}, v_{k}$ with Minkowski inner product 0 . Then

$$
\begin{aligned}
& A^{\prime T} \tilde{M}_{\{i, k\}} A^{\prime}=x_{\{i, k\}} A^{\prime T}\left(v_{i} \cdot v_{k}^{T}+v_{k} \cdot v_{i}^{T}\right) A^{\prime}-\eta \\
& =\frac{1}{b_{0}-b_{1}}\left((1,1,0, \ldots, 0)^{T}\left(b_{0}, b_{1}, b_{2}, 0 \ldots, 0\right)+\left(b_{0}, b_{1}, b_{2}, 0, \ldots, 0\right)^{T}(1,1,0, \ldots, 0)\right)-\eta \\
& =\left(\begin{array}{cccccc}
\frac{b_{0}+b_{1}}{b_{0}-b_{1}} & \frac{b_{0}+b_{1}}{b_{0}-b_{1}} & \frac{b_{2}}{b_{0}-b_{1}} & 0 & \ldots & 0 \\
\frac{b_{0}+b_{1}}{b_{0}-b_{1}} & \frac{b_{0}+b_{1}}{b_{0}-b_{1}} & \frac{b_{2}}{b_{0}-b_{1}} & 0 & \ldots & 0 \\
\frac{b_{2}}{b_{0}-b_{1}} & \frac{b_{2}}{b_{0}-b_{1}} & 1 & 0 & \ldots & 0 \\
0 & \cdot & 0 & 1 & \cdot & \cdot \\
\cdot & \cdot & \cdot & \cdot & \cdot & \cdot \\
0 & . & 0 & \cdot & \cdot & 1
\end{array}\right),
\end{aligned}
$$

where in the second equality we used (C.5) and $C_{i k}=\eta\left(v_{i}, v_{k}\right)=\eta\left(A^{\prime T} v_{i}, A^{\prime T} v_{k}\right)=$ $\eta\left(\left(a_{0}, a_{0}, 0, \ldots, 0\right)^{T},\left(b_{0}, b_{1}, b_{2}, 0, \ldots, 0\right)^{T}\right)=a_{0}\left(b_{0}-b_{1}\right)$. Letting

$$
s \equiv \frac{b_{0}+b_{1}}{b_{0}-b_{1}} \quad \sqrt{s}=\frac{b_{2}}{b_{0}-b_{1}},
$$

where in the second equation we used the relationship $b_{0}^{2}-b_{1}^{2}-b_{2}^{2}=0$, it is not hard to see that the characteristic equation of $A^{\prime T} \tilde{M}_{\{i, k\}} A^{\prime}$ is

$$
\operatorname{det}\left(A^{\prime} T \tilde{M}_{\{i, k\}} A^{\prime}-\lambda I\right)=(1-\lambda)^{n-2} \lambda^{2}(2 s+1-\lambda)=0,
$$


so the eigenvalues are

$$
\lambda=\{\underbrace{1, \ldots 1}_{n-2}, 0,0, \frac{3 b_{0}+b_{1}}{b_{0}-b_{1}}\} .
$$

The last eigenvalue $\frac{3 b_{0}+b_{1}}{b_{0}-b_{1}}$ is positive because $b_{0}^{2}-b_{1}^{2}-b_{2}^{2}=0$, so $\left|b_{0}\right|>\left|b_{1}\right|$.

Notice that the only required condition for this general proof is that all the Kähler cone generators $v_{i}, v_{k}$ are either time-like or light-like, and belong to the same light cone. This light cone does not need to be the positive one. Indeed, it is not hard to see that if all the Kähler cone generators were to belong to the negative light cone, the proof still holds with slight modifications at the relevant parts. Also, the time-like Kähler cone generators can always be rescaled to have Minkowski inner product $\eta\left(v_{i}, v_{i}\right)=1$. In summary, we have the following corollary:

Corollary 6. If all the Kähler cone generators $v_{i}, v_{k}$ are either time-like or light-like, and belong to the same light cone, then each matrix $M_{\{i, k\}}$ will be positive semi-definite by setting $x_{\{i, k\}}=1 / C_{i k}$.

Open Access. This article is distributed under the terms of the Creative Commons Attribution License (CC-BY 4.0), which permits any use, distribution and reproduction in any medium, provided the original author(s) and source are credited.

\section{References}

[1] S. Kachru, R. Kallosh, A.D. Linde and S.P. Trivedi, De Sitter vacua in string theory, Phys. Rev. D 68 (2003) 046005 [hep-th/0301240] [INSPIRE].

[2] V. Balasubramanian, P. Berglund, J.P. Conlon and F. Quevedo, Systematics of moduli stabilisation in Calabi-Yau flux compactifications, JHEP 03 (2005) 007 [hep-th/0502058] [INSPIRE].

[3] J. Louis, M. Rummel, R. Valandro and A. Westphal, Building an explicit de Sitter, JHEP 10 (2012) 163 [arXiv: 1208.3208] [INSPIRE].

[4] D. Martinez-Pedrera, D. Mehta, M. Rummel and A. Westphal, Finding all flux vacua in an explicit example, JHEP 06 (2013) 110 [arXiv:1212.4530] [INSPIRE].

[5] M. Cicoli et al., Explicit de Sitter flux vacua for global string models with chiral matter, JHEP 05 (2014) 001 [arXiv: 1312.0014] [INSPIRE].

[6] R. Bousso and J. Polchinski, Quantization of four form fluxes and dynamical neutralization of the cosmological constant, JHEP 06 (2000) 006 [hep-th/0004134] [INSPIRE].

[7] F. Denef and M.R. Douglas, Computational complexity of the landscape. I, Annals Phys. 322 (2007) 1096 [hep-th/0602072] [INSPIRE].

[8] M. Cvetič, I. Garcia-Etxebarria and J. Halverson, On the computation of non-perturbative effective potentials in the string theory landscape: IIB/F-theory perspective, Fortsch. Phys. 59 (2011) 243 [arXiv:1009.5386] [INSPIRE].

[9] F. Denef and M.R. Douglas, Distributions of flux vacua, JHEP 05 (2004) 072 [hep-th/0404116] [INSPIRE]. 
[10] F. Denef and M.R. Douglas, Distributions of nonsupersymmetric flux vacua, JHEP 03 (2005) 061 [hep-th/0411183] [INSPIRE].

[11] R. Donagi and M. Wijnholt, Model building with F-theory, Adv. Theor. Math. Phys. 15 (2011) 1237 [arXiv:0802.2969] [InSPIRE].

[12] C. Beasley, J.J. Heckman and C. Vafa, GUTs and exceptional branes in F-theory - I, JHEP 01 (2009) 058 [arXiv:0802.3391] [INSPIRE].

[13] C. Vafa, The string landscape and the swampland, hep-th/0509212 [INSPIRE].

[14] A.M. Uranga, D-brane probes, RR tadpole cancellation and K-theory charge, Nucl. Phys. B 598 (2001) 225 [hep-th/0011048] [INSPIRE].

[15] G. Aldazabal, S. Franco, L.E. Ibáñez, R. Rabadán and A.M. Uranga, $D=4$ chiral string compactifications from intersecting branes, J. Math. Phys. 42 (2001) 3103 [hep-th/0011073] [INSPIRE].

[16] L.E. Ibáñez, F. Marchesano and R. Rabadán, Getting just the Standard Model at intersecting branes, JHEP 11 (2001) 002 [hep-th/0105155] [INSPIRE].

[17] M. Cvetič, G. Shiu and A.M. Uranga, Chiral four-dimensional $N=1$ supersymmetric type IIA orientifolds from intersecting D6 branes, Nucl. Phys. B 615 (2001) 3 [hep-th/0107166] [INSPIRE].

[18] J. Halverson, Anomaly nucleation constrains SU(2) gauge theories, Phys. Rev. Lett. 111 (2013) 261601 [arXiv:1310.1091] [INSPIRE].

[19] M. Cvetič, T.W. Grimm and D. Klevers, Anomaly cancellation and Abelian gauge symmetries in F-theory, JHEP 02 (2013) 101 [arXiv:1210.6034] [INSPIRE].

[20] W. Lerche, D. Lüst and A.N. Schellekens, Chiral four-dimensional heterotic strings from selfdual lattices, Nucl. Phys. B 287 (1987) 477 [INSPIRE].

[21] F. Gmeiner, R. Blumenhagen, G. Honecker, D. Lüst and T. Weigand, One in a billion: MSSM-like D-brane statistics, JHEP 01 (2006) 004 [hep-th/0510170] [INSPIRE].

[22] S.H. Katz and C. Vafa, Matter from geometry, Nucl. Phys. B 497 (1997) 146 [hep-th/9606086] [INSPIRE].

[23] O. DeWolfe and B. Zwiebach, String junctions for arbitrary Lie algebra representations, Nucl. Phys. B 541 (1999) 509 [hep-th/9804210] [INSPIRE].

[24] A. Grassi and D.R. Morrison, Group representations and the Euler characteristic of elliptically fibered Calabi-Yau threefolds, math.AG/0005196 [INSPIRE].

[25] A. Grassi and D.R. Morrison, Anomalies and the Euler characteristic of elliptic Calabi-Yau threefolds, Commun. Num. Theor. Phys. 6 (2012) 51 [arXiv:1109.0042] [inSPIRE].

[26] D.R. Morrison and W. Taylor, Matter and singularities, JHEP 01 (2012) 022 [arXiv:1106.3563] [INSPIRE].

[27] A. Grassi, J. Halverson and J.L. Shaneson, Matter from geometry without resolution, JHEP 10 (2013) 205 [arXiv:1306.1832] [INSPIRE].

[28] H. Hayashi, C. Lawrie, D.R. Morrison and S. Schäfer-Nameki, Box graphs and singular fibers, JHEP 05 (2014) 048 [arXiv: 1402 .2653] [INSPIRE].

[29] A. Grassi, J. Halverson and J.L. Shaneson, Non-Abelian gauge symmetry and the Higgs mechanism in F-theory, arXiv:1402.5962 [INSPIRE]. 
[30] M. Esole, S.-H. Shao and S.-T. Yau, Singularities and gauge theory phases, arXiv: 1402.6331 [INSPIRE].

[31] D.R. Morrison and D.S. Park, F-theory and the Mordell-Weil group of elliptically-fibered Calabi-Yau threefolds, JHEP 10 (2012) 128 [arXiv:1208.2695] [INSPIRE].

[32] J. Borchmann, C. Mayrhofer, E. Palti and T. Weigand, Elliptic fibrations for $\mathrm{SU}(5) \times \mathrm{U}(1) \times \mathrm{U}(1)$ F-theory vacua, Phys. Rev. D 88 (2013) 046005 [arXiv: 1303.5054] [INSPIRE].

[33] M. Cvetič, D. Klevers and H. Piragua, F-theory compactifications with multiple U(1)-factors: constructing elliptic fibrations with rational sections, JHEP 06 (2013) 067 [arXiv: 1303.6970] [INSPIRE].

[34] T.W. Grimm, A. Kapfer and J. Keitel, Effective action of $6 D$ F-theory with U(1) factors: rational sections make Chern-Simons terms jump, JHEP 07 (2013) 115 [arXiv:1305.1929] [INSPIRE].

[35] V. Braun, T.W. Grimm and J. Keitel, Geometric engineering in toric F-theory and GUTs with U(1) gauge factors, JHEP 12 (2013) 069 [arXiv:1306.0577] [INSPIRE].

[36] M. Cvetič, A. Grassi, D. Klevers and H. Piragua, Chiral four-dimensional F-theory compactifications with $\mathrm{SU}(5)$ and multiple $\mathrm{U}(1)$-factors, JHEP 04 (2014) 010 [arXiv: 1306.3987] [INSPIRE].

[37] J. Borchmann, C. Mayrhofer, E. Palti and T. Weigand, SU(5) tops with multiple U(1)s in F-theory, Nucl. Phys. B $\mathbf{8 8 2}$ (2014) 1 [arXiv:1307.2902] [INSPIRE].

[38] M. Cvetič, D. Klevers and H. Piragua, F-theory compactifications with multiple U(1)-factors: addendum, JHEP 12 (2013) 056 [arXiv: 1307.6425] [INSPIRE].

[39] M. Cvetič, D. Klevers, H. Piragua and P. Song, Elliptic fibrations with rank three Mordell-Weil group: F-theory with $\mathrm{U}(1) \times \mathrm{U}(1) \times \mathrm{U}(1)$ gauge symmetry, JHEP 03 (2014) 021 [arXiv: 1310.0463] [INSPIRE].

[40] M.R. Douglas and W. Taylor, The landscape of intersecting brane models, JHEP 01 (2007) 031 [hep-th/0606109] [INSPIRE].

[41] R. Blumenhagen, M. Cvetič, P. Langacker and G. Shiu, Toward realistic intersecting D-brane models, Ann. Rev. Nucl. Part. Sci. 55 (2005) 71 [hep-th/0502005] [InSPIRE].

[42] R. Blumenhagen, B. Körs, D. Lüst and S. Stieberger, Four-dimensional string compactifications with D-branes, orientifolds and fluxes, Phys. Rept. 445 (2007) 1 [hep-th/0610327] [INSPIRE].

[43] M. Cvetič and J. Halverson, TASI lectures: particle physics from perturbative and non-perturbative effects in D-braneworlds, arXiv:1101.2907 [INSPIRE].

[44] R. Blumenhagen, F. Gmeiner, G. Honecker, D. Lüst and T. Weigand, The statistics of supersymmetric D-brane models, Nucl. Phys. B 713 (2005) 83 [hep-th/0411173] [INSPIRE].

[45] M. Cvetič, G. Shiu and A.M. Uranga, Three family supersymmetric standard-like models from intersecting brane worlds, Phys. Rev. Lett. 87 (2001) 201801 [hep-th/0107143] [INSPIRE].

[46] M. Cvetič, T. Li and T. Liu, Supersymmetric Pati-Salam models from intersecting D6-branes: a road to the Standard Model, Nucl. Phys. B 698 (2004) 163 [hep-th/0403061] [InSPIRE].

[47] L.B. Anderson and W. Taylor, Geometric constraints in dual F-theory and heterotic string compactifications, to appear. 
[48] R. Blumenhagen, G. Honecker and T. Weigand, Supersymmetric (non-)Abelian bundles in the type I and $\mathrm{SO}(32)$ heterotic string, JHEP 08 (2005) 009 [hep-th/0507041] [INSPIRE].

[49] C. Bachas, A way to break supersymmetry, hep-th/9503030 [INSPIRE].

[50] D. Honda and T. Okuda, Exact results for boundaries and domain walls in $2 d$ supersymmetric theories, arXiv:1308.2217 [INSPIRE].

[51] S. Sugishita and S. Terashima, Exact results in supersymmetric field theories on manifolds with boundaries, JHEP 11 (2013) 021 [arXiv:1308.1973] [INSPIRE].

[52] K. Hori and M. Romo, Exact results in two-dimensional $(2,2)$ supersymmetric gauge theories with boundary, arXiv:1308.2438 [INSPIRE].

[53] J. Halverson, H. Jockers, J.M. Lapan and D.R. Morrison, Perturbative corrections to Kähler moduli spaces, arXiv: 1308.2157 [INSPIRE].

[54] R. Friedman, J. Morgan and E. Witten, Vector bundles and F-theory, Commun. Math. Phys. 187 (1997) 679 [hep-th/9701162] [INSPIRE].

[55] D.R. Morrison and C. Vafa, Compactifications of F-theory on Calabi-Yau threefolds. 2, Nucl. Phys. B 476 (1996) 437 [hep-th/9603161] [INSPIRE].

[56] M.-X. Huang, A. Klemm and M. Poretschkin, Refined stable pair invariants for E-, $M$ - and [p,q]-strings, JHEP 11 (2013) 112 [arXiv:1308.0619] [INSPIRE].

[57] M.-X. Huang, A. Klemm, J. Reuter and M. Schiereck, Quantum geometry of del Pezzo surfaces in the Nekrasov-Shatashvili limit, arXiv:1401.4723 [INSPIRE].

[58] M. Demazure, H.C. Pinkham and B. Teissier, Séminaire sur les singularités des surfaces (in French), Lect. Notes Math. 777, Springer Verlag, Berlin Germany (1980).

[59] R. Donagi, Y.-H. He, B.A. Ovrut and R. Reinbacher, The particle spectrum of heterotic compactifications, JHEP 12 (2004) 054 [hep-th/0405014] [INSPIRE].

[60] A. Klemm, J. Manschot and T. Wotschke, Quantum geometry of elliptic Calabi-Yau manifolds, arXiv: 1205.1795 [INSPIRE].

[61] Sage Development Team collaboration, W. Stein et al., Sage mathematics software (version 6.0), http://www.sagemath.org/, (2013).

[62] F. Gmeiner and G. Honecker, Mapping an island in the landscape, JHEP 09 (2007) 128 [arXiv:0708.2285] [INSPIRE].

[63] G. Honecker, M. Ripka and W. Staessens, The importance of being rigid: D6-brane model building on $T^{6} / Z_{2} \times Z_{6}^{\prime}$ with discrete torsion, Nucl. Phys. B 868 (2013) 156 [arXiv: 1209.3010] [INSPIRE].

[64] T.W. Grimm, D. Klevers and M. Poretschkin, Fluxes and warping for gauge couplings in F-theory, JHEP 01 (2013) 023 [arXiv: 1202.0285] [INSPIRE].

[65] I. Garcia-Etxebarria, H. Hayashi, R. Savelli and G. Shiu, On quantum corrected Kähler potentials in F-theory, JHEP 03 (2013) 005 [arXiv: 1212.4831] [INSPIRE].

[66] T.W. Grimm, R. Savelli and M. Weissenbacher, On $\alpha^{\prime}$-corrections in $N=1$ F-theory compactifications, Phys. Lett. B 725 (2013) 431 [arXiv:1303.3317] [INSPIRE].

[67] T.W. Grimm, J. Keitel, R. Savelli and M. Weissenbacher, From M-theory higher curvature terms to $\alpha^{\prime}$-corrections in F-theory, arXiv:1312.1376 [INSPIRE]. 